University of Tennessee Health Science Center

UTHSC Digital Commons

\title{
Identification of Novel CYP2E1 Inhibitor to Investigate Cellular and Exosomal CYP2E1-Mediated Toxicity
}

\author{
Mohammad Arifur Rahman \\ University of Tennessee Health Science Center
}

Follow this and additional works at: https://dc.uthsc.edu/dissertations

Part of the Pharmacy and Pharmaceutical Sciences Commons

\footnotetext{
Recommended Citation

Rahman, Mohammad Arifur (0000-0002-5589-0114), "Identification of Novel CYP2E1 Inhibitor to Investigate Cellular and Exosomal CYP2E1-Mediated Toxicity" (2019). Theses and Dissertations (ETD). Paper 482. http://dx.doi.org/10.21007/etd.cghs.2019.0474.
}

This Dissertation is brought to you for free and open access by the College of Graduate Health Sciences at UTHSC Digital Commons. It has been accepted for inclusion in Theses and Dissertations (ETD) by an authorized administrator of UTHSC Digital Commons. For more information, please contact jwelch30@uthsc.edu. 


\title{
Identification of Novel CYP2E1 Inhibitor to Investigate Cellular and Exosomal CYP2E1-Mediated Toxicity
}

\author{
Abstract \\ acetaminophen- (APAP) induced toxicity and disease (e.g. HIV-1) pathogenesis. DAS imparts its \\ beneficial effect by inhibiting CYP2E1-mediated metabolism of xenobiotics, especially at high \\ research tool or as potential adjuvant therapy in CYP2E1-mediated pathologies. \\ Document Type \\ Dissertation \\ Degree Name \\ Doctor of Philosophy $(\mathrm{PhD})$ \\ Program \\ Pharmaceutical Sciences \\ Research Advisor \\ Santosh Kumar, Ph.D. \\ Keywords \\ Acetaminophen, Alcohol, CYP2E1, Diallyl Sulfide, Plasma exosomes, Toxicity \\ Subject Categories \\ Medicine and Health Sciences | Pharmacy and Pharmaceutical Sciences
}

Cytochrome P450 2E1 (CYP2E1)-mediated hepatic and extra-hepatic toxicity is of significant clinical importance. Diallyl sulfide (DAS) has been shown to prevent xenobiotics such as alcohol- (ALC/ETH), concentration. However, DAS also causes toxicity at relatively high dosages and with long exposure times. The objective of the first project was to find potent DAS analogs which can replace DAS as a 
Identification of Novel CYP2E1 Inhibitor to Investigate Cellular and Exosomal CYP2E1-Mediated Toxicity

\author{
A Dissertation \\ Presented for \\ The Graduate Studies Council \\ The University of Tennessee \\ Health Science Center \\ In Partial Fulfillment \\ Of the Requirements for the Degree \\ Doctor of Philosophy \\ From The University of Tennessee
}

By

Mohammad Arifur Rahman

June 2019 
Portions of chapter 1 (C) 2019 by Springer Nature. All other material @ 2019 by Mohammad Arifur Rahman. All rights reserved. 


\section{DEDICATION}

To my beloved parents- Mohammad Ishaque Ali and Ms. Hosne Ara (Baby). 


\section{ACKNOWLEDGEMENTS}

I would like to express my sincere gratitude to my mentor, Dr. Santosh Kumar for his guidance and support to make this project a success. He has not only helped me to develop as a scientist but also, most importantly, has guided me to become a better human being through his moral teachings. I am forever grateful to him for his outstanding mentorship throughout the years.

I am also thankful to my committee members, Dr. Bernd Meibohm, Dr. Wei Li, Dr. Ryan Yates, and Dr. CV Dash for their time, valuable inputs and suggestions to complete my project and dissertation. I would also like to acknowledge Springer US, Nature Publishing group, Elsevier Inc., John Wiley and Sons, and Elsevier B.V. for allowing me to reprint and modify the contents from the respective manuscripts into my dissertation.

My sincere appreciation to department of Pharmaceutical Sciences for the administrative and financial support to conduct my graduate study and project. I would also like to thank National Institutes of Health for providing grants: R01: AA022063 and R21: DA042374 to fund my project.

I would like to sincerely thank my wonderful lab mates, Narasimha Midde, Benjamin Patters, Namita Sinha, Yuqing Gong, Sunitha Kodidela, and PSS Rao for their help and support throughout this journey. I also want to recognize my friends in the department of Pharmaceutical Sciences, especially Ashit Trivedi, Andy Nishimoto, Bivash Mandal, and Chetan Rathi for their valuable time and friendship.

Last, but not the least, I would like to thank my beautiful wife, Sanjana Haque (Arthi) for supporting me in every possible way in this journey. She has been my best friend ever since she came into my life and helped me get through the tough moments with a smile in her face. I am eternally grateful to my beloved parents for believing me in the pursuit of my dreams. I am thankful to my mother-in-law, Mrs. Shaheda Khatun for putting her trust in me. I am blessed to have my sisters, Nilufar Yasmin and Shayonti Haque Ahona in my life. I express my deep gratitude towards my relatives, teachers, and all the well-wishers that have made my life worth living for. I feel extremely privileged to live on this beautiful planet, to be a one-of-a-kind speck in a tiny corner of the universe. 


\begin{abstract}
Cytochrome P450 2E1 (CYP2E1)-mediated hepatic and extra-hepatic toxicity is of significant clinical importance. Diallyl sulfide (DAS) has been shown to prevent xenobiotics such as alcohol- (ALC/ETH), acetaminophen- (APAP) induced toxicity and disease (e.g. HIV-1) pathogenesis. DAS imparts its beneficial effect by inhibiting CYP2E1-mediated metabolism of xenobiotics, especially at high concentration. However, DAS also causes toxicity at relatively high dosages and with long exposure times. The objective of the first project was to find potent DAS analogs which can replace DAS as a research tool or as potential adjuvant therapy in CYP2E1-mediated pathologies.

At first, we selected seven commercially available compounds that are similar to DAS (DAS analogs) based on computational docking study. We performed ligandCYP2E1 docking study to determine the binding mode and binding energy. The analysis suggested a relative potential for these DAS analogs as CYP2E1 inhibitor. We then performed comprehensive inhibition kinetics of DAS analogs and determined the relative IC50, Ki, and types of inhibition compared to that of DAS. The results showed that compared to DAS, diallyl ether (DE) and allyl methyl sulfide (AMS) have lower Ki values (3.1 and $4.4 \mu \mathrm{M}$, respectively vs. $6.3 \mu \mathrm{M}$ for DAS) and IC50 values (6.3 and 11.4 $\mu \mathrm{M}$, respectively vs $17.3 \mu \mathrm{M}$ for DAS). However, thiophene (TP) showed similar inhibitory capacities to that of DAS, and four other DAS analogs showed lower potency than DAS. From this project, we found relatively more potent inhibitors of CYP2E1 which can potentially replace DAS.
\end{abstract}

Next, we investigated these analogs of DAS for their improved toxicity profiles and their effectiveness in reducing ALC- and APAP-induced toxicity, and HIV-1 replication. In this project, we evaluated the toxicity and efficacy of these analogs using hepatocytes, monocytes, and astrocytes where CYP2E1 plays an important role in xenobiotic-mediated toxicity. Our results showed that thiophene, allyl methyl sulfide, diallyl ether, and 2-prop-2-enoxyacetamide are significantly less cytotoxic than DAS in these cells. Moreover, these analogs reduced ALC- and APAP-induced toxicity in hepatocytes and HIV-1 replication in monocytes more effectively than DAS. Overall, our findings are significant in terms of using these DAS analogs as a tool in vitro and in vivo, especially to examine chronic xenobiotic-induced toxicity and disease pathogenesis that occurs through the CYP2E1 pathway.

We also investigated the role of plasma exosomal CYP2E1 in mediating ALCand APAP-induced toxicity in hepatic and monocytic cells. Cellular CYP2E1 is wellknown to mediate ALC- and APAP-induced toxicity in hepatic and extra-hepatic cells. Although exosomes have been gaining importance in understanding the mechanism of intra- and inter-cellular communication, the functional role of drug metabolizing CYP enzymes in human plasma exosomes are yet to be explored. We recently reported that human plasma-derived exosomes contain substantial level of functional CYP2E1. In this project, we investigated the potential role of plasma exosomal CYP2E1 in mediating 
ALC and APAP induced toxicity. We treated hepatic and extra-hepatic (monocytic) cells with exosomes \pm ALC/APAP. We observed that the plasma exosomes containing CYP2E1 cargo further exacerbated ALC- and APAP-induced toxicity in both hepatic and monocytic cells. Further, both exosomes- and ALC/APAP-induced toxicity was reduced/abolished by CYP2E1 inhibitor, DE. However, only ALC-, but not exosomeinduced toxicity was reduced/abolished by CYP2E1 siRNA. These findings suggest that ALC/APAP-induced toxicity in the presence of exosomes are mediated, at least in part, by CYP2E1 enzyme. To validate these in vitro findings, we characterized plasma exosomal contents in a binge-drinking animal model and their effect on ALC/APAPinduced toxicity in monocytic cells. Our results showed that ALC exposure caused a significant induction of the plasma exosomal CYP2E1 level in a binge drinking murine model. These exosomes containing increased levels of CYP2E1 caused significant toxicity in monocytic cells compared to exosomes derived from control mice. Overall, our results showed an important role of exosomal CYP2E1 in exacerbating ALC- and APAP-induced toxicity. The study is significant in terms of understanding the role of exosomal CYP2E1 in cell-cell interactions, and their effects on drug-induced toxicity.

In summary, we have identified several superior inhibitors of CYP2E1 which can replace DAS as research tool and we have demonstrated for the first time that exosomal CYP2E1 may contribute to ALC- and APAP-induced cytotoxicity. 


\section{TABLE OF CONTENTS}

CHAPTER 1. INTRODUCTION .........................................................................................1

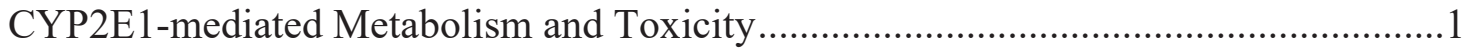

Epidemiology of ALC- and APAP-related disorders ............................................ 1

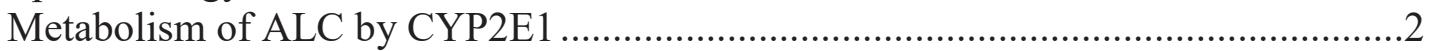

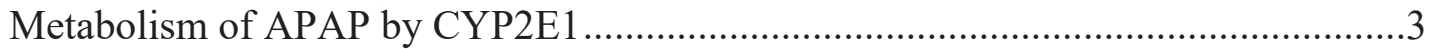

Involvement of CYP2E1 in the Toxicity of Hepatic and Extra-hepatic Cells................4

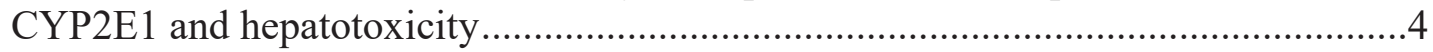

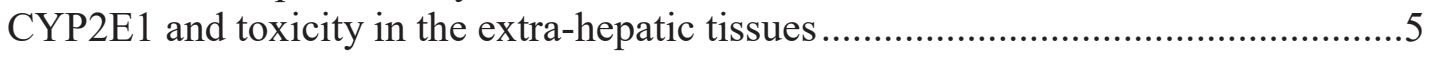

CYP2E1 and neurotoxicity .................................................................... 5

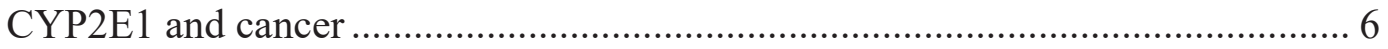

Involvement of Oxidative Stress and CYP2E1 in HIV-1 Pathogenesis .........................6

Potential of Diallyl Sulfide as a CYP2E1 Inhibitor ..................................................

Protective Effects of Diallyl Sulfide (DAS) ...........................................................

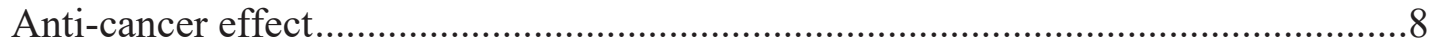

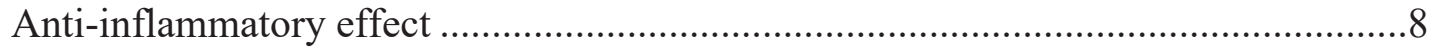

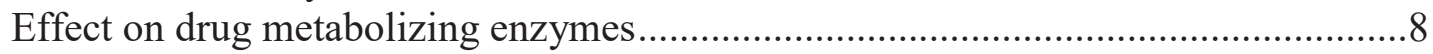

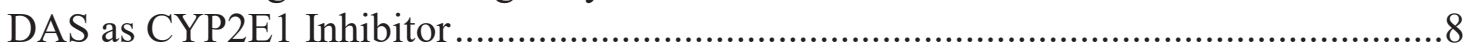

Protection against ALC-induced toxicity........................................................ 9

Protection against APAP- and other drug-induced toxicity ..................................9

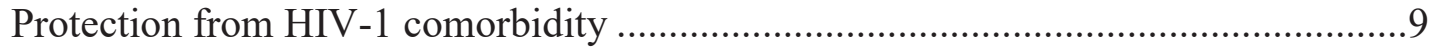

Metabolism of DAS by CYP2E1 and Potential Toxicity ..................................... 10

Exosomes as Intercellular Mediators in Alcohol-induced Pathologies .........................10

Alcohol-induced EVs/exosomes from Hepatic Cells in Cell-cell Communication........13

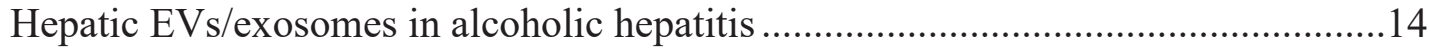

Hepatic EVs/exosomes in alcoholic liver disease...............................................14

Hepatic EVs/exosomes in fibrogenesis............................................................. 15

Alcohol and EVs/exosomes Derived from Extra-hepatic Tissues in Cell-cell

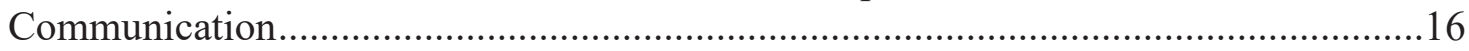

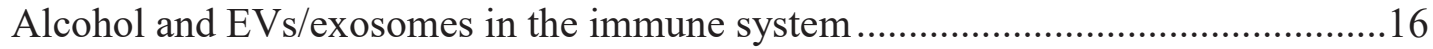

Alcohol and EVs/exosomes in epithelia and endothelia .......................................... 17

Alcohol and EVs/exosomes in the heart ........................................................... 18

Alcohol and EVs/exosomes in the pancreas ....................................................... 18

Plasma exosomes and cytochrome P450 enzymes ............................................ 18

Potential Mechanisms of Alcohol-induced Toxicity via EVs/exosomes......................19

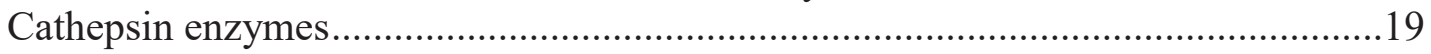

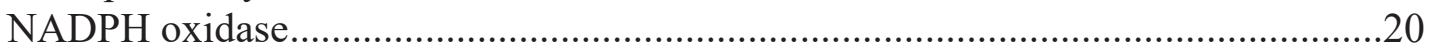

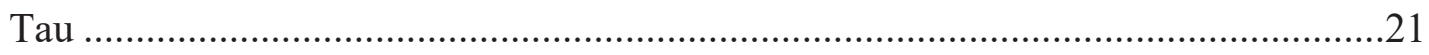

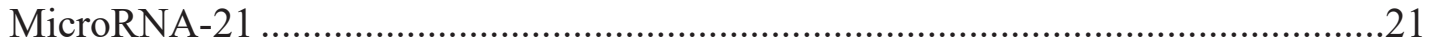

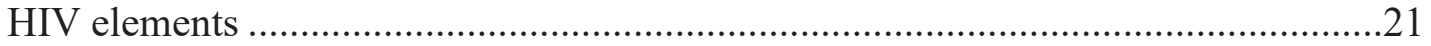

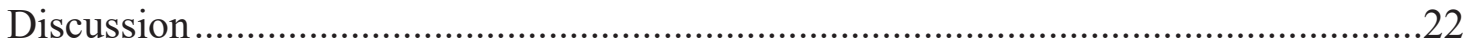

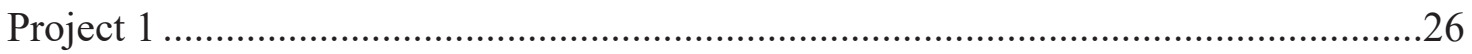

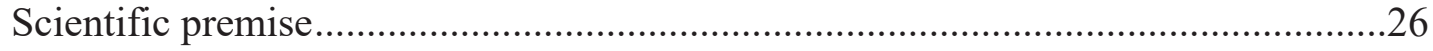

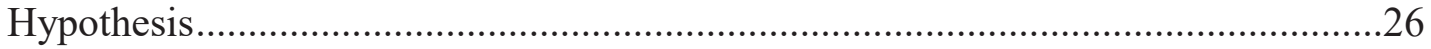




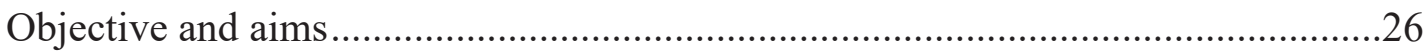

Aim 1: Kinetic characterization of DAS analogs for their novel role as .................

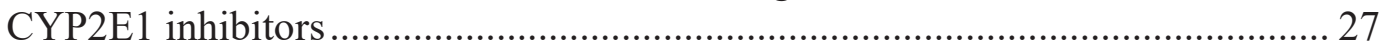

Aim 2: Evaluation of structural analogs of DAS as novel CYP2E1 inhibitors ........

for their protective effect against xenobiotic-induced toxicity and HIV ..................

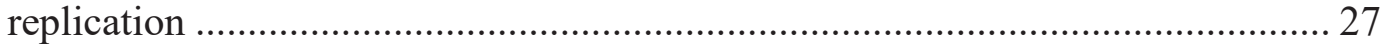

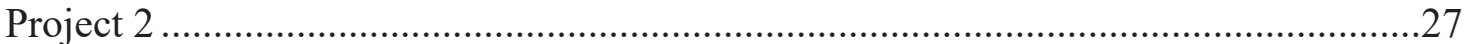

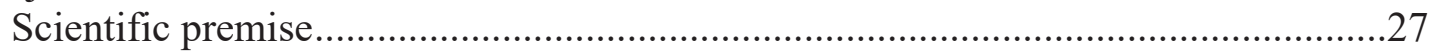

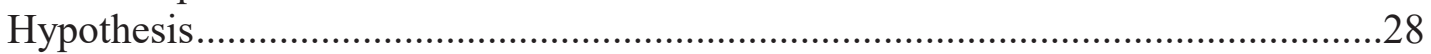

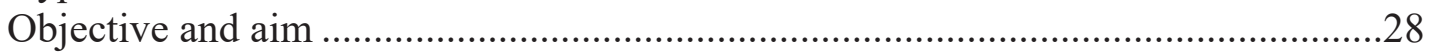

Aim: Investigation of the role of plasma exosomal CYP2E1 in alcohol- and

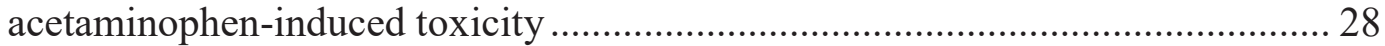

\section{CHAPTER 2. KINETIC CHARACTERIZATIONS OF DIALLYL SULFIDE ANALOGS FOR THEIR NOVEL ROLE AS CYP2E1 ENZYME INHIBITORS ...29}

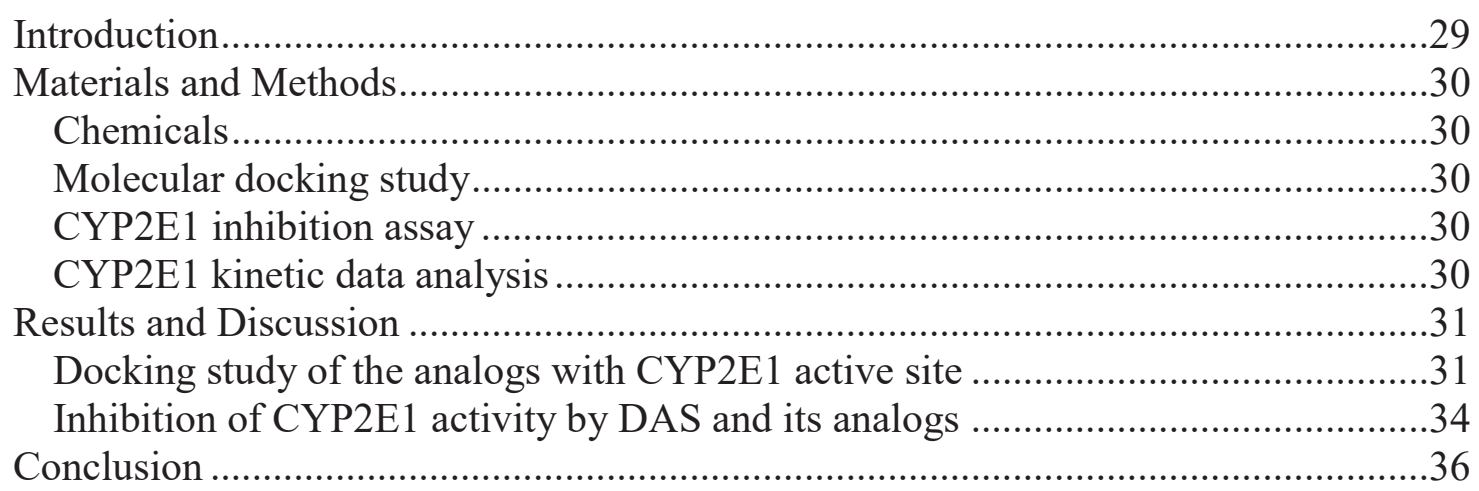

CHAPTER 3. IN VITRO EVALUATION OF STRUCTURAL ANALOGS OF DIALLYL SULFIDE AS NOVEL CYP2E1 INHIBITORS FOR THEIR PROTECTIVE EFFECT AGAINST XENOBIOTIC-INDUCED TOXICITY AND HIV REPLICATION ................................................................................38

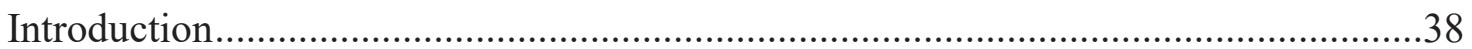

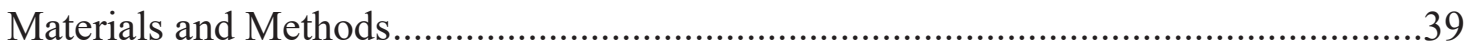

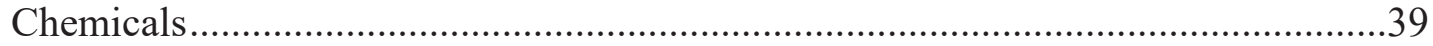

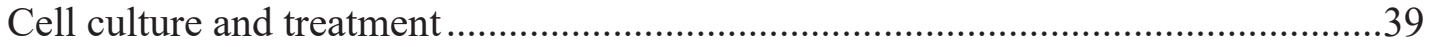

Determination of CYP2E1 activity in U937 monocytic and SVGA astrocytic

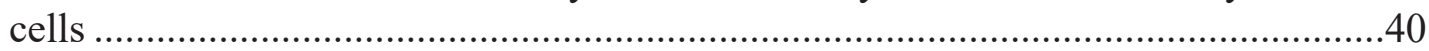

Measurement of reactive oxygen species (ROS) and cell viability using flow

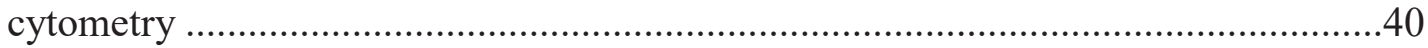

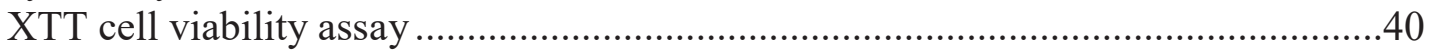

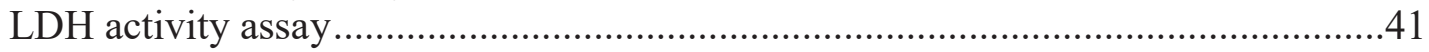

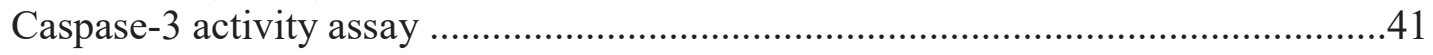

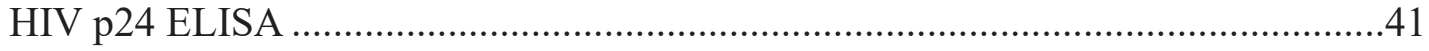

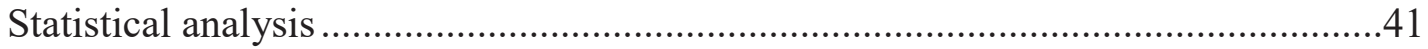

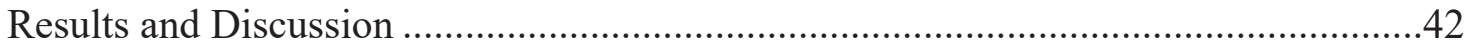

CYP2E1 activity in U937 monocytic and SVGA astrocytic cells ............................42

Effect of acute treatment of DAS analogs on cytotoxicity ...................................43 
Effect on caspase-3 activity .43

Effect of chronic treatment on cytotoxicity with the selected DAS analogs ..............47

Protective effect of the analogs from ethanol-induced toxicity ..................................47

Protective effect of DAS analogs from acetaminophen-induced toxicity...................49

Suppression of viral p24 antigen by DAS analogs ................................................49

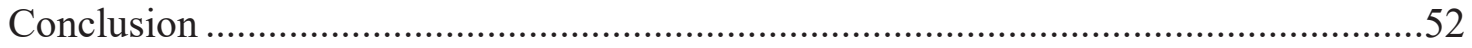

\section{CHAPTER 4. PLASMA EXOSOMES EXACERBATE ALCOHOL- AND} ACETAMINOPHEN-INDUCED TOXICITY VIA CYP2E1 PATHWAY .................53

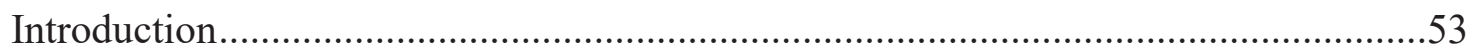

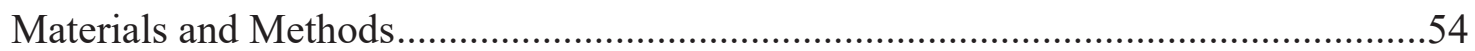

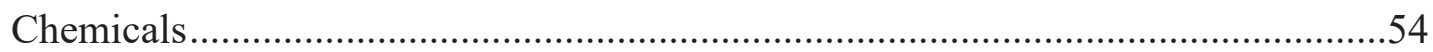

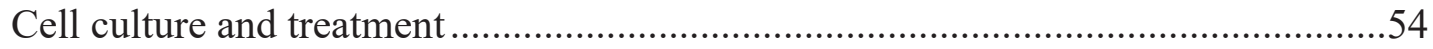

Isolation and validation of exosomes from human and mouse plasma.......................55

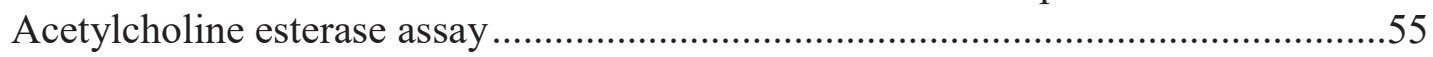

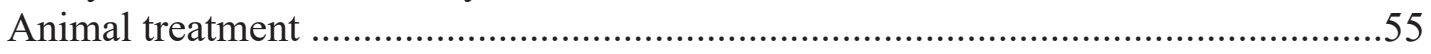

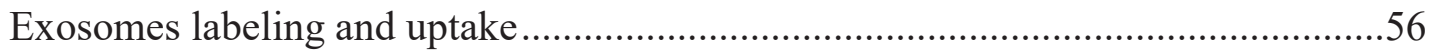

SiRNA transfection and treatment .................................................................56

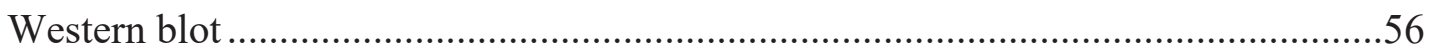

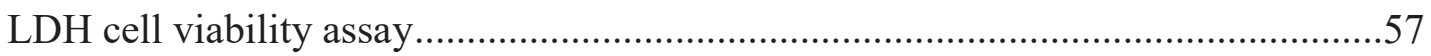

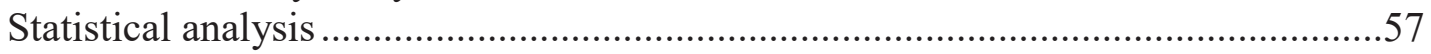

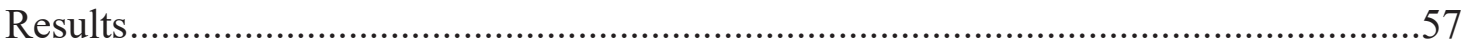

Identification and characterization of plasma exosomes and uptake of exosomes

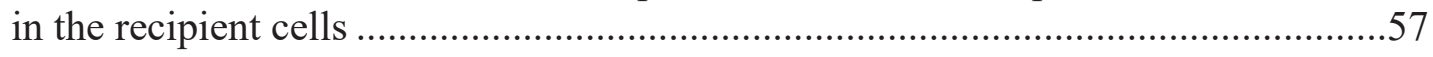

Effect of human plasma exosomes on hepatocytes upon ALC and APAP

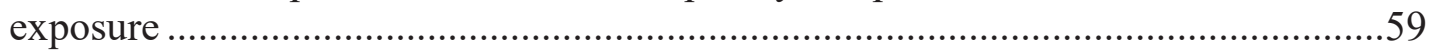

The role of plasma exosomal CYP2E1 in mediating ALC- and APAP-induced

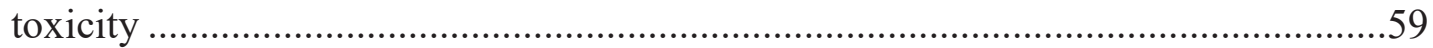

Effect of human plasma exosomes on U1 monocytic cells .......................................61

Effect of alcohol on exosomal CYP2E1 derived from mice plasma ..........................61

Effect of mouse plasma exosomes on U1 cells upon ethanol treatment.....................61

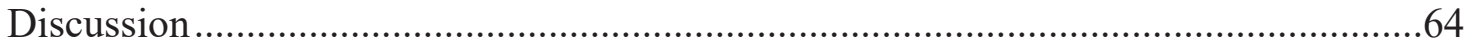

CHAPTER 5. CONCLUSION AND FUTURE DIRECTIONS ...................................68

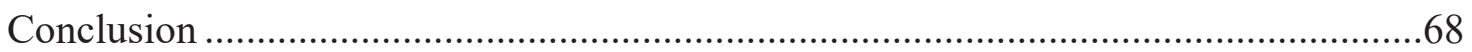

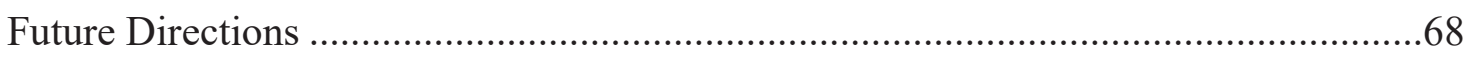

LIST OF REFERENCES ......................................................................................................70

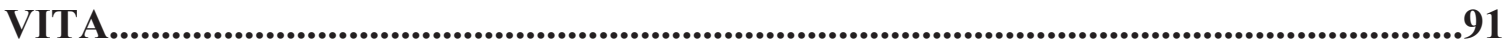




\section{LIST OF TABLES}

Table 1-1. Pathogenic exosomal elements in response to alcohol exposure.................25

Table 2-1. Summary of the CYP2E1 inhibition kinetics of the analogs. .......................33 


\section{LIST OF FIGURES}

Figure 1-1. General pathway of CYP2E1-mediated toxicity and potential benefit and side effects of diallyl sulfide as a CYP2E1 inhibitor.

Figure 1-2. Potential EV/exosomal mechanisms of alcohol-induced toxicity.

Figure 2-1. Structure of the analogs and docking with the CYP2E1 crystal structure....32

Figure 2-2. Inhibition of CYP2E1 activity at varying p-NP substrate and inhibitor concentrations.

Figure 3-1. Effect of acute treatment on cell viability and ROS production in monocytes.

Figure 3-2. Effect of acute treatment on cell viability in monocytes, astrocytes, and hepatocytes.

Figure 3-3. Effect on caspase-3 activity after treatment with DAS and its analogs........46

Figure 3-4. Effect of chronic treatment on cell viability in monocytes and hepatocytes. .48

Figure 3-5. Rescue from ETH-induced toxicity in HepaRG hepatocytes. 50

Figure 3-6. Rescue from APAP-induced toxicity in HepaRG hepatocytes. 51

Figure 3-7. Suppression of viral load by DAS and its analogs in U1 cells. 52

Figure 4-1. Identification of exosomes derived from human plasma. .58

Figure 4-2. Effect of plasma exosomal CYP2E1 in HepaRG cells upon ALC and APAP treatment. .60

Figure 4-3. Rescue of exosomal CYP2E1 mediated toxicity by selective CYP2E1 inhibitor (DE) and CYP2E1 siRNA.

Figure 4-4. Effect of plasma exosomal CYP2E1 in U1 cells upon ethanol treatment. ...63

Figure 4-5. Effect of alcohol on murine plasma exosomal CYP2E1 and AOEs.

Figure 4-6. Schematic representation of the plasma exosomal CYP2E1 mediated toxicity. 


\section{LIST OF ABBREVIATIONS}

$\begin{array}{ll}\text { ADH } & \text { Alcohol Dehydrogenase } \\ \text { AES } & \text { Allyl Ethyl Sulfide } \\ \text { AH } & \text { Alcoholic Hepatitis } \\ \text { ALD } & \text { Alcoholic Liver Disease } \\ \text { ALDH } & \text { Aldehyde Dehydrogenase } \\ \text { AMS } & \text { Allyl Methyl Sulfide } \\ \text { ALC/ETH } & \text { Alcohol/Ethanol } \\ \text { APAP } & \text { Acetaminophen } \\ \text { ART } & \text { Anti-retroviral Therapy } \\ \text { BBB } & \text { Blood-Brain Barrier } \\ \text { CYP } & \text { Cytochrome P450 } \\ \text { DAS } & \text { Diallyl Sulfide } \\ \text { DE } & \text { Diallyl Ether } \\ \text { DMSO } & \text { Dimethyl Sulfoxide } \\ \text { EV } & \text { Extracellular Vesicles } \\ \text { HIV-1 } & \text { Human Immunodeficiency Virus Type-1 } \\ \text { IC } 50 & \text { Half Maximal Inhibitory Concentration } \\ \text { Km } & \text { Michaelis-Menten Constant } \\ \text { MEOS } & \text { Microsomal Ethanol Oxidizing System } \\ \text { NAPQI } & \text { N-acetyl-p-benzoquinone imine } \\ \text { PEA } & \text { 2-(prop-2-en-1-yloxy) ethan-1-amine } \\ \text { PEXA } & \text { 2-prop-2-enoxyacetamide } \\ \text { PLWHA } & \text { People Living with HIV/AIDS } \\ \text { p-NP } & \text { para-Nitro Phenol } \\ \text { ROS } & \text { Reactive Oxygen Species } \\ \text { SiRNA } & \text { Small Interfering RNA } \\ \text { SOD } & \text { Superoxide Dismutase } \\ \text { TP } & \text { Thiophene } \\ \text { WHO } & \text { World Health Organization } \\ 5,1 \text { HA } & \text { 5-hexen-1-amine } \\ & \end{array}$




\section{CHAPTER 1. INTRODUCTION ${ }^{1}$}

\section{CYP2E1-mediated Metabolism and Toxicity}

Human cytochrome P450 (CYP) enzymes are involved in the metabolism of $90 \%$ of the drugs that are currently in use [1]. Among the 57 known functional CYP enzyme families, CYPs 1, 2, and 3 participate in metabolic activation of the majority of foreign substances [2]. Although the liver is the principal site of CYP-mediated metabolism, many extra-hepatic tissues such as the blood, brain, and lungs express CYP enzymes in different magnitudes and participate in drug clearance pathways [3-6]. CYP2E1 is one of the key enzymes which participates in oxidizing low molecular weight substrates, including alcohol (ALC/ETH) and acetaminophen (APAP), along with many environmental pollutants and procarcinogens such as carbon tetrachloride [7-11] $\left(\mathrm{CCl}_{4}\right)$. CYP2E1 is virtually absent in fetal liver, however rapid expression occurs during the postnatal period [12]. In adults, CYP2E1 represents approximately $7 \%$ of total CYP expression [12]. Like all the major CYPs, CYP2E1 is primarily found in hepatocytes and participates in the catalytic conversion of xenobiotics into a more polar form, thereby facilitating their excretion or making them suitable substrates for phase II metabolic enzymes [7]. Expression of CYP2E1 in many extra-hepatic regions such as the brain, gut, kidneys, etc. has been reported in several disease conditions [2]. CYP2E1-mediated production of reactive oxygen species (ROS), coupled with toxic metabolite formation, has been suggested as one of the principal mechanisms of many inflammatory disorders including liver injury [7]. Moreover, CYP2E1 is inducible by its own substrates [13] (e.g. ALC) and also has been found to be upregulated in different pathological conditions [1416] (e.g. human immunodeficiency virus type-1 (HIV-1) infection, diabetes, obesity, cancer, etc.). Moreover, the expression of this enzyme is highly regulated without the presence of any substrate [17]. Therefore, the study of CYP2E1-mediated metabolism is of great pharmacological and toxicological importance.

\section{Epidemiology of ALC- and APAP-related disorders}

ALC is one of the leading etiological factors for several crucial organ disorders and acts as one of the causative factors for more than 200 diseases/injury worldwide [18, 19]. ALC is predominantly metabolized by the liver which is therefore the site of the most damaging consequences of acute and chronic ALC consumption [8, 20]. Alcoholic liver disease (ALD) is a major cause of cirrhosis, acute alcoholic hepatitis (AH), hepatocellular carcinoma, etc. and thus contributes to significant mortality [21, 22]. However, other organs, which metabolizes ALC to certain extent, are also affected adversely upon continuous use [23, 24]. According to the World Health Organization (WHO), it is estimated that harmful use of ALC contributes to $5.3 \%$ ( $\sim 3$ million) of total

\footnotetext{
${ }^{1}$ Portions of this chapter reprinted from final submission with permission from Springer Nature. Rahman M. A., Patters B. J., Kodidela S., Kumar S. Extracellular Vesicles: Intercellular Mediators in AlcoholInduced Pathologies (2019). J Neuroimmune Pharmacol (1557-1904). doi: 10.1007/s11481-019-09848-z.
} 
annual deaths. Moreover, regular ALC consumption promotes disability and relatively early death in comparison to non-drinking populations. Approximately $14 \%$ of total deaths are attributed to ALC-associated disorders in the 20-39 year age group [18]. The causal relationship between ALC drinking and many non-communicable diseases including cancer has been well established [25], and ALC is also associated with several serious infectious diseases such as tuberculosis and HIV/AIDS [26, 27].

APAP is a frequently used over the counter (OTC) analgesic in the USA and worldwide $[28,29]$. Annually, APAP is prescribed to around $6 \%$ of the US adult population with a dose of more than 4 grams/day (recommended total dose is $<4$ grams/day). The main reason behind the popularity of APAP as an analgesic is that, unlike the other nonsteroidal anti-inflammatory drugs (NSAIDs), APAP does not cause significant gastrointestinal toxicity [28]. Unfortunately, severe liver injury, including liver failure culminating in death, can happen if ingested in high doses, either as a result of acute overdose or chronic overuse [29]. APAP can cause significantly more damage in combination with other drugs such as ALC [30]. In fact, the US Food and Drug Administration (US-FDA) has taken numerous steps to raise public awareness with regards to the concomitant use of ALC and APAP/APAP-containing drugs and their potentially fatal impact on the liver [31]. Additionally, overuse of APAP has been associated with disorders of organs other than the liver $[32,33]$. However, the extrahepatic toxicity induced by APAP is less prevalent, and therefore, not extensively reported.

\section{Metabolism of ALC by CYP2E1}

Although ALC possesses nutrient value (about $7 \mathrm{kcal} / \mathrm{gram}$ ), it cannot be stored like other nutrients such as carbohydrates (as glycogen) and fat (as triglycerides) [34]. In fact, it remains mixed with body water until excreted. Moreover, there is negligible hormonal control of ALC metabolism unlike other major nutrients. Hence, the major burden to remove ALC from the body lies on the liver. Unsurprisingly, the enzymes responsible for ALC metabolism are predominantly found in liver cells. There are four enzymes that are involved in the metabolism of ALC: alcohol dehydrogenase (ADH), aldehyde dehydrogenase (ALDH), CYP2E1, and catalase [24]. At lower ALC concentrations, ADH converts ALC to acetaldehyde, which is further oxidized by ALDH to acetate [35]. At elevated concentrations, CYP2E1, the principal CYP enzyme in the microsomal ethanol oxidizing system (MEOS), plays a key role in ALC metabolism which results in generation of reactive free radicals and toxic metabolites [20]. Catalase, on the other hand, oxidizes ALC to acetaldehyde via a hydrogen peroxide $\left(\mathrm{H}_{2} \mathrm{O}_{2}\right)$ dependent pathway in small quantities in the liver [34]. However, catalase along with CYP2E1, is the primary ALC metabolizing-enzyme in the brain [23]. Due to its electrophilic nature, acetaldehyde can bind covalently with DNA, lipids, and proteins which can result in altered cellular homeostasis, conformational changes of proteins, DNA damage and mutation, etc. [35]. 
The MEOS comprises three CYP enzymes- CYP2E1, CYP1A2, and CYP3A4 [36]. However, being the predominant contributor to MEOS-mediated ALC metabolism, CYP2E1 is of significant toxicological importance, especially in chronic and bingedrinking cases. At low concentrations, CYP2E1 may only account for the metabolism of $10 \%$ of total imbibed ALC [34]. The Michaelis-Menten constant (Km) of CYP2E1 for ALC is about $10 \mathrm{mM}$ (10 times higher than that of ADH), which suggests that with increasing ALC concentration, as seen in binge/chronic drinking, the role of CYP2E1 becomes more substantial [34]. Moreover, since CYP2E1 is inducible by ALC, it metabolizes a greater percentage of total ALC at higher concentrations. The mechanism of CYP2E1 induction appears to be predominantly via a post-translational protein stabilization pathway [37, 38]. This is not only true for liver CYP2E1, but also for the extra-hepatic tissues such as kidney, brain, and intestine [39]. Another study has reported that ethanol regulates oxidative stress-mediated CYP2E1 induction via protein kinase C/c-Jun N-terminal kinase/specificity protein 1 (PKC/JNK/SP1) pathway in monocytic and astrocytic cell lines [40]. The increased CYP2E1-mediated ROS and acetaldehyde production results in ALD as well as damage to extra-hepatic tissues.

There are a few CYP2E1 polymorphisms that have been identified, some of which are associated with carcinogenicity by tobacco or other toxins [34]. However, none of these polymorphisms have been linked with the frequency of ALD. The metabolism of ALC in the brain is of particular interest since the exact mechanism of ALC-induced toxicity in the central nervous system (CNS) is not very well characterized. Although catalase has been found to be the principal ethanol-metabolizing enzyme in the brain, growing evidence suggests that CYP2E1 plays a significant role in ALC oxidation to acetaldehyde in the brain $[23,41]$. It is interesting to note that acetaldehyde produced outside the CNS is unable to cross the blood-brain barrier (BBB). Therefore, the levels of acetaldehyde found in the CNS are most likely produced by in situ ethanol metabolism by catalase and CYP2E1 [42-44]. However, chronic ALC exposure has been shown to weaken the integrity of the $\mathrm{BBB}$, which may facilitate external transfer of acetaldehyde into the brain [45]. Recent discovery suggests that intercellular nanovesicles such as exosomes can carry CYP2E1 from the bloodstream through the leaky BBB and contribute to metabolism of ALC in the brain, thereby enhancing ALC-induced neurodegeneration [46-48].

\section{Metabolism of APAP by CYP2E1}

CYP2E1 is responsible for metabolic activation of about 85 xenobiotics which include hepatotoxic and carcinogenic agents [49]. At standard dose (less than $4 \mathrm{gm} /$ day), APAP is primarily converted to pharmacologically inactive glucuronide and sulfate conjugates. A minor fraction (5-10\%) of APAP is oxidized by CYP2E1 (to a lesser degree, by CYP1A2 and CYP3A4) to a reactive metabolite, N-acetyl-p-benzoquinone imine (NAPQI) [50]. NAPQI can bind with the cellular macromolecules such as mitochondrial proteins and ion channels resulting in altered pathophysiology [51]. Normally, NAPQI is detoxified by hepatic glutathione load. However, following a toxic dose, the conjugation pathways of APAP metabolism become saturated, resulting in 
enhanced oxidation by CYP2E1 to toxic NAPQI formation. Due to glutathione depletion at high APAP doses, detoxification of NAPQI is reduced $[50,52,53]$. Increased binding of NAPQI to hepatic proteins is considered to be the primary pathway of APAP-induced hepatotoxicity [9].

APAP overdose surpasses all other etiological factors combined in mediating acute liver failure in the western hemisphere [54]. Usage of CYP2E1 inhibitors such as diallyl sulfide, chlormethiazole, disulfiram, etc. have been suggested as a possible preventative therapy for APAP-induced liver injury [51, 55, 56]. However, their usage is limited by either lower efficacy or potential toxicity [57]. Several xenobiotics such as ALC and isoniazid are known CYP2E1 inducers [58, 59]. Regular co-administration of these drugs with APAP can lead to enhanced NAPQI formation and severe glutathione depletion which may lead to liver injury even at therapeutic APAP concentration [51]. APAP can readily cross the BBB and CYP2E1 in the brain can oxidize APAP to toxic metabolite, NAPQI in situ. This can be particularly harmful for the CNS in the presence of xenobiotics (e.g. ALC) which can induce CYP2E1.

\section{Involvement of CYP2E1 in the Toxicity of Hepatic and Extra-hepatic Cells}

\section{CYP2E1 and hepatotoxicity}

CYP2E1 is involved in metabolism of a wide spectrum of endogenous and exogenous compounds. Just a few of the clinically relevant substrates of CYP2E1 are ALC, APAP, isoniazid, salicylic acid, and $\mathrm{CCl}_{4}$ [60]. Due to remarkably high NADPH oxidase activity, CYP2E1 is capable of producing increased reactive species such as $\mathrm{H}_{2} \mathrm{O}_{2}$, hydroxyl radical, and superoxide anion resulting in oxidative stress $[61,62]$. Oxidative stress is involved in many human diseases including cardiovascular disorders, carcinogenesis, diabetes, Parkinson's disease, heavy metal toxicity, radiation injury, aging, drug-induced toxicity, etc. [61]. Among these, CYP2E1 plays a central role in mediating liver toxicity induced by xenobiotics such as ALC, APAP, and $\mathrm{CCl}_{4}$, predominantly via oxidative stress. The role of oxidative stress in ALC-induced liver injury has been demonstrated convincingly in many studies [39, 60, 63-65]. ALC exposure induces CYP2E1, which in turn oxidizes more ALC, generating further ROS and enhancing lipid peroxidation. Use of CYP2E1 inhibitors and anti-oxidants have been shown to reduce the severity of ALD in in vitro and in vivo studies, which further confirms the role of CYP2E1-mediated oxidative stress in ALC-induced liver injury [6670].

CYP2E1 is also involved in non-alcoholic fatty liver disease (NAFLD), one of the most prevalent chronic liver diseases in the United States [71]. CYP2E1 is not only triggered by foreign xenobiotics but also by endogenous substrates such as polyunsaturated fatty acids which can lead to ROS production and lipid peroxidation [72]. Generally, NAFLD is considered as a comorbidity of obesity and metabolic disorders such as diabetes in which cases CYP2E1 is also found to be significantly 
elevated [73-75]. The most severe form of NAFLD with inflammation is known as nonalcoholic steatohepatitis (NASH). NASH is characterized by hepatomegaly, increased serum aminotransferase levels, and similar histological manifestation as seen in AH [61]. Literatures suggest that CYP2E1 is crucially relevant in the development of NASH due to its ability to induce oxidative stress, inflammatory responses, protein modifications, etc. [76]. In human and rodent NAFLD/NASH models, increased level of CYP2E1 have been found to be well correlated with the severity of liver damage [76].

Metabolism of xenobiotics such as APAP, $\mathrm{CCl}_{4}$, halothane, etc. may result in severe liver injury depending on the extent of abuse. As mentioned earlier, CYP2E1mediated production of NAPQI is the principal mechanism of APAP-induced liver damage. The definitive role of CYP2E1 in potentiating APAP-induced liver injury has been demonstrated in many studies [56]. Similarly, it has been reported that reduction of CYP2E1 expression in the liver prevents the early step of free radical generation by $\mathrm{CCl}_{4}$, a well-known hepatotoxin [77]. CYP2E1-mediated metabolism of $\mathrm{CCl}_{4}$ in liver microsomes results in the formation of reactive metabolites such as trichloromethyl radical $\left(\mathrm{CCl}_{3}{ }^{\circ}\right)$ which can react to membrane lipids and thus cause lipid peroxidation, leading to altered cell structure and function in the liver. 1,2-Dichloroethane (1,2-DCE), otherwise known as ethylene dichloride, is a widely used organic solvent and chemical intermediate that has been found to cause liver toxicity via CYP2E1-dependent metabolic pathway [78]. Many other clinically used drugs such as cisplatin, chlorzoxazone, isoniazid, sodium salicylate, phenobarbital, etc. have been reported to exert hepatotoxic effect via CYP2E1 pathway [79].

\section{CYP2E1 and toxicity in the extra-hepatic tissues}

\section{CYP2E1 and neurotoxicity}

CYP2E1 is involved in several neurodegenerative processes, though the exact mechanistic pathways have not been fully elucidated. In the alcoholic population, it is suggested that CYP2E1 plays a critical role in neuroinflammation, most likely by mediating oxidative damage to mitochondria and cellular proteins [80]. One study showed that ADH- or CYP2E1-mediated metabolism of ALC in human primary neurons produces ROS and nitric oxide (NO) via induction of NADPH/xanthine oxidase and nitric oxide synthase pathway [81]. Increasing ROS and lipid peroxidation correlated with reduced neuronal viability in this study. The same group reported that ALC-induced oxidative stress can cause BBB dysfunction by multiple mechanisms. These mechanisms include myosin light chain (MLC) kinase-mediated phosphorylation of MLC and tight junction proteins, activation of inositol 1,4,5-triphosphate receptor-gated intracellular release of $\mathrm{Ca}^{2+}$, and matrix metalloproteinases activation by tyrosine kinase [82-84]. Increased monocyte migration into the CNS occurs as a result of the dysfunctional BBB, which promotes neuroinflammation [85]. Since oxidative stress acts as a precursor to activation of these pathways, it is highly likely that CYP2E1-mediated oxidative stress in ALC metabolism may be the underlying cause of BBB damage and neuroinflammation. 
Furthermore, polymorphism of CYP2E1 in certain populations have been implicated in Parkinson's disease in several studies [85-87].

\section{CYP2E1 and cancer}

CYP2E1 not only contributes to hepatic toxicity but also can play a role in cancer progression. For example, CYP2E1-mediated mitochondrial oxidative stress and endoplasmic reticulum stress can affect progression and metastasis of advanced breast cancer [88]. Another report suggests that the induction of CYP2E1 and CYP1A1 can play a potential role in pancreatic cancer pathogenesis [36]. Additionally, a CYP2E1 polymorphism has been associated with increasing risk of lung and colorectal cancer [89, 90]. CYP2E1-mediated ALC metabolism results in formation of reactive metabolites. These metabolites can bind to cellular DNA and form carcinogenic exocyclic ethenoDNA-adducts [91]. Increasing evidence suggests significant role of CYP2E1 in chronic ALC-induced carcinogenesis. CYP2E1 metabolizes various other procarcinogens present in environmental pollutants and cigarette smoke to carcinogenic metabolites [91].

Moreover, several polymorphisms of CYP2E1 have been linked with chemically-induced cancers in many studies [92].

\section{Involvement of Oxidative Stress and CYP2E1 in HIV-1 Pathogenesis}

It is well recognized that increased oxidative stress induces a plethora of pathological conditions [93-95], and HIV-1 pathogenesis is no exception[96]. Even without an external trigger, the HIV viral proteins such as the transactivator of transcription (Tat) protein, the envelope glycoprotein gp120, etc. have been shown to induce oxidative stress in people living with HIV-1/AIDS (PLWHA) [57, 97, 98]. The persistent oxidative imbalance is due to the weakening of the host antioxidant defense machinery, as manifested by reduced glutathione, catalase, superoxide dismutase (SOD), and S-methyl transferase levels [57]. As a result of this reduced antioxidant capacity along with increased inflammatory/immune responses, the human body suffers from enhanced HIV pathogenesis as well as opportunistic infections such as tuberculosis (TB) $[99,100]$. It is important to note that there are approximately 14 million people living with HIV-TB coinfection worldwide [101]. ALC drinking is another significant co-factor that contributes to enhanced HIV-1 replication and toxicity of various vital organs such as the liver and brain [102-104]. It is estimated that roughly $50 \%$ of the PLWHA are regular consumer of ALC [57]. This not only affects adherence to anti-retroviral therapy (ART), but also increases ART-induced hepatotoxicity, partially due to inefficient metabolism of these drugs [105]. Furthermore, concurrent intake of ALC and analgesic drugs such as APAP can lead severe glutathione depletion due to enhanced oxidative stress which can exacerbate HIV-1-inducedimmune dysregulation [106].

Due to the advent of highly active ART medication, HIV/AIDS is no longer a life-threatening condition. However, in chronic HIV-1 infection, delayed access and lack of adherence to ART medication coupled with inability of these drugs to cross the BBB can lead to HIV-associated neurocognitive disorders (HAND) in PLWHA [107, 108]. 
Especially, ALC abuse and HIV-1 infection in combination poses a major threat to PLWH in this regard [109, 110]. The most severe form of HAND is known as HIV-1 associated dementia (HAD) $[108,111]$. Although, the exact mechanism of HAD is not clearly defined, oxidative stress has been identified as one of the key factors in this condition $[96,112]$. One study reported that, in the brains and cerebrospinal fluid (CSF) of people with HAD, increased oxidative stress components were present [113].

Additionally, the use of antioxidants in ex vivo experiments resulted in reduced oxidative stress associated toxicity. Another study reported that ALC abuse and HIV-1 infection promoted oxidative stress and mitochondrial depolarization in primary human astrocytes via a CYP2E1-dependent pathway [114], which contributes to increased cytotoxicity. In many studies, ALC-induced overexpression of CYP2E1 leading to further ALC metabolism and oxidative stress has been reported in hepatocytes, monocytes/ macrophages, and astrocytes [115-117]. Most importantly, similar results have been observed in HIV-1 infection both in in vivo and in vitro systems [118, 119]. Taken together, ALC-induced CYP2E1-mediated oxidative stress can lead to poor ART metabolism due to hepatoxicity, which in turn can facilitate additional HIV-1 replication resulting in neurocognitive disorders such as HAND and HAD.

From the reports discussed above, it is clear that CYP2E1 mediated metabolism and resulting oxidative stress play a crucial role in xenobiotics- and disease-induced toxicity. One of the fundamental corrective measures that has been proposed is to boost the cellular antioxidant defense system, which can be done by using established antioxidants or by inhibiting CYP2E1-dependent metabolic pathways. Unfortunately, there are no safe and selective CYP2E1 inhibitors available to use in these pathophysiological conditions. Also, the exact mechanistic pathway for CYP2E1mediated toxicity, especially in extra-hepatic regions, needs to be further investigated in order to develop appropriate therapeutic regimens.

\section{Potential of Diallyl Sulfide as a CYP2E1 Inhibitor}

Diallyl sulfide (DAS, $\mathrm{C}_{6} \mathrm{H}_{10} \mathrm{~S}$ ) is a lipophilic thioether most commonly found in garlic (Allium sativum). Consumption of garlic for its medicinal values has been practiced for more than 3000 years. DAS is one of the principal organosulfur compounds present in garlic imparting its anti-cancer, antioxidant, analgesic, and anti-inflammatory properties [120]. When garlic is sliced or ground, the chief organosulfur compound, allin is converted to allicin by the vacuolar enzyme alliinase. Allicin undergoes spontaneous degradation and produces DAS (molecular weight: $114.2 \mathrm{~g} / \mathrm{mol}$ ) along with many other oliferous compounds including diallyl disulfide (DADS) and diallyl trisulfide (DATS) [121]. DADS and DATS have been reported to have beneficial effects in several studies $[122,123]$, but DAS is the most extensively studied component of garlic due to its wide range of therapeutic applications. Among the manifold activities of DAS, inhibition of CYP2E1 enzyme has the most therapeutic potential and clinical relevance. 


\section{Protective Effects of Diallyl Sulfide (DAS)}

\section{Anti-cancer effect}

Oxidative stress can trigger profound alterations in cellular pathophysiology and thus plays a critical role in numerous malignancies. Supply of an external antioxidant agent therefore can be of great significance in oxidative stress-induced pathologies, especially in chronic diseases. Several studies have shown that DAS treatment resulted in significant upregulation of several antioxidant enzymes (AOEs) such as SOD, catalase, glutathione reductase, etc. [124, 125]. Reduced glutathione (GSH) is an important free radical/ROS scavenger, and its ratio with oxidized glutathione (GSSG), known as the GSH:GSSG ratio, is considered as an indicator for oxidative stress [126]. In rats, DAS treatment has shown enhanced GSH:GSSG ratio, indicating improved redox homeostasis [125]. DAS-mediated upregulation of AOEs has been shown to be associated with the transcription factor nuclear factor (erythroid-derived 2)-like 2 (Nrf2) pathway.

\section{Anti-inflammatory effect}

Anti-inflammatory properties of DAS have been reported in multiple studies. In rat aortic smooth muscle cells, DAS reduced tumor necrosis factor (TNF)- $\alpha$ and histamine-induced inflammatory responses [127]. DAS has also been found to modulate the GSH redox cycle and inhibit the activation of nuclear factor kappa B (NF-kB) in human T cells [121]. Moreover, in another study, it was observed that DAS reduced bleomycin-induced activation of inducible nitric oxide synthase and the levels of inflammatory cytokines, NF- $\mathrm{KB}$, and interleukin- $1 \beta$ in lung tissue [128]. Beneficial effects by DAS were also observed in osteoarthritis patients, in whom DAS blocked cyclooxygenase-2 upregulation by attenuating NF-KB activation [129].

\section{Effect on drug metabolizing enzymes}

Several organosulfur compounds in garlic have been found to induce phase I and II drug metabolizing enzymes [120]. DAS treatment has been shown to selectively induce CYP3A1/2 and CYP2B1/2 [130]. Moreover, increased activity of the phase II enzymes UDP-glucuronosyltransferase, microsomal epoxide hydrolase, and glutathione-Stransferase were reported in DAS-treated rats compared to control animals [131]. However, the most significant interaction of DAS among the drug metabolizing enzymes is with CYP2E1.

\section{DAS as CYP2E1 Inhibitor}

DAS has been studied for its inhibitory action on CYP2E1-mediated metabolism

of many toxic xenobiotics. Several studies strongly suggest its role as a CYP2E1 inhibitor 
and as a potential therapeutic or adjunctive therapy in CYP2E1-mediated pathologies. The protective role of DAS as a CYP2E1 inhibitor in ALC-, and APAP-induced toxicity as well as in HIV-1 comorbidity is discussed below.

\section{Protection against ALC-induced toxicity}

CYP2E1 dependent metabolism of ALC and resulting oxidative stress is one of the principal etiological factors for hepatic and non-hepatic toxicity in chronic and binge drinking populations. DAS, by inhibiting CYP2E1-mediated metabolism of ALC, has shown protective effects in both in vitro and in vivo studies. For example, DAS treatment partially protected primary human hepatocytes from ALC-induced cellular damage by inhibiting CYP2E1 [132]. In another study, feeding rats with DAS reversed ALC-induced changes in CYP2E1-dependent fatty acid metabolism and lipid peroxidation, which resulted in improved liver function [133, 134]. Similarly, in chronic ethanol treated human hepatoma cell line, DAS rescued the cells from CYP2E1-mediated oxidative stress, toxicity, and aldehyde-protein adduct formation [135]. In a study conducted by the Kumar group, it was observed that DAS pretreatment abolished ALC-induced ROS, DNA damage, and cellular toxicity in monocytic and astrocytic cells [115]. A similar effect was observed in ex vivo rat brains, where DAS pretreatment significantly reduced acetaldehyde and acetate formation [136]. A recent study reported beneficial effects of DAS in ALC-stimulated alcoholic cardiomyopathy. Taken together, it is likely that DAS can be an effective tool in alleviating ALC-induced oxidative stress and organ damage.

\section{Protection against APAP- and other drug-induced toxicity}

Overdose of the popular analgesic, acetaminophen, is one of the leading causes of acute liver injury in the world. A recent report showed that even a standard APAP dose can be associated with liver failure [137]. It is well established that CYP2E1 plays the key role in APAP-induced toxicity. Administration of DAS prior to or shortly before APAP treatment significantly reduced liver injury in rat model [138]. In an in vitro study using CYP2E1 transfected HepG2 cells, DAS showed cytoprotective effects by inhibiting CYP2E1 mediated oxidation of APAP [139]. Another report demonstrated that DAS lowered the synergistic toxicity caused by arachidonic acid and salicylate, which is an anti-inflammatory and analgesic agent and a substrate of CYP2E1 [140]. These findings suggest the ameliorating role of DAS as a CYP2E1 inhibitor in APAP- and other druginduced toxicity.

\section{Protection from HIV-1 comorbidity}

Drug abuse is very common phenomenon in PLWHA. Nearly half of the PLWHA with HIV are regular alcohol drinkers. The HIV protein gp120 has been shown to be associated with increased CYP2E1 expression which can worsen ALC-induced hepatoxicity in this group. One study reported that CYP2E1 is involved in gp120 and 
methamphetamine (MA)-induced oxidative stress in astrocytes. DAS treatment significantly reduced gp120/MA-induced ROS production and apoptotic cell death in those cells [141].

\section{Metabolism of DAS by CYP2E1 and Potential Toxicity}

DAS is not only an inhibitor of CYP2E1, but metabolic conversion of DAS also happens via the CYP2E1 pathway $[142,143]$. The metabolites of DAS are diallyl sulfoxide (DASO), diallyl sulfone ( $\left.\mathrm{DASO}_{2}\right)$, and allyl mercaptan. The major two metabolites DASO and $\mathrm{DASO}_{2}$ are formed by S-oxidation via CYP2E1, while allyl mercaptan is a spontaneous breakdown product of DAS. These are further converted into respective epoxides which are toxic to hepatic and extra-hepatic cells. Additionally, DAS itself can undergo direct epoxidation which is also toxic. Further, the oxidation of DAS causes autocatalytic destruction of the CYP2E1 enzyme. These phenomenon acts as a roadblock for DAS to be considered for actual therapeutic use. Therefore, there is a critical need for designing and developing novel DAS analogs which will retain the CYP2E1 inhibitory characteristics of DAS but with reduced ability to act as a CYP2E1 substrate.

Despite showing protective effects in ALC-, APAP-, and other xenobiotic/disease-induced toxicities by blocking CYP2E1-mediated metabolism, due to its rapid metabolism by CYP2E1 to toxic metabolites, usage of DAS for chronic study design or as a potential therapeutic modality is similarly limited. Figure 1-1 illustrates this phenomenon.

\section{Exosomes as Intercellular Mediators in Alcohol-induced Pathologies}

According to the World Health Organization (WHO), alcohol-related complications pose a major public health challenge to the world population, contributing to approximately $5 \%$ of the global burden of disease and $6 \%$ of total deaths annually [144]. Alcoholic liver injury, which includes hepatitis and cirrhosis, majorly contributes to this problem. In addition to liver damage, excessive alcohol consumption can cause severe damage to the central and peripheral nervous systems, the gastrointestinal tract, the heart and vascular systems, and the endocrine and immune systems [145]. The WHO reports that drinking alcohol is associated with more than 60 non-communicable diseases, including cancer. Moreover, recent evidence points to a causative association between alcohol intake and infectious diseases such as HIV-1, tuberculosis, and pneumonia [144]. For many years, investigators have undertaken research studies to understand these alcohol-induced hepatic and extra-hepatic complications [146, 147]. However, the molecular and cellular mechanisms of alcohol-induced toxic effects follow a multiplicity of pathways. Despite tremendous advancements in the study of alcohol metabolism and its effects, the complete mechanism(s) by which alcohol causes tissue injury, especially extra-hepatic complications, are still poorly understood. 


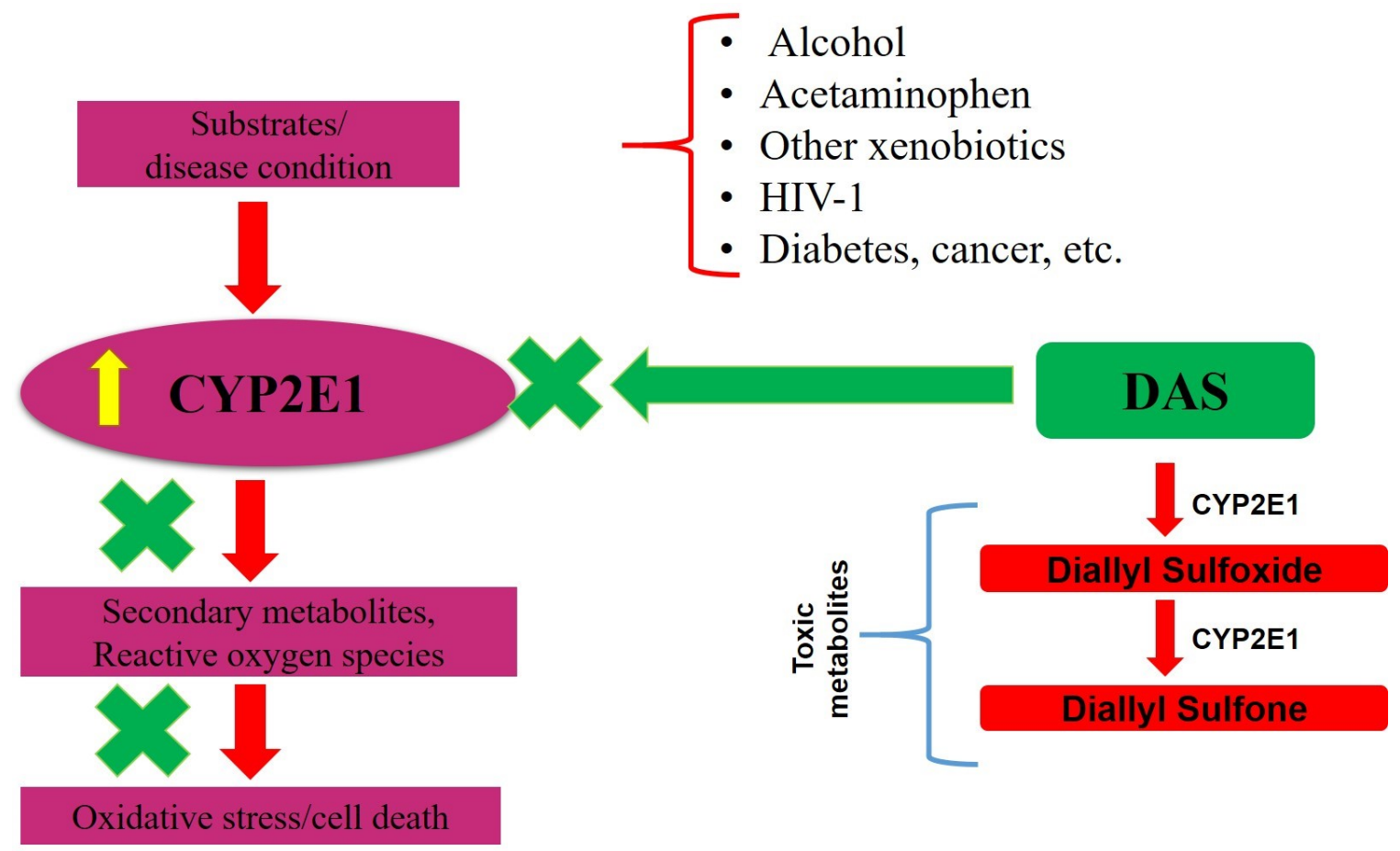

Figure 1-1. General pathway of CYP2E1-mediated toxicity and potential benefit and side effects of diallyl sulfide as a CYP2E1 inhibitor.

Modified with permission from Bentham Science Publishers. Rao P.S.*, Midde N.M.*, Miller D.M., Chauhan S., Kumar A., Kumar S. Diallyl Sulfide: Potential Use in Novel Therapeutic Interventions in Alcohol, Drugs, and Disease Mediated Cellular Toxicity by Targeting Cytochrome P450 2E1 (2015). Curr Drug Metab, 16(6), 486-503. (* Co-first author). doi :10.2174/1389200216666150812123554. 
One potential phenomenon that is likely to play a role in alcohol-associated pathologies, but has yet to be extensively studied, is the formation and release of extracellular vesicles (EVs). EVs were first identified more than 50 years ago by two different groups $[148,149]$. At that time, they were referred as "platelet dust" or "procoagulant platelet-derived particles". These vesicles, small membrane-bound microparticles containing proteins, RNA, and other biomolecules from their donor cell, have only attracted mainstream scientific attention relatively recently. There are mainly three distinct type of EVs- exosomes, microvesicles, and apoptotic bodies which are secreted from eukaryotic cells [150]. These are categorized based on the size, mechanism of biogenesis, and their biological contents. Exosomes are produced from multivesicular bodies via endosomal pathway which includes internal budding and exocytosis [151]. Multivesicular bodies contain intraluminal vesicles which contains cellular proteins, lipids, RNAs, etc. [152]. Inside cell cytoplasm, the intraluminal vesicles are formed by components of the endosomal-sorting-complex-required-for-transport (ESCRT) machinery, lipids, and tetraspanins (e.g. CD63, CD81, etc.). When the multivesicular bodies dock and fuse with the plasma membrane, the intraluminal vesicles are released as exosomes [152]. The size of exosomes typically varies from 30-150 $\mathrm{nm}$. One the other hand, microvesicles are generated as a result of outward budding off the plasma membrane and the size ranges typically from 100-1000 nm. The biological contents of microvesicles are quite similar to that of exosomes, mechanism of biogenesis being the major distinction between these two EV types. Apoptotic bodies are largest among all the EVs (size ranges from 1-5 $\mu \mathrm{M}$ ) and are produced as a result of cell fragmentation/ blebbing during programmed cell death. Apoptotic bodies are sometimes referred as apoptosomes [153]. In general, EVs have a short half-life (from a few minutes to $\sim 6 \mathrm{~h}$ after their release into the circulation), probably because of their subsequent uptake into the recipient cells $[152,154]$. In the current review, EV has been used as a generic term which encompasses exosomes, microvesicles, and apoptosomes. However, it is important to note that the role of exosomes has been discussed in more detail in the latter part of this manuscript due to their greater clinical and biological implications.

Among all the EVs, exosomes in particular, has become a new buzzword in the last decade, due to their relatively tightly regulated biogenesis and packaging processes. Interestingly, when they first emerged on the scene in the late 1980s, exosomes were thought to be the "garbage cans of the cell", i.e. their function was assumed to be removal of unnecessary proteins and other cellular debris [155]. Dr. Rose Johnstone from McGill University, who was among the first to identify exosomes, believed that they could act as key regulators in cellular processes, though exosomes research has gained significant momentum only in the last decade $[156,157]$. Due to advances in exosome isolation techniques and proteomic analysis, emerging evidences have revealed that these oncecalled "dumpsters of cells" are crucial mediators of both cell-to-cell communication and disease pathogenesis [155].

Recent findings show that EVs/exosomes can shuttle a plethora of key biological agents such as microRNA (miRNA), mRNA, lipids, proteins, and other molecules through biological fluids to both nearby recipient cells and those at distant sites [158, 159]. Due to the tightly regulated mechanisms of cargo sorting and process of vesicular 
biogenesis, the cargo of EVs/exosomes generally varies between healthy and diseased populations, suggesting a role for EVs/exosomes in mediating cellular communication and disease progression [160]. EVs have been shown to play significant roles in the innate immune response, tumor progression, angiogenesis, and other processes, and recent data intriguingly suggest that alcohol can modulate the EV/exosomal pathway in both hepatic and extra-hepatic tissue systems, potentially exacerbating these effects [161163]. This phenomenon can potentially help us to decipher the missing 'pieces of the puzzle' of the broad spectrum of alcohol-induced toxic outcomes in various tissues. In this review, we have attempted to summarize the latest discoveries relating to the effect of alcohol on secretion of EVs, with a major focus on exosomes, specific packaging of biomolecules and their transport via EVs/exosomes, and the potential impact of alcoholmodified EVs/exosomes on the recipient cells.

\section{Alcohol-induced EVs/exosomes from Hepatic Cells in Cell-cell Communication}

The liver is the principal organ responsible for the metabolism of most xenobiotic compounds, including ethanol, often estimated to metabolize up to $90 \%$ of imbibed ethanol [8]. It is equipped with high concentrations of the three major ethanolmetabolizing enzymes: alcohol dehydrogenases (ADHs), cytochrome P450 2E1 (CYP2E1), and catalase. Each of these enzymes metabolizes ethanol to acetaldehyde, which causes DNA and protein adducts that greatly contribute to the risk of liver cancer [164]. Additionally, the CYP2E1 enzyme, in the process of metabolizing ethanol to acetaldehyde, produces superoxide anion and hydrogen peroxide. These reactive oxygen species (ROS) are agents that have long been known to cause oxidative liver damage [8, 165]. These common metabolic pathways of ethanol are generally well understood. Even so, alcohol still acts as a major risk factor in many disease conditions aside from the widely heralded phenomenon of alcohol-induced liver injury. Since the ethanol molecule displays tremendous biological reactivity, the consequences of alcohol abuse can be farreaching and complex. Therefore, alcohol-induced extra-hepatic complications remain a major area of interest today. Recent studies strongly point to the potential involvement of a defined group of biological nanovesicles, namely exosomes, as a key player in modulating the deleterious effects of alcohol in different tissue systems. Cho et al. [166] reported that exosomes obtained from mice with acetaminophen-induced liver injury can cause hepatotoxicity in recipient naïve primary hepatocytes as well as in mice. Exosome treatment caused elevation of plasma reactive oxygen species in mice and increased the expression of proteins associated with apoptotic signaling pathway such as phosphoJNK/JNK, Bax, and cleaved caspase-3 in the recipient hepatocytes. Alcohol-induced liver toxicity may follow a similar exosome-mediated communication pathway. In the following section, we attempt to summarize the findings from the recent publications regarding changes to liver-derived EVs/exosomes upon alcohol exposure and their potential role in cellular communication, especially between hepatocytes and immune cells such as monocytes. 


\section{Hepatic EVs/exosomes in alcoholic hepatitis}

Alcoholic hepatitis ( $\mathrm{AH})$ is one of the most devastating conditions associated with heavy alcohol intake, manifesting as acute inflammation of the liver [167]. AH pathogenesis follows a multifactorial pathway that involves intricate interplay between metabolism of alcohol, liver damage, and inflammation. The identification and role of

different inflammatory mediators such as tumor necrosis factor $\alpha(\mathrm{TNF} \alpha)$ and interleukin$1 \beta$ (IL-1 $\beta$ ) in AH and gut microbiome-derived lipopolysaccharides (LPS) have been studied extensively [168]. Damage to hepatocytes has been recently shown to be a prerequisite of alcohol-induced liver inflammation [169]. However, many facets of this pathogenesis remain unclear. For example, the exact mechanism of cross-talk between hepatocytes and immune cells (such as monocytes, macrophages, T-cells, etc.) was unknown until recently. Accumulating evidence suggests the potential role of exosomal miRNAs in drug-induced liver, kidney, and muscle injury [170-173].

MiRNAs play a fundamental role in regulating AH pathogenesis. One recent study reported that miR-122, miR-192, and miR-30a can act as useful diagnostic markers for AH [174]. MiR-122 is abundantly expressed in hepatocytes, but to a much lower degree in immune cells, and its function remains unclear. Momen-Heravi et al. [175] observed that, after chronic and/or binge alcohol exposure, greater numbers of exosomes that were rich in miR-122 were produced in human sera. They also demonstrated that alcohol exposure increased miR-122-enriched exosome production in hepatocytes in a dose-dependent manner. Most importantly, these exosomes horizontally transported miR122 to monocytes, which rendered them more sensitive to LPS stimulation, inhibited heme oxygenase-1, and enhanced secretion of proinflammatory cytokines. Pre-treatment with exosomes loaded with a miR-122 inhibitor prevented this proinflammatory phenotype. In brief, this group demonstrated that exosomal transfer of miR-122 and consequent immune modulation could potentially be an alternative pathway of immune sensitization to LPS in AH pathogenesis. In addition, this study also demonstrates the potential for use of exosomes as an effective vehicle for delivering gene and RNA interference therapy in immune cells to reverse exosome-mediated deleterious effects from alcohol-exposed hepatocytes.

\section{Hepatic EVs/exosomes in alcoholic liver disease}

Alcoholic liver disease (ALD) is an umbrella term for a broad spectrum of disorders which includes AH, with or without cirrhosis, steatosis, hepatoccellular carcinoma, etc. Due to the varying spectrum of ALD, the pathophysiology is incompletely understood and hence, it is one of the leading causes of chronic liver disease [176]. A recent study by Verma et al. [177] attempted to demonstrate the mechanism by which macrophages are activated following alcohol exposure to hepatocytes in alcoholic liver disease (ALD). The authors showed that ethanol treatment significantly increased caspase- 3 activation which triggered increased EV production in hepatocytes. However, blocking of caspase-3 activation abolished the alcohol-induced EV production, which indicates that alcohol triggers enhanced vesicle production via a 
caspase-dependent pathway. Moreover, they also showed that cluster of differentiation 40 ligand (CD40L), a member of the TNF superfamily, was highly packaged in exosomes upon alcohol exposure (in vitro, in vivo, and ex vivo) and was associated with increased inflammatory cytokine production as well as hepatic macrophage infiltration. Blocking of CD40L reversed those phenomena, indicating that the crosstalk between hepatic and immune cells upon ethanol exposure may occur through a CD40L-mediated pathway.

A similar study conducted by Saha et al. [178] showed that EVs isolated from ALD mice had protein cargos distinct from the non-ALD mice, and that EV-induced activation of macrophages was mediated by heat shock protein 90 (Hsp90). Hsp90 is a molecular chaperone protein that has been previously shown to be involved in macrophage activation by the anti-tumor agent Taxol and by bacterial LPS [179]. This study demonstrated that upon intravenous administration of ALD-derived EVs into alcohol-naïve mice, those EVs were uptaken by recipient hepatocytes and macrophages. The EV-treated hepatocytes showed increased expression of monocyte chemoattractant protein 1 (MCP1) compared to hepatocytes treated with control EVs. MCP1 is an important chemokine that regulates recruitment of monocytes/macrophages in response to inflammation [180]. Moreover, the authors observed significant induction of Hsp90 in whole liver, and in purified hepatocytes isolated from ALD mice compared to pair-fed mice. The authors hypothesized that this elevated amount of Hsp90 was highly likely to be secreted via hepatic EVs into systemic circulation. Proteomic analysis showed that ALD EVs did indeed have higher expression of Hsp90. Furthermore, treatment of macrophages with recombinant Hsp90 resulted in a dose-dependent increase in the expression of the pro-inflammatory cytokines TNF- $\alpha$ and IL-1 $\beta$, whereas the level of anti-inflammatory markers such as CD163 and CD206 were reduced. Inhibition of Hsp90 by a competitive inhibitor reversed this phenomenon, confirming the role of Hsp90 in macrophage activation in ALD via an EV-mediated pathway.

\section{Hepatic EVs/exosomes in fibrogenesis}

In recent years, liver-derived exosomes have also been implicated in wound healing mechanisms. For example, Nojima et al. [181] observed that in the case of ischemia/reperfusion injury or partial hepatectomy, exosomes derived from primary murine hepatocytes induced hepatocytic proliferation by fusing with recipient liver cells and delivering neutral ceramidase and sphingosine kinase 2, resulting in increased production of sphingosine-1-phosphate (S1P). S1P is a critical regulator in many pathophysiological processes, including cell proliferation [182]. The number of exosomes with proliferative effects increased after ischemia/reperfusion injury. In this scenario, hepatic exosomes appeared to play a beneficial role in response to tissue injury. Though, that is not true for all disease conditions. For example, exosomes may play an important role in tissue fibrogenesis, which is a natural physiological response to stress-induced tissue injury. However, several tissue repair processes are activated during stress conditions, and chronic insults such as prolonged ethanol exposure lead to dysregulation of the wound healing process, resulting in tissue fibrosis, scar formation, and ultimately organ failure. Alcohol metabolism triggers the release of a major fibrogenic cytokine, 
transforming growth factor-beta-1 (TGF- $\beta 1$ ), and hepatic stellate cell (HSC) activation, which are key events in fibrosis progression [183]. These changes coincide with the dysregulation of global miRNA expression in liver cells, which play crucial roles in HSC functionality. Although the role of cellular miRNA-mediated HSC activation in alcoholic fibrosis has been well studied, the potential contribution of exosomal miRNAs in this phenomenon has not been delineated yet. In one recent study, Brandon-Warner et al. [184] found that alteration of a single miRNA, miR-19b, can result in a change in the expression and localization of multiple other miRNAs at the cellular and exosomal levels in an alcohol-induced hepatic fibrogenesis model. MiR-19b is part of the miR-(17-92) cluster family, and has been shown to be downregulated in activated HSCs. In addition, alcohol increased the expression of pro-fibrotic genes and decreased miR-19b level in HSCs. Interestingly, the level of miR-19b was significantly induced in plasma- and activated HSC-derived exosomes. However, the clinical relevance of the release of exosomal miR-19b in the fibrogenic pathway needs additional investigation.

\section{Alcohol and EVs/exosomes Derived from Extra-hepatic Tissues in Cell-cell Communication}

Alcohol not only affects the liver, but also myriad other tissues. As mentioned previously, numerous studies have focused on the effects of alcohol on hepatocytes and liver-derived exosomes. However, very few studies exist concerning EVs/exosomes derived from ethanol-treated non-hepatic cells and tissues. In the following section, we summarize the findings of those studies that have been conducted on the effect of alcohol on the secretion as well as content of EVs/exosomes derived drom extra-hepatice cells. This information will contribute to our understanding of the role of alcohol in altering EV/exosome-mediated intercellular communication, which in turn may lead to novel therapies for alcohol-induced extra-hepatic toxicities.

\section{Alcohol and EVs/exosomes in the immune system}

Alcohol can increase release of EVs from immune cells such as monocytes [185]. Monocytes are differentiated into macrophages in response to various stimuls/signals. These macorphages can polarize to either M1 (proinflammatory) or M2 (inflammatory) phenotypes depending upon the type of stimulus/status of the disease. Saha et al. [185] found that exosomal miRNA-27a plays a role in the process of macrophage differentiation and/or polarization. Authors have demostrated that exosomes derived from ethanol-treated monocytes stimulate naïve monocytes to differentiate into M2 macrophages, mediated through miR-27a (an M2-polarizing miRNA). The authors validated these findings in vivo: circulating EVs in the plasma of alcoholic hepatitis patients were found to have high expression of miR-27a [185]. Studying the transport of miR-27a by exosomes derived from monocytes may give insights into the role of exosomes in mediating the modulatory effects of ethanol on the mechanisms of inflammation. 
Similarly, alcohol can also stimulate certain cells of the immune system to relase cytokines [186] and these can be packaged in exosomes [187]. A recent publication from our own laboratory investigating the plasma and exosomal cytokine levels in substance abusers and HIV-infected subjects found that chronic alcohol comsumption could alter the exosomal packaging of cytokines in vivo [187]. In particular, IL-10 is highly packaged in the plasma exosomes of alcohol drinkers, though whether or not the higher exosomal levels of this anti-inflammatory cytokine reflect a physiologically protective or harmful phenomenon in chronic drinkers is a matter still under investigation.

\section{Alcohol and EVs/exosomes in epithelia and endothelia}

Ethanol also increases EV production in a dose-dependent manner from endothelial cells derived from human umbilical vein (HUVECs) and dermal microvasculature (HDMECs), according to a recent publication by Lamichhane et al. [188]. Further, exposure of naïve endothelial cells to exosomes derived from ethanoltreated endothelial cells increases vascularization by down-regulating anti-angiogenic miRNA-106b and up-regulating the pro-angiogenic long non-coding RNAs MALAT1 and HOTAIR [188].

Similarly, Atienzar-Aroca et al. [189] reported that retinal pigment epithelium (RPE) cells also dose-dependently produced higher quantities of exosomes upon exposure to ethanol. Further, these exosomes contained higher levels of vascular endothelial growth factor receptor (VEGFR-1 and VEGFR-2) mRNA than control exosomes. Interestingly, when exosomes derived from ethanol-treated RPEs and untreated control cells were exposed to HUVECs, the progression of tube formation was delayed by control exosomes and enhanced by ethanol-modified exosomes. Moreover, the intracellular levels of VEGFR-1 and VEGFR-2 were also increased in recipient HUVECs upon treatment with exosomes derived from the ethanol-exposed RPEs [189]. These findings indicate that EVs can be used as therapeutic targets for alcohol-induced angiogenesis in pathological conditions.

Alcohol is a known risk factor of oral squamous cell carcinoma (OSCC). Recently Momen-Heravi et al. [190] found altered miRNA expression in plasma EVs of patients with OSCC compared to healthy patients, as well as higher total EV content. Further, stimulation of an OSCC cell line with different doses of alcohol stimulated release of EVs containing known oncogenic miRNAs, particularly miR-21. Treatment of monocytes with EVs derived from OSCC cell lines transfered this miR-21, leading to activation of the NF-kB pathway and stimulating production of MCP-1. These effects were more pronounced with the administration of EVS from OSCC cells treated with alcohol, further supporting the hypothesis that regular alcohol consumption may exarcerbate oral cancer and provoke excessive inflammation. 


\section{Alcohol and EVs/exosomes in the heart}

Cardiac myocytes are not particulalry secretory cells. However, they can release exosomes upon exposure to certain stimulus or treatments [191]. Malik et al. [191] observed that exosomes derived from ethanol-treated cardiac myocytes had different protein content compared with exosomes derived from hypoxia/reoxygenationexperienced cells. Specifically, several proteins in ethanol-derived exosomes were found to be mitochondrial in origin. Since mitochondrial dysfunction is known to play a major role in oxidative stress, and heavy ethanol consumption contributes to cardiomyopathy through oxidative stress [192], exosomes derived from alcohol-exposed cells are likely to transport mitochondrial elements that could contribute to oxidative stress-induced intercellular signaling. Also, as with other tissues mentioned previously, ethanol increased exosome production from the cardiac myocytes via oxidative stress. Ethanol exposure, variations in $\mathrm{pH}$, low temperature $\left(4^{\circ} \mathrm{C}\right)$, and hypoxia/reoxygenation conditions did not alter the stability and membrane permeability of the myocytic exosomes [191] suggesting that they retain their protein cargo under diverse physiological/pathological conditions.

\section{Alcohol and EVs/exosomes in the pancreas}

Pancreatic stellate cells (PSC), upon exposure to ethanol, become activated and release connective tissue growth factor (CCN2), which regulates collagen deposition and fibrogenesis, in a dose-dependent manner. In a murine model of alcoholic chronic pancreatitis, co-treatment of ethanol with the compound cerulein (an inducer of pancreatitis) induced both CCN2 and miR-21 expression. Further, it was shown that, in the activated PSCs, CCN2 not only increases collagen deposition but also induces miR21, which in turn increases CCN2 expression by a positive feedback mechanism [193]. The authors, Charrier et al. [194], have also demonstrated that exosomes derived from PSCs package CCN2 mRNA and miR-21 proportionally to their relative cellular expression, and that these exosomes are successfully delivered to recipient PSCs. The exchange of RNA elements from one cell to another upon stimulation with ethanol indicates that chronic drinking perhaps exacerbates existing pathological conditions such as pancreatic fibrosis.

\section{Plasma exosomes and cytochrome P450 enzymes}

The presence and biological significance of drug metabolizing cytochrome P450 (CYP) enzymes in EVs especially in exosomes have not yet been investigated in detail. In cases of chronic abuse, CYP2E1 plays a crucial role in mediating alcohol-induced hepatic and extra-hepatic toxicity. A few recent studies, such as from Kumar's group, have shown that CYP2E1 is abundantly packaged in plasma exosomes in healthy and alcoholic individuals, as well as in rodents [48, 195]. Cho et al. [195], demonstrated that alcohol increased the total number of EVs and CYP2E1 expression in patients with alcoholism and in an alcohol-fed animal model. Interestingly, increased EV production 
and vesicular secretion of several CYP isoforms appears to be modulated by cellular CYP2E1, since these inductive effects were abolished in Cyp2e1-null mice and in the presence of an enzymatic inhibitor, chlormethiazole. Moreover, CYP2E1 activity increased oxidative and endoplasmic reticulum stress, contributing to further vesicular packaging of CYP2E1. These EV-CYP2E1 could potentially act as a biomarker for liver damage from long term alcohol exposure [195]. Kumar et al. [48] observed that the level of CYP2E1 in exosomes derived from healthy human plasma was significantly higher than other CYP isoforms such as CYPs 1B1, 2A6, and 3A4. Most importantly, the exosomal CYP2E1 was metabolically active, which indicates its potential role in contributing to ethanol metabolism and associated oxidative stress and toxicity in extrahepatic regions such as the brain. The major source of this exosomal CYP2E1 is likely to be liver cells, since the levels of CYP2E1 mRNA in the plasma exosomes were reflective of those of hepatocytes. In our own study, when plasma exosomes enriched in CYP2E1 were treated to naïve hepatocytes, they significantly increased alcohol- and acetaminophen-induced toxicity, which were rescued by treatment with a selective CYP2E1 inhibitor [unpublished observations]. This indicates that plasma exosomal CYP2E1 has the potential to contribute to the pathophysiology in recipient cells. Further investigation is ongoing to determine whether these exosomal CYPs, especially CYP2E1, have any role in enhancing alcohol-induced comorbidities.

\section{Potential Mechanisms of Alcohol-induced Toxicity via EVs/exosomes}

Clearly, there is much that remains to be done to fill in the gaps in our current understanding of how alcohol-modified EVs/exosomes may damage extra-hepatic tissues. For example, as of yet, there are no research publications concerning the effects of ethanol on the protein or RNA contents of EVs/exosomes derived from the cells of the central nervous system. It is a mostly untapped area of study, and as such discussion of pathogenic ethanol-induced exosomal content changes is necessarily speculative. Nevertheless, there are some predictions that can be made with confidence, based upon knowledge of overlapping interactions between ethanol exposure and EV/exosomal secretion of established toxic proteins.

\section{Cathepsin enzymes}

Cathepsins are a family of lysosomal proteases that are highly expressed in many cell types, especially phagocytes such as macrophages and microglia. Under stress conditions, cathepsin enzymes (in particular cathepsins B and D) can be secreted into extracellular space, where they can cause neurotoxicity through dysregulated proteolysis [196]. For example, Amritaj et al. [197] demonstrated that the compound U18666A, which is used as an agent for inhibiting cholesterol synthesis and transport in an in vitro model of Niemann-Pick type $\mathrm{C}$ disease, induced expression and extracellular secretion of cathepsin D from hippocampal neurons, which contributed to cell death in neurons and fibroblasts. Fan and He [198] found that astrocytes expressing HIV Tat protein exocytose their lysosomes, releasing cathepsin B and causing neuronal death. 
Both cathepsin B and cathepsin D have been investigated in Alzheimer's disease pathology, as they both are known to process amyloid precursor protein, the pro-peptide of beta amyloid [199, 200] and have been observed to be induced in the brains of Alzheimer's disease rodent models [201, 202] and human patients [203]. There is some evidence that these induced cathepsins may be neuroprotective in that they help to digest beta amyloid plaques [204, 205]. However, CTSB inhibition has also been shown by Hook et al. [206] to reduce beta amyloid and improve memory in a murine Alzheimer's model, indicating that it may also contribute to neurotoxicity. Regardless of this conflicting data on their net effect, the general consensus in the literature is that extracellular secretion of cathepsins increases in cases of Alzheimer's disease [207] and that secreted cathepsins directly cause neuronal apoptosis [197, 208, 209]. Ethanol has been shown to enhance the expression and activity of both cathepsin enzymes in multiple tissues, including the liver [210], pancreas [211], and brain [212]. The release of cathepsin enzymes likely occurs through lysosomal leakage caused by ethanol-induced oxidative stress, as high quantities of ROS can cause membrane instability of that organelle in many cell types [213, 214]. Goetzl et al. [215] observed that cathepsin D was packaged in neuron-derived exosomes secreted into plasma, and that the exosomal expression of cathepsin D was greater in patients with severe Alzheimer's disease. Kang et al. [216] similarly observed increased expression of cathepsin D in exosomes derived from retinal epithelia found in the aqueous humor of patients with macular degeneration. Cathepsins B and D are also secreted in exosomes derived from macrophages in vitro, both of which are dose-dependently induced with ethanol exposure [unpublished observations]. With this in mind, there is good reason to expect that ethanol-induced oxidative stress may induce exosomal secretion of active cathepsin enzymes, which may contribute to long-lasting neurodegenerative conditions.

\section{NADPH oxidase}

Another potential protein of interest is the NADPH oxidase complex (NOX). NOX is expressed in several cell types, particularly phagocytes, and when activated produces large quantities of superoxide, contributing to oxidative stress [217]. The NOX complex has been shown to be both activated and induced by ethanol exposure in neurons, microglia, and macrophages both in vitro and in murine models of alcohol abuse $[218,219]$. Janiszewski et al. [220] were among the first to observe expression of NOX in exosomes and microvesicles in plasma, which was pro-apoptotic when treated to recipient endothelial and aortic smooth muscle cells. While it has never been studied, it is likely that ethanol exposure may induce secretion of EV/exosomal NOX from macrophages and microglia in excess, which could be a potent source of ROS-mediated toxicity. 


\section{Tau}

Drinking alcohol is not definitively associated with disease severity or poorer prognosis in Alzheimer's disease [221], yet oxidative stress is known to have some interaction with the pathways associated with its pathogenesis [222]. As ethanol metabolism is a potent source of ROS there remains potential for alcohol to exacerbate Alzheimer's-associated pathologies. Indeed, it has been observed that ethanol can induce phosphorylation of tau protein, the microtubule-associated protein whose dysfunction is a hallmark of Alzheimer's disease [223, 224]. Ethanol has also been shown by Gendron et al. [225] to induce aggregation of tau in neuroblastoma cells in vitro, and inhibit its clearance. Given that neurons can secrete tau in exosomes [226] and that microglia can propagate the spread of tau through phagocytosis and re-exocytosis [227], it is likely that chronic consumption of alcohol enhances the production and spread of phosphorylated tau aggregates via exosomes, which may contribute to the progression of Alzheimer's disease.

\section{MicroRNA-21}

Alcohol has also been demonstrated to alter the miRNA transcriptome in many tissues, inducing both protective and deleterious effects [228]. However, exosomal transport of ethanol-induced miRNAs has only just begun to be investigated. In particular, there is one miRNA that is known to be induced by ethanol exposure, to be packaged into exosomes, and to have a complex role in the CNS: miR-21. It has long been known that miR-21 has a role in regulating a number of genes, many of which are associated with controlling cellular growth and proliferation. Under conditions of stress, such as ethanol exposure, miR-21 can be induced, balancing tissue regeneration and repair in the liver $[229,230]$. It has also been shown to improve neurological outcomes following traumatic brain injury in rats, indicating potential neuro-regenerative properties as well [231]. However, as mentioned previously [190], these same qualities make miR21 a mediator of cancer in a number of different tissues, as it protects tumor cells by modulating inflammation and apoptosis [232]. It has been observed to be secreted in exosomes in a murine glioblastoma model and elevated in exosomes derived from the serum and spinal fluid of patients with high-grade glioma [233, 234]. In addition to being pro-cancerous, one study by Yelamanchili et al. [235] showed that miR-21 in extracellular vesicles could also be directly neurotoxic through interactions with TLR7. With the knowledge that miR-21 can be induced by alcohol, and given its associations with neurotoxicity and cancer, the scientific community would be well served by greater understanding of the role of exosomal transport of miR-21, and other potentially harmful miRNA that may be induced by ethanol, in these conditions.

\section{HIV elements}

Alcohol consumption is also known to enhance HIV pathogenesis [236-238]. It is very well established that oxidative stress can induce viral replication and the expression 
of viral proteins, by multiple mechanisms [239, 240]. Ethanol metabolism, a significant source of oxidative stress, therefore, also unsurprisingly enhances viral replication in Tcells and mononuclear cells $[236,241]$. This phenomenon also occurs throughout the CNS [242] and consequently alcohol drinking is considered a risk factor for the development of HIV-associated neurocognitive disorders [109, 243]. Aside from inducing viral replication, ethanol also interferes with the metabolism of some antiretroviral drugs, further worsening disease progression in infected alcohol drinkers $[244,245]$. Additionally, ethanol exposure has been shown to potentiate the neurotoxic effects of HIV proteins such as gp120 and Tat, through sensitization of the N-methyl Daspartate receptor, leading to excitotoxic cell death [246, 247].

HIV, like some other viruses, is known to utilize exosome processing and secretion machinery (such as the ESCRT complex) to facilitate its own reproduction [248]. As a consequence of that, viral proteins and RNA are packaged and secreted in exosomes and other extracellular vesicles, even in the presence of antiretroviral therapy [249] These viral elements, such as the proteins Tat and Nef, have been shown to have a variety of pathogenic effects [250-252]. Taken together, it is highly likely that the exosomal transport of HIV components is enhanced by ethanol exposure through oxidative stress-induced transcription of the viral genome, leading to increased susceptibility to intercellular viral infection, inflammation, direct neurotoxicity, and other deleterious conditions associated with HIV infection. However, this phenomenon, like the other previously suggested mechanisms of ethanol-induced toxicity mediated by exosomes, has not yet been demonstrated experimentally, and is merely speculative given the available data. This is an area in great need of additional research, as alcohol abuse and its effects on neurodegeneration are still not yet fully understood but continue to affect millions of people worldwide. Figure 1-2 provides a summarized illustration of the above discourse.

\section{Discussion}

Alcohol alters the miRNA and protein content (e.g. miR-27a, miR-122, CD40L) of EVs such as exosomes from multiple tissues, having complex immunomodulatory effects. EV/exosomal RNA (miR-21, miR-19b, VEGF mRNA) and proteins (HSP90, CCN2) can be potentially involved in alcohol-induced exacerbation of existing disease conditions, such as pancreatic fibrosis, cardiomyopathy, and some cancers. We also considered some potential EV/exosomal agents that may contribute to alcohol-induced damage in other tissues, such as the central nervous system (NOX, cathepsin enzymes), as well as exosomal elements associated with other conditions that may be enhanced by alcohol exposure (phosphorylated tau and HIV protein and RNA elements). In conclusion, recent investigations, limited though they are, point to a consensus that exosomes derived from alcohol-exposed cells, irrespective of origins, can play a crucial role in disease progression by modulating biological pathways via delivery of cargo to recipient cells. This is an exciting area of research which is in need of further investigation. 


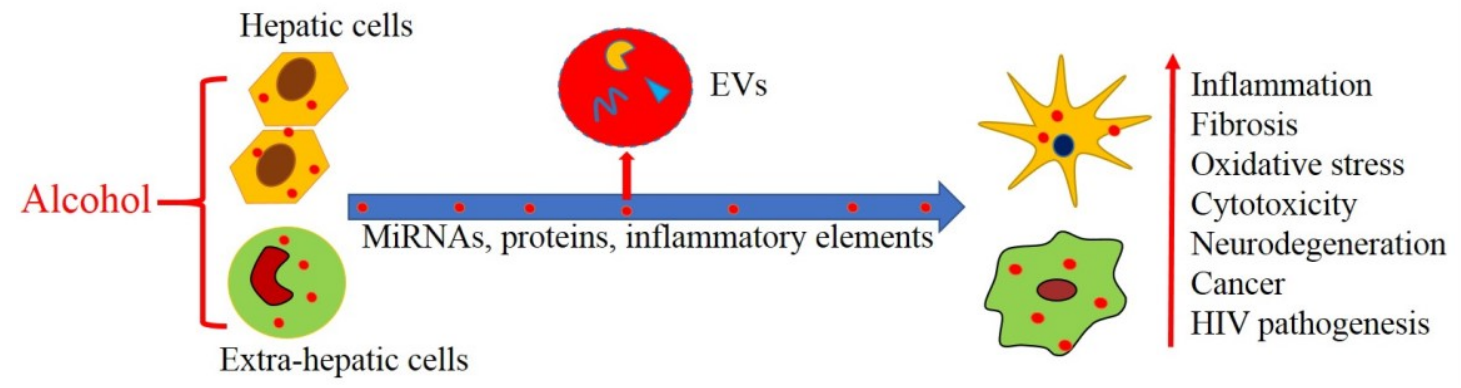

Figure 1-2. Potential EV/exosomal mechanisms of alcohol-induced toxicity.

In response to ethanol exposure, exosomal transport of pathogenic elements to nearby or distant cells can act as cellular messenger system which may facilitate/enhance disease progression in cancer, HIV, liver disease, neurodegenerative disorders, etc. 
EVs/exosome are not just buzzword in the scientific community anymore. As a result of advances in large-scale proteomic analysis since the early 2000 s, research in this area has taken a major leap forward in recent years. However, we are still in the nascent phase of the EV/exosome revolution. In other words, extensive investigation is essential to establish a complete understanding of the role of EVs/exosomes in disease progression, particularly in the context of concurrent xenobiotic exposures. For example, the impact of alcohol on EV contents in alcohol-related co-morbidities is an understudied area, despite its widespread use and well-established role in exacerbating many pathological conditions. Due to limited literature availability and the lack of well-defined target pathways of alcohol-associated co-morbidities, it is apparent that there is a critical need to find biomarkers for alcohol-induced toxicity outside of the liver. Recent reports suggest EVs/exosomes can play a crucial role in finding these desired biomarkers [253]. The circulation of EVs/exosomes throughout the body via body fluids, particularly plasma, urine, and saliva, make them appealing, because of their convenient accessibility for sample collection, sometimes referred to as "liquid biopsy" [254]. There have been several studies identifying EVs with specific contents as biomarkers for various conditions, especially cancers [255]. The ability of EVs/exosomes to migrate from their tissues of origin into wider circulation through plasma also allows for diagnosis of disease in otherwise less accessible regions of the body, such as the CNS. For example, neuronally-derived exosomes collected from plasma have been found to carry markers for Alzheimer's disease in Alzheimer's patients, as well as in individuals with Down syndrome $[215,256]$. It is highly likely that circulating exosomes contain specific markers for alcohol-induced toxicity outside of the liver, which would provide a novel and non-invasive way of diagnosing specific morbidities in chronic alcohol drinkers that require therapeutic intervention. As discussed in this review, in a murine chronic pancreatitis model, exosomes appear to play a crucial role in pancreatic fibrogenesis, a pathological outcome that is enhanced by chronic alcohol exposure [194]. Identification of pancreatic exosomes containing high levels of the fibrogenic elements CCN2 and miR21 in the plasma of chronic drinkers could allow for earlier diagnosis and therapy to counteract alcohol-enhanced pancreatitis and fibrosis. Considering that miR-21 is also induced in the liver during alcohol metabolism, and is furthermore a known oncogenic agent, miR-21 and other EV/exosomal ethanol-induced miRNAs may be valuable early indicators of alcohol-associated cancers [229, 230, 232-234, 257]. Table 1-1 contains the list of pathogenic elements that are involved in ALC-induced disease pathogenesis.

Based on the literature reports, it is evident that alcohol-induced changes in cellular contents are also coincided with the changes in their EV/exosomal cargos. Alcohol exposure modifies the contents of both hepatic and extra-hepatic EVs which are translocated to other naïve liver cells or non-hepatic cells. This intercellular transfer of biomolecules results in increased/decreased inflammatory responses in the recipient cells. EVs, especially exosomes represent the next frontier in the areas of diagnosis and therapy of a plethora of pathological conditions, many of which are enhanced or altered by concurrent substance abuse. It is vital for patient care that the role of EVs/exosomes in these conditions, and how that role changes in cases of chronic drug use, is elucidated. 
Table 1-1. Pathogenic exosomal elements in response to alcohol exposure.

\begin{tabular}{|c|c|c|c|c|}
\hline Donor cells & Recipient cells & Mediators & Implications & References \\
\hline Hepatocytes & Monocytes & miR-122 & $\begin{array}{l}\text { Immune } \\
\text { sensitization in } \\
\mathrm{AH}\end{array}$ & {$[175]$} \\
\hline Hepatocytes & Macrophages & CD40L & $\begin{array}{l}\text { Macrophage } \\
\text { activation in ALD }\end{array}$ & {$[177]$} \\
\hline Hepatocytes & $\begin{array}{l}\text { Hepatocytes, } \\
\text { monocytes }\end{array}$ & Hsp90 & $\begin{array}{l}\text { Macrophage } \\
\text { activation in ALD } \\
\text { mice }\end{array}$ & {$[178]$} \\
\hline Hepatocytes & Hepatocytes & $\begin{array}{l}\text { Ceramidase and } \\
\text { sphingosine } \\
\text { kinase- } 2\end{array}$ & Fibrogenesis & [181] \\
\hline $\begin{array}{l}\text { Hepatic stellate } \\
\text { cells }\end{array}$ & $\begin{array}{l}\text { Hepatic stellate } \\
\text { cells }\end{array}$ & $\operatorname{miR}-19 b$ & $\begin{array}{l}\text { Increased hepatic } \\
\text { fibrogenesis }\end{array}$ & [184] \\
\hline Monocytes & Monocytes & mi-R27a & $\begin{array}{l}\text { Differentiation to } \\
\text { M2 macrophages }\end{array}$ & {$[185]$} \\
\hline Endothelial cells & Endothelial cells & $\begin{array}{l}\text { MALAT1 and } \\
\text { HOTAIR }\end{array}$ & $\begin{array}{l}\text { CD34-mediated } \\
\text { vascularization }\end{array}$ & [188] \\
\hline $\begin{array}{l}\text { Retinal pigment } \\
\text { epithelium cells }\end{array}$ & Endothelial cells & $\begin{array}{l}\text { VEGFR-1 and } \\
\text { VEGFR-2 }\end{array}$ & Vascularization & [189] \\
\hline $\begin{array}{l}\text { Oral squamous } \\
\text { cell carcinoma }\end{array}$ & Monocytes & $\operatorname{miR}-21$ & $\begin{array}{l}\text { NF-kB activation } \\
\text { and } \mathrm{MCP}-1 \\
\text { secretion }\end{array}$ & [190] \\
\hline $\begin{array}{l}\text { Pancreatic stellate } \\
\text { cells }\end{array}$ & $\begin{array}{l}\text { Pancreatic stellate } \\
\text { cells }\end{array}$ & $\begin{array}{l}\text { CCN2 and } \\
\text { miR-21 }\end{array}$ & $\begin{array}{l}\text { Collagen } \\
\text { production and } \\
\text { fibrogenesis }\end{array}$ & [194] \\
\hline
\end{tabular}


A more complete understanding of the interplay between EVs/exosomes and alcohol will allow for greater degrees of personalized care for the individuals who consume it or other commonly used substances such as tobacco constituents, cannabinoids, and opioids, ultimately leading to superior public health outcomes.

Based on the scientific discourse above, we undertook two unique projects to 1) identify a superior alternative to DAS as a CYP2E1 inhibitor which can be used in chronic mechanistic studies related to CYP2E1 mediated toxicity and 2) to investigate the potential role of exosomal CYP2E1 in mediating xenobiotic-induced toxicity. The principal component of these two projects is xenobiotic- and diseases-induced CYP2E1associated toxicity.

\section{Project 1}

\section{Scientific premise}

The scientific premise of the first project is based on the literature reports: 1) CYP2E1 is involved in metabolic activation of several clinically important xenobiotics such as alcohol, acetaminophen, etc. [1, 40, 56, 79, 258]; 2) CYP2E1 is induced by its own substrates and hence, can cause further oxidative stress and acetaldehyde production which can lead to severe hepatic and extra-hepatic toxicity [259-261]; 3) Diallyl sulfide (DAS) is a selective inhibitor of CYP2E1 and has been shown to have protective effects in xenobiotics- and diseased-induced toxicity in many in vitro and in vivo studies [120]; 4) DAS is also a substrate of CYP2E1, and its metabolism by CYP2E1 produces toxic sulfur-containing metabolites [120]; 5) Due to its own toxicity at relatively high concentrations and in cases of chronic use, the usage of DAS is limited mainly to acute study; 6) Molecular docking study has been identified as a tool for the identification of potentially novel compounds as enzyme inhibitors [262-265].

\section{Hypothesis}

Based on these scientific premises, we hypothesize that structural modification with the aid of molecular modeling improves the efficacy and safety profile of novel DAS analogs. The new analogs are less cytotoxic than DAS at high concentrations and upon chronic exposure and are capable of reducing CYP2E1-mediated toxicity in hepatic and extra-hepatic cells especially in alcohol and acetaminophen abuse.

\section{Objective and aims}

The objective of the project is to identify relatively safer and more potent DAS analogs which can be used, at least, as a suitable research tool in CYP2E1-related study design under chronic condition. 


\section{Aim 1: Kinetic characterization of DAS analogs for their novel role as CYP2E1 inhibitors}

- Molecular docking assay

- CYP2E1 inhibition assay

- CYP2E1 kinetic data analysis

\section{Aim 2: Evaluation of structural analogs of DAS as novel CYP2E1 inhibitors for their protective effect against xenobiotic-induced toxicity and HIV replication}

- Cell culture (hepatic, monocytic, and astrocytic cell lines) and treatment

- CYP2E1 activity in U937 monocytic and SVGA astrocytic cells

- Measurement of reactive oxygen species and cell viability using flow cytometry

- XTT cell viability assay

- LDH activity assay

- Caspase 3 activity assay

- HIV p24 ELISA

- Statistical analysis

\section{Project 2}

\section{Scientific premise}

The scientific premise of this project is based on the available literature reports: 1) Exosomes, cellular nanovesicles $(<150 \mathrm{~nm})$, are secreted by almost all cell types into their extra-cellular environment and biofluids such as plasma; 2 ) The semi-selective ability of exosomes to package and transport diverse biological cargos such as proteins, mRNA, miRNA, and small molecules allow them to serve both as diagnostic biomarkers of disease states and as potential therapeutic targets [175, 266];3) Exosomal transport between cells via plasma can be either beneficial or deleterious [267-269]; 4) It has been recently reported that a relatively large amount of functional CYP2E1 is present in human plasma exosomes compared to other CYP enzymes [48]; 5) Study has also shown that the relative levels of CYP2E1 mRNA and protein in plasma exosomes are higher than those of monocytic cells and exosomes derived from hepatic and monocytic cells [48]; 6) Upon exposure to naïve cells, exosomal content have been shown to alter the pathophysiology of the recipient cells [166]; 7) Although, CYP2E1 mediated hepatic toxicity is well characterized, the mechanism of extra-hepatic toxicity by CYP2E1 mediated xenobiotic (e.g. alcohol, acetaminophen, etc.) metabolism is not fully understood $[40,270]$. 


\section{Hypothesis}

Based on these scientific premises, we hypothesize that plasma exosomes contribute to alcohol- and acetaminophen-induced toxicity by delivering its CYP2E1 cargo into the naïve recipient cells.

\section{Objective and aim}

The objective of the project is to evaluate the role of plasma exosomal CYP2E1 in exacerbating alcohol- and acetaminophen-induced toxicity in hepatic and extra-hepatic cells.

Aim: Investigation of the role of plasma exosomal CYP2E1 in alcohol- and acetaminophen-induced toxicity

- Cell culture and treatment

- Isolation and validation of exosomes from human and mouse plasma

- Acetylcholine esterase assay

- Exosomes labeling and uptake

- CYP2E1 SiRNA transfection and treatment

- Western blot

- LDH cell viability assay

- Statistical analysis 


\section{CHAPTER 2. KINETIC CHARACTERIZATIONS OF DIALLYL SULFIDE ANALOGS FOR THEIR NOVEL ROLE AS CYP2E1 ENZYME INHIBITORS ${ }^{2}$}

\section{Introduction}

Diallyl sulfide (DAS), a thioether present in garlic extract, has garnered significant attention from scientific communities since the early 1990s. Researchers have explored the use of DAS for its multifarious applications that includes anti-cancer, antioxidant, and anti-inflammatory properties [120]. DAS has been used most frequently in the prevention against toxicities induced by xenobiotics such as alcohol and acetaminophen by selectively inhibiting cytochrome P450 2E1 (CYP2E1) [120]. CYP2E1 is involved in the metabolism of more than 85 xenobiotics [92]. CYP2E1mediated metabolism produces reactive oxygen species (ROS) and toxic metabolites, which causes cytotoxicity. For example, CYP2E1 metabolism of acetaminophen (APAP) causes production of a toxic metabolite N-acetyl-p-benzoquinone imine, and APAPinduced liver injury accounts for more than half of overdose-related liver failure in the United States [271]. In addition, ethanol is known to induce CYP2E1, which subsequently metabolizes ethanol and causes ROS and acetaldehyde mediated cellular toxicity and cancer of hepatic and non-hepatic organs/systems [39, 272]. DAS, through CYP2E1 inhibition, has shown protective effects against ethanol- and APAP-mediated hepatoxicities in several studies [273-275]. DAS has also been extensively used both in vitro and in vivo study as a tool to inhibit CYP2E1 in hepatic as well as a number of extra-hepatic cells [276].

However, despite DAS being a selective inhibitor of CYP2E1, it has failed to achieve clinical relevance as a drug, as well as, its application in chronic in vitro/vivo study as a tool due to its own potential toxicity. At a relatively high concentration and chronic exposure, DAS is toxic to hepatic and extra-hepatic cells [120]. In addition, although DAS is being used as a tool to inhibit CYP2E1, it is poorly characterized with regards to CYP2E1 inhibition. Our major goal is to find a superior alternative to DAS, which overcomes the limitations of DAS, and can be used as a better research tool to inhibit CYP2E1 in various pathological conditions. Furthermore, novel DAS analogs have the potential to be used as adjuvant therapy in various pathological conditions. In this study, we selected seven commercially available compounds, which resembles DAS structure. These compounds were characterized for binding affinity using ligand-docking analysis followed by CYP2E1 inhibition study.

\footnotetext{
${ }^{2}$ Reprinted from final submission with open access permission from John Wiley and Sons. Rahman M. A., Midde N. M., Wu X., Li W., and Kumar S. Kinetic Characterizations of Diallyl Sulfide Analogs for Their Novel Role as CYP2E1 Enzyme Inhibitors (2017). Pharmacol Res Perspect. Oct, 5(5). doi: $10.1002 / \operatorname{prp} 2.362$.
} 


\section{Materials and Methods}

\section{Chemicals}

Diallyl sulfide (DAS), allyl methyl sulfide (AMS), allyl ethyl sulfide (AES), diallyl ether (DE), thiophene (TP), 2-(prop-2-en-1-yloxy) ethan-1-amine (PEA), and 5hexen-1-amine (5,1 HA) were purchased from Sigma-Aldrich (St. Louis, MO). 2-prop-2enoxyacetamide (PEXA) was purchased from Aldlab Chemicals (Woburn, MA). The stock solutions for these compounds were prepared in acetonitrile that was bought from Sigma.

\section{Molecular docking study}

Molecular modeling studies were performed using the Schrodinger Molecular Modeling Suite 2015 (Schrödinger, LLC, NY) as described [277, 278]. The initial models of human CYP2E1 for docking were taken from the Protein Data Bank. Among the six $\mathrm{X}$-ray crystal structures available for human CYP2E1, we chose the $3 \mathrm{KOH}$ for docking DAS and all the analogs based on completeness, resolution, and ligand binding pose [279]. Protein and grid preparations were performed using the Protein Preparation Wizard with default protocol, and the grid of the CYP2E1 active site containing heme iron was defined. All compounds were prepared using the Ligprep module, before they were docked into the active site with metal constraints using the Glide module in the software.

\section{CYP2E1 inhibition assay}

The inhibition of CYP2E1 activity by DAS or its analogs was measured using standard para-nitrophenol (p-NP) hydroxylation assay as described before [280]. Briefly, the final reaction mixture contained $50 \mathrm{mM}$ potassium phosphate buffer $(\mathrm{pH} 7.4), 2$ $\mathrm{pmol} / \mu \mathrm{L}$ of CYP2E1 baculosomes (Thermo Fisher Scientific, Waltham, MA), and varying concentration of $\mathrm{p}-\mathrm{NP}(5,15,30,50$, and $100 \mu \mathrm{M})$ and inhibitors $(5,10,25,50$, 100 , and $200 \mu \mathrm{M}$ ). Upon pre-incubation at $37^{\circ} \mathrm{C}$ for 5 minutes, the reaction was initiated by adding $26 \mathrm{mM} \mathrm{NADPH}$ and carried out for an hour at $37^{\circ} \mathrm{C}$. Upon terminating the reaction by $20 \%(\mathrm{w} / \mathrm{v})$ trichloroacetic acid, the reaction was neutralized by $2 \mathrm{M} \mathrm{NaOH}$ and absorbance was measured at $535 \mathrm{~nm}$ using a micro plate reader (Cytation ${ }^{\mathrm{TM}} 5$ Cell Imaging Multi-Mode Reader, BioTek, VT). The absorbance without an inhibitor was normalized to $100 \%$ activity of CYP2E1.

\section{CYP2E1 kinetic data analysis}

The CYP2E1 inhibition kinetics were analyzed using GraphPad Prism 5 software (GraphPad Software Inc., San Diego, CA). The half maximal inhibitory concentration ( $\mathrm{IC}_{50}$ ) was determined by fitting curve to data by non-linear regression. The Michaelis- 
Menten constant $\left(\mathrm{K}_{\mathrm{m}}\right)$ and maximal reaction velocity $\left(\mathrm{V}_{\max }\right)$ were obtained by using the Michaelis-Menten non-linear regression equation. The experimental data were reanalyzed with the appropriate equation to determine the inhibition type and inhibition constant $\left(\mathrm{K}_{\mathrm{i}}\right)$. Goodness-of-fit criteria were used to define the inhibition pattern.

\section{Results and Discussion}

\section{Docking study of the analogs with CYP2E1 active site}

To find a superior DAS alternative, we performed a structure activity study. The central sulfur atom in DAS structure interacts with the heme of CYP2E1 active site, thereby acting as an inhibitor. However, DAS is also metabolized at the same position resulting in production of toxic sulfur metabolites such as diallyl sulfoxide and diallyl sulfone [120]. We analyzed seven compounds (AMS, AES, DE, TP, PEA, 5,1 HA, and PEXA) that resemble DAS, but have slightly different chemical moieties (Figure 2-1A). We chose analogs that altered DAS at the central hetero atom by replacing sulfur with oxygen (DE, PEA, and PEXA), or by completely removing sulfur (5,1 HA). These alterations should result in DAS analogs retaining their ability to interact with the heme of CYP2E1 active site but losing their ability to be oxidized into toxic metabolites. We also chose DAS analogs that had stronger nucleophiles at the carbon atom adjacent to the hetero atom (PEA, PEXA, and 5,1 HA) to increase the binding strength of the analogs to the active site. Furthermore, we chose compounds with smaller side chain (AES and AMS), which is likely to improve the binding affinity as well [120]. Low molecular monocyclic aromatic compounds are also a viable option as the binding site of CYP2E1 (Figure 2-1B) exists within a narrow channel, unlike other CYP active sites [281]. Therefore, we chose a cyclic analog of DAS (TP), which is likely to strengthen the binding as an inhibitor.

To determine the potential binding mode and molecular interactions between the analogs and CYP2E1 at the active site, we performed a docking study. All the compounds generally showed similar binding mode, in that they occupied approximately the same space surrounded by a group of hydrophobic residues and the "necklace" of phenylalanine side chains including Phe298, Phe116, and Phe207, above the heme (Figure 2-1B). In each structure, the heteroatom (oxygen or sulfur) interacts with the heme iron with distances of $3.15-3.58 \AA$, whereas the alkyl chain or aromatic ring extends towards the helix I. Among all the compounds, TP favors a $\pi-\pi$ interaction with the phenylalanine side and shows the best docking score of -4.86 (Table 2-1). However, unlike the thiophene ring, the alkyl chains in the other compounds are flexible and lack a $\pi-\pi$ interaction with the surrounding residues. Therefore, the study predicts that introducing aromatic moieties would increase interactions with CYP2E1. We also observed a higher docking score for AMS (-3.24) than DAS (-1.56). These results are consistent with our hypothesis. 
(A)<smiles>C=CCSCC=CC(CC(C)(C)C)C(=O)OCC</smiles><smiles>C=CCOCC=C</smiles>

Diallyl ether (DE)

(B)

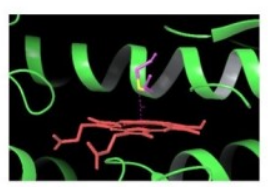

DAS

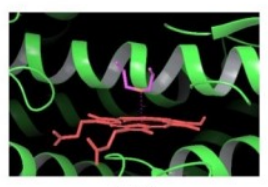

DE
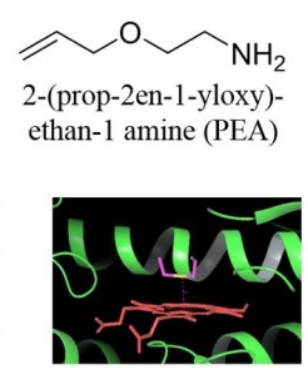

AES

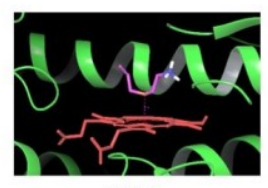

PEA
$\mathrm{N}_{\mathrm{S}}$

Allyl methyl sulfide (AMS)
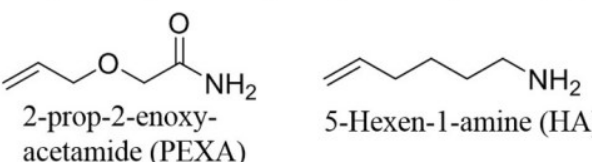

5-Hexen-1-amine (HA)

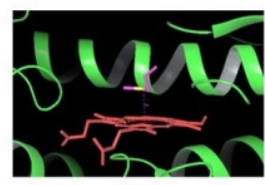

AMS

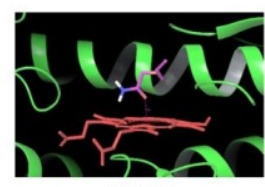

PEXA

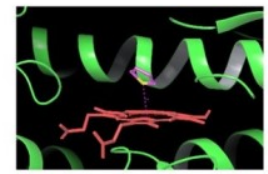

TP

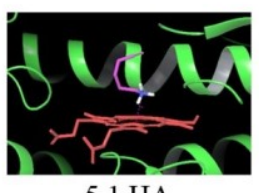

5,1 HA

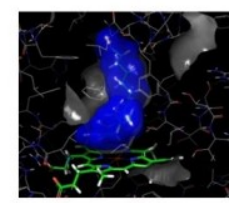

CYP2E1 active site

Figure 2-1. Structure of the analogs and docking with the CYP2E1 crystal structure.

A) The structures of the seven commercially available DAS analogs that were used for this study. B) Docking of DAS and analogs with human CYP2E1 crystal structures using Schrödinger Molecular Modeling Suite 2015. All the compounds were prepared using the Ligprep module, and then docked into the active site with metal constraints. The results showed similar binding mode for all DAS analogs 
Table 2-1. Summary of the CYP2E1 inhibition kinetics of the analogs.

\begin{tabular}{|c|c|c|c|c|c|}
\hline Analogs & Inhibition type & $\mathbf{K}_{\mathbf{i}}(\boldsymbol{\mu} \mathbf{M})$ & $\mathrm{IC}_{50}(\boldsymbol{\mu M})$ & $\begin{array}{c}\text { Maximal } \\
\text { inhibition }(\%)\end{array}$ & $\begin{array}{c}\text { Docking } \\
\text { score }\end{array}$ \\
\hline $\begin{array}{l}\text { Diallyl sulfide } \\
\text { (DAS) }\end{array}$ & Competitive & $6.3 \pm 1.0$ & $17.3 \pm 1.1$ & 97 & -1.54 \\
\hline $\begin{array}{l}\text { Allyl methyl } \\
\text { sulfide (AMS) }\end{array}$ & Competitive & $4.4 \pm 0.9$ & $11.4 \pm 1.1 *$ & 100 & -3.25 \\
\hline Thiophene (TP) & Competitive & $7.7 \pm 1.2$ & $19.4 \pm 1.1$ & 98 & -4.86 \\
\hline $\begin{array}{l}\text { Diallyl ether } \\
\text { (DE) }\end{array}$ & Competitive & $3.1 \pm 0.2^{*}$ & $6.3 \pm 1.2^{*}$ & 100 & -0.17 \\
\hline \multirow{2}{*}{$\begin{array}{l}\text { 5-hexen-1-amine } \\
(5,1 \mathrm{HA})\end{array}$} & Uncompetitive & $\sim 7.3$ (ambiguous) & \multirow[t]{2}{*}{$74.7 \pm 1.2^{*}$} & \multirow[t]{2}{*}{75} & \multirow[t]{2}{*}{-1.88} \\
\hline & Non-competitive & $\sim 10.5$ (ambiguous) & & & \\
\hline \multirow{4}{*}{$\begin{array}{l}\text { 2-(prop-2-en-1- } \\
\text { yloxy) ethan-1- } \\
\text { amine (PEA) } \\
\text { 2-prop-2- } \\
\text { enoxyacetamide } \\
\text { (PEXA) }\end{array}$} & Uncompetitive & $19.3 \pm 2.3 *$ & \multirow[t]{2}{*}{$43.7 \pm 1.4^{*}$} & \multirow[t]{2}{*}{71} & \multirow[t]{2}{*}{-1.27} \\
\hline & Non-competitive & $34.1 \pm 3.3 *$ & & & \\
\hline & Uncompetitive & $246 \pm 47 *$ & \multirow[t]{2}{*}{$91.4 \pm 1.6^{*}$} & \multirow[t]{2}{*}{20} & \multirow[t]{2}{*}{-1.73} \\
\hline & Non-competitive & $363 \pm 64 *$ & & & \\
\hline
\end{tabular}


Docking score does not always depict ligand-receptor interactions accurately. In fact, the flaw of the scoring system is considered to be a major limiting factor of computational docking [282], especially for small ligands for which the number of interactions between a ligand and its receptor is too small to make reliable differentiations among different ligands. The docking programs make simplified assumptions in the assessment of modelled complexes without accounting for physical phenomena that play a role in influencing molecular recognition, such as entropic effects [283]. For example, replacing the sulfur atom of the DAS structure with oxygen (DE) should have resulted in similar or increased binding affinity. However, interestingly, we observed lowest docking score for DE, when we would have expected this compound to be at the least equally potent to DAS in its activity. Moreover, we expected increased binding for the compounds in which modification have been made at the carbon atom adjacent to the heteroatom by integrating stronger nucleophiles (e.g. PEXA). However, we did not observe much increase in docking score for such analogs. Nevertheless, this preliminary docking study provided some rough comparisons among the chosen ligands.

\section{Inhibition of CYP2E1 activity by DAS and its analogs}

There is very little information about the precise CYP2E1 inhibition capacity of DAS. In a few studies, DAS has been shown to be a competitive inhibitor of CYP2E1 using human and rat liver microsomes [143, 284]. However, these studies were performed either with insufficient or very low concentrations of DAS. In these studies, the reported $\mathrm{K}_{\mathrm{i}}$ values of DAS therefore differ by a large margin, perhaps due to the lack of sufficiently varying degree of concentrations of both the inhibitor as well as the substrate. The study by Morris et al used a couple of substrate and inhibitor concentrations and provided a better inhibition kinetic profile. Moreover, at first, we characterized the DAS inhibition kinetics with CYP2E1 using multiple concentrations of both p-NP and DAS (Figure 2-2, Table 2-1).

We used CYP2E1 baculosomes for our study since they have the unique property of expressing only one type of CYP isozyme. This gives an advantage over traditional microsome-based assays in that there will be no interference/interactive effects from other CYPs in determining the inhibition kinetics. First, we established that CYP2E1 activity is linear at $100 \mu \mathrm{M}$ substrate concentration for one hour of reaction (data not shown). We then conducted the inhibition assay at five different $p$-NP concentrations (5, $15,30,50,100 \mu \mathrm{M})$ and six different concentrations of inhibitors $(5,10,25,50,100$, and $200 \mu \mathrm{M})$. Based on the reported $\mathrm{K}_{\mathrm{m}}$ value, $100 \mu \mathrm{M}$ may not be the saturating $\mathrm{p}-\mathrm{NP}$ concentration. However, CYP2E1 shows substrate inhibition at $>100 \mu \mathrm{M}$ p-NP [281]. Therefore, first we performed the inhibition assay at $100 \mu \mathrm{M}$ to obtain the $\mathrm{IC}_{50}$ values (Table 2-1). Subsequently, we used lower concentrations of the substrate to determine the $\mathrm{IC}_{50}$ at each substrate concentration as well as the $\mathrm{K}_{\mathrm{i}}$ values. Our results showed that DAS has an $\mathrm{IC}_{50}$ of $17.3 \pm 1.1 \mu \mathrm{M}$ and a $\mathrm{K}_{\mathrm{i}}$ of $6.3 \pm 1.0 \mu \mathrm{M}$, and it follows competitive inhibition kinetics. Brady et al. used acetone-induced rat microsome for their study and only one concentration of DAS $(125 \mu \mathrm{M})$, and they observed that inhibition of CYP2E1 by DAS followed competitive inhibition, which is consistent with our findings 

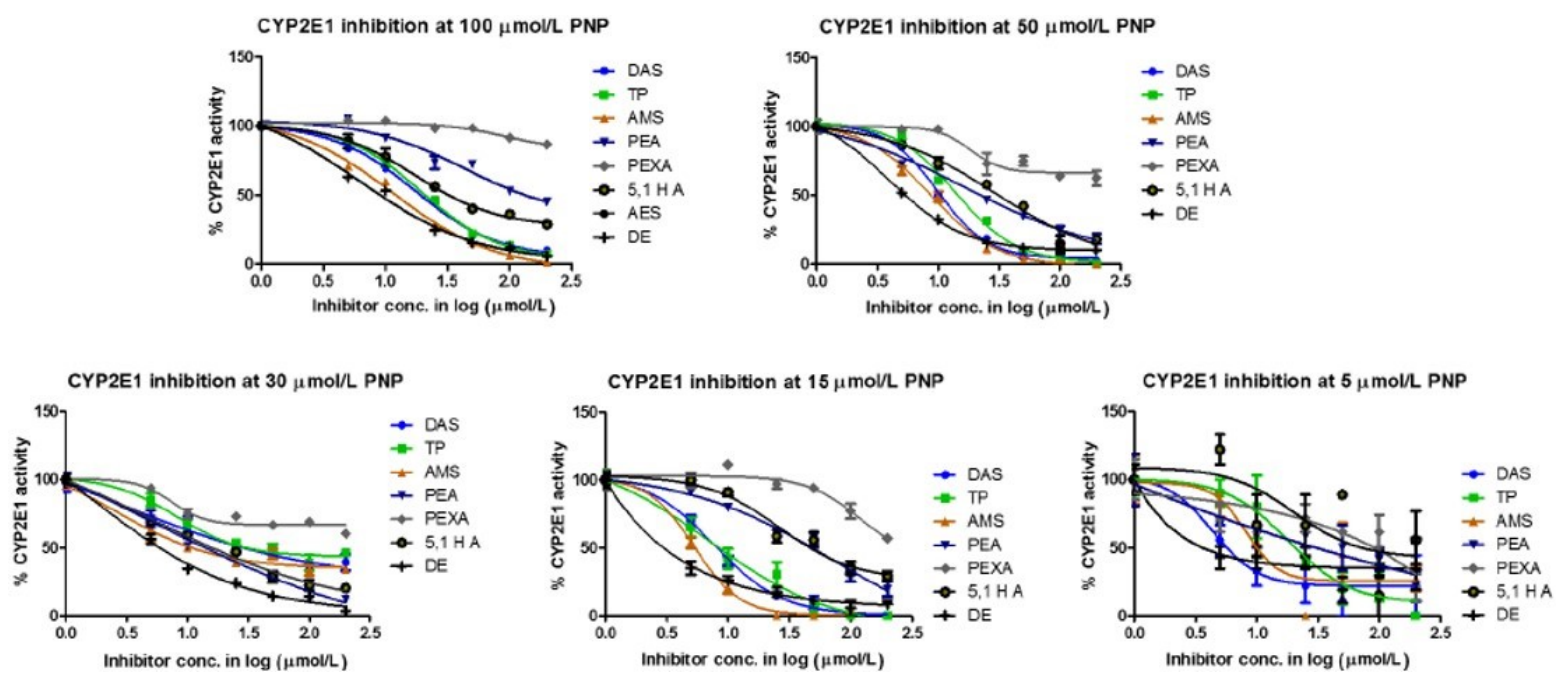

Figure 2-2. Inhibition of CYP2E1 activity at varying p-NP substrate and inhibitor concentrations.

The absorbance obtained without any inhibitor (vehicle only) was considered as $100 \%$ CYP2E1 activity for each substrate concentration. The data was analyzed using nonlinear regression curve fitting. The $\mathrm{IC}_{50}$ was determined and presented in Table 2-1. The data was also used to plot Michaelis-Menten kinetics curve and $\mathrm{Ki}$ as well as type of inhibition were determined as described in Materials and Method. 
[143]. However, the reported $\mathrm{K}_{\mathrm{i}}$ value $(188 \mu \mathrm{M})$ from their study varies by a large margin from our results as well as from the study conducted by another group using human and rat microsome. Morris et al. reported an $\mathrm{IC}_{50}$ value of $3 \mu \mathrm{M}$ for DAS. However, they used only two lower concentrations $(2$ and $8 \mu \mathrm{M})$ [284]. The reported $\mathrm{K}_{\mathrm{i}}$ values $(0.3 \sim 1.9$ $\mu \mathrm{M})$ were also determined using limited concentrations of both DAS $(0.25$ and $1.0 \mu \mathrm{M}$ for human CYP2E1; 2 and $6 \mu \mathrm{M}$ for rat CYP2E1) and the substrate. Our results are likely to provide more accurate and reliable $\mathrm{IC}_{50}$ and $\mathrm{K}_{\mathrm{i}}$ than the reported values, because we used multiple substrate and DAS concentrations and used CYP2E1-induced baculosomes.

Furthermore, we performed a complete inhibition kinetics of seven DAS analogs as described above (Figure 2-2, Table 2-1). Of the seven compounds, although AES caused marked inhibition, it did not fit with any inhibition kinetics. Therefore, in subsequent studies, we used only six compounds for complete inhibition kinetic analysis. Results show that, contrary to the docking analysis, DE has significantly lower $\mathrm{IC}_{50}$ and $\mathrm{K}_{\mathrm{i}}$ values $\left(6.3 \pm 1.2^{*} \mu \mathrm{M}\right.$ and $3.1 \pm 0.2^{*} \mu \mathrm{M}$, respectively) than DAS $(17.3 \pm 1.1 \mu \mathrm{M}$ and $6.3 \pm 1.0 \mu \mathrm{M}$, respectively). The analog with the highest docking score, TP, showed the similar inhibitory capacity as compared to DAS $\left(\mathrm{IC}_{50}=19.4 \pm 1.1 \mu \mathrm{M}\right.$ and $\mathrm{K}_{\mathrm{i}}=7.7 \pm 1.2$ $\mu \mathrm{M})$. AMS, which exhibited relatively higher docking score, showed an $\mathrm{IC}_{50}$ of $11.42 \pm$ $1.1^{*} \mu \mathrm{M}$ and $\mathrm{K}_{\mathrm{i}}$ of $4.4 \pm 0.9 \mu \mathrm{M}$, which are lower than DAS. Similar to DAS, these analogs showed competitive inhibition of the CYP2E1 activity. Thus AMS, TP, and DE showed complete inhibition at saturating substrate concentration (Table 2-1), suggesting that their nature of inhibition for CYP2E1 is similar to DAS. Other analogs- AES, 5,1 HA, PEA, and PEXA, despite having similar binding conformation and score, did not exhibit desirable inhibitory activity and mostly followed un-competitive and/or noncompetitive inhibition kinetics.

As expected, the replacement of oxygen and/or changing the size of side chain of DAS improved the binding of oxygen or sulfur with the heme of CYP2E1 and showed better inhibition profiles with DE and AES than DAS. This mode of binding is expected to produce metabolite by CYP2E1, which may be less toxic to the metabolites produced by the oxidation of sulfur. It's interesting to note that compounds that showed higher than or similar to DAS binding affinity $\left(\mathrm{IC}_{50}\right.$ or $\mathrm{K}_{\mathrm{i}}$ ) also showed similar inhibition kinetics to DAS. However, the compounds that showed lower binding affinity than DAS showed very different inhibition kinetics. These compounds appear to bind the CYP2E1 active site in two different modes; one through oxygen and the other through nitrogen. The two binding modes appeared to have weaken the overall binding affinity. We had initially expected that nitrogen moieties on PEA, PEXA, and 5,1 HA is likely to strengthen the binding of the heme of CYP2E1.

\section{Conclusion}

These results are significant in terms of finding a superior alternative to DAS. Despite having substantial pharmacological activities and being used in numerous research studies for many years, DAS has failed to consolidate its credibility to be a potential drug candidate [120]. Due to its own toxicity, it is difficult to design any long 
term/chronic treatment schedule with DAS. The metabolites, diallyl sulfoxide and diallyl sulfone, by undergoing further metabolic activation to their respective epoxides can cause considerable toxicity. Moreover, these metabolites can cause autocatalytic destruction of the CYP2E1 enzyme [120]. Zhai et al. has reported that DAS aggravated the isoniazid induced toxic effect by potentiating increased ROS production in primary rat hepatocytes [285]. Our own group have found that despite rescuing ethanol- mediated cell death to a certain extent, DAS itself causes $\sim 15 \%$ cell death in SVGA astrocytic cell lines in 24 hours [115]. The preliminary results (unpublished observations) with DAS analogs showed that, even at as high as $200 \mu \mathrm{M}$ concentrations, TP and DE did not show any significant toxicities for six and four days of treatment, respectively. However, as expected, DAS caused more than $40 \%$ cell death at $200 \mu \mathrm{M}$ after two days of treatment. In conclusions, this is the first report on a thorough analysis of CYP2E1 inhibition kinetics of DAS and its seven structural analogs. This study provided two DAS analogs that have better inhibition characteristics than DAS, which also appear to show relatively lower toxicity than DAS in early studies. Our extensive toxicity profile study of these compounds, and whether these compounds can rescue alcohol- and acetaminophenmediated toxicity in hepatic and extra-hepatic cells is underway. Overall the results are very encouraging in the search for a superior alternative to the widely used DAS as a selective CYP2E1 inhibitor as a research tool and/or as a possible combination/preventive therapy in alcohol- and other xenobiotic-mediated toxicity. 


\section{CHAPTER 3. IN VITRO EVALUATION OF STRUCTURAL ANALOGS OF DIALLYL SULFIDE AS NOVEL CYP2E1 INHIBITORS FOR THEIR PROTECTIVE EFFECT AGAINST XENOBIOTIC-INDUCED TOXICITY AND HIV REPLICATION ${ }^{3}$}

\section{Introduction}

Garlic, one of the world's most popular condiments, has a history of use for over 7000 years. Its culinary and medicinal properties are largely attributed to its complement of organosulfur compounds. Diallyl sulfide (DAS) is one of the major organosulfur compounds found in garlic, and it has been studied extensively as a preventive/protective agent against various pathological conditions such as cancer, HIV-1, diabetes, and Parkinson's disease [120]. DAS has attracted major attention for its inhibitory action on the cytochrome P450 2E1 (CYP2E1) enzyme and the resulting therapeutic/prophylactic benefits. CYP2E1 is a key metabolic enzyme for many xenobiotics such as alcohol and acetaminophen [39]. The inhibition of CYP2E1-mediated metabolism of these substrates, especially in overdoses, is crucially important in preventing cytotoxicity.

At low physiological concentrations, ethanol is metabolized by the enzymes alcohol dehydrogenase, catalase, and CYP2E1 [34]. However, at high concentrations, ethanol-induced CYP2E1 plays the predominant role in ethanol metabolism [39]. This results in increased production of reactive oxygen species (ROS) and other reactive metabolites, thereby causing hepatic and extra-hepatic toxicity [39, 236, 237, 286, 287]. Ethanol can also induce CYP2E1 in extra-hepatic cells such as monocytes and astrocytes, causing cytotoxicity to these cells due to increased metabolism and the resulting oxidative stress. The widely-used analgesic acetaminophen (APAP), another CYP2E1 substrate, is one of the safest medications in use today. However, it can cause severe hepatotoxicity at high doses though the CYP2E1-mediated metabolism of APAP and formation of a toxic metabolite [258]. APAP overdose has affected millions of people around the globe, especially in the United States, where it is the most common cause of acute liver failure [288]. In addition, CYP2E1 has also been found to be involved in HIV1 replication and HIV-1/viral protein-mediated toxicity [120]. A recent study by our own group has shown that CYP2E1 can be induced by viral proteins such as gp120 [141]. It also suggests that the HIV-1 viral proteins may cause increased production of ROS and reactive metabolites, consequently increasing the viral replication, possibly through the induction of CYP2E1.

DAS is known to be a selective CYP2E1 inhibitor [120] Many in vitro and in vivo studies have been conducted using DAS either to find the mechanistic pathways of CYP2E1-associated toxic effects or to examine its efficacy as an inhibitor of CYP2E1

\footnotetext{
${ }^{3}$ Reprinted from final submission with open access permission from Elsevier. Rahman M. A., Gong Y., Kumar S. In Vitro Evaluation of Structural Analogs of Diallyl Sulfide as Novel CYP2E1 Inhibitors for Their Protective Effect Against Xenobiotic-induced Toxicity and HIV Replication (2018). Toxicol Lett, 292, 31-38. doi: 10.1016/j.toxlet.2018.04.023
} 
[120]. However, DAS itself undergoes CYP2E1-mediated metabolism and produces toxic sulfur metabolites. Thus, there is a potential risk involved in using DAS as a therapeutic regimen. The challenge is to find a superior alternative to DAS with relatively low-tonegligible toxicity. We hypothesize that modification of the parent DAS structure would result in analogs with improved CYP2E1 inhibition capacity and reduced cytotoxicity. In our previous study, based on a computational ligand-docking analyses, we evaluated seven structural analogs (allyl methyl sulfide, allyl ethyl sulfide, diallyl ether, thiophene, 2-(prop-2-en-1-yloxy) ethan-1-amine, 5-hexen-1-amine, and 2-prop-2-enoxyacetamide) of DAS for their CYP2E1-inhibitory properties [289]. We found that two analogs (diallyl ether and allyl methyl sulfide) had greater CYP2E1 inhibitory capacity than DAS, while other analogs showed inhibitory capacity for CYP2E1 similar to DAS. In the current study, we used those seven analogs to evaluate their in vitro cytotoxicity and their ability to protect cells from ethanol- and APAP-mediated toxicity. We also tested these analogs for their efficacy in suppressing HIV-1 replication.

\section{Materials and Methods}

\section{Chemicals}

The compounds tested in this study were all purchased from commercial sources. Diallyl sulfide (purity 97\%), allyl methyl sulfide (AMS, purity 98\%), allyl ethyl sulfide (AES, purity 98\%), diallyl ether (DE, purity 98\%), thiophene (TP, purity 95-98\%), 2(prop-2-en-1-yloxy) ethan-1-amine (PEA, purity 95\%), and 5-hexen-1-amine (5, 1 HA, purity 95\%) were purchased from Sigma-Aldrich (St. Louis, MO). 2-prop-2enoxyacetamide (PEXA, purity 95\%) was purchased from Aldlab Chemicals (Woburn, MA). The stock solutions for these compounds were prepared in dimethyl sulfoxide (DMSO).

\section{Cell culture and treatment}

For this project, we used multiple relevant cell lines: human histiocytic lymphoma U937 monocytes, SVGA immortalized astrocytes, and HepaRG hepatocytes. These cells were used because CYP2E1 is constitutively expressed and is induced by ethanol leading to cytotoxicity in these cells $[39,116,290]$. We also used latently HIV-infected monocytic cells (U1 cells), which is considered the model system to study the effects of HIV in monocytes [244]. The U937 cell lines were obtained from ATCC (Manassas, VA). The U1 cell lines were obtained from the NIH AIDS Reagent Program (Germantown, MD). Both cell lines were cultured in Roswell Park Memorial Institute 1640 media (Sigma Aldrich, St. Louis, MO) supplemented with 10\% fetal bovine serum, L-glutamine, sodium bicarbonate, non-essential amino acids, and gentamycin (for U937 cells) and penicillin-streptomycin solution (for U1 cells). The SVGA astrocytes were a kind gift from Dr. Anil Kumar, University of Missouri-Kansas City (MO, USA), which were originally developed by Major et al. [291]. The SVGA cells were cultured in 
Dulbecco's Modified Eagle Medium supplemented with the same nutrients (except Lglutamine) as U937 media. The terminally-differentiated HepaRG cells were maintained in media prepared by the addition of the HepaRG Tox Medium Supplement ${ }^{\circledR}$ to $100 \mathrm{ml}$ of William's Medium E (ThermoFisher Scientific, Grand Island, NY) and $1 \mathrm{ml}$ of GlutaMAX. All the cells were maintained in a humidified incubator with $5 \% \mathrm{CO}_{2}$ at $37^{\circ} \mathrm{C}$. To compare the cytotoxicity, the cells were treated with DAS and its analogs every 12 hours for 2 days (acute treatment) or 3-7 days (chronic treatment), with the addition of fresh media during each treatment after the first one. DMSO $(\leq 0.5 \%)$ treatment served as the control condition for all experiments.

\section{Determination of CYP2E1 activity in U937 monocytic and SVGA astrocytic cells}

We demonstrated CYP2E1 enzymatic activity in U937 and SVGA cells. At first, we extracted membrane proteins and membrane-associated proteins from these cells using The Mem-PER Plus Kit following manufacturer's protocol. Briefly, the cells were permeabilized with a mild detergent to remove the soluble cytosolic proteins. A second detergent was then added to solubilize the membrane proteins. After extraction, we quantified the protein using the Pierce BCA protein assay kit. Finally, CYP2E1 activity was determined using Vivid ${ }^{\circ}$ CYP450 Screening Kit as described previously [245]. CYP2E1 baculosomes was used as positive control for this assay. All the above mentioned kits were purchased from ThermoFisher Scientific (Grand Island, NY).

\section{Measurement of reactive oxygen species (ROS) and cell viability using flow cytometry}

To determine the level of ROS and cell viability, U937 cells were treated with the analogs, and quantification was performed using flow cytometry (BD Biosciences, San Jose, CA). We used the fluorescence dye 5-(and-6)-chloromethyl 2', 7'dichlorodihydrofluorescein diacetate (CM- $\left.\mathrm{H}_{2} \mathrm{DCFDA}\right)$ (Life Technologies, Oregon, USA) for ROS measurement and Ghost dye (Tonbo Biosciences, San Diego, CA) for cell viability. After 48 hours of treatment, the cells were collected and washed with PBS. The cells were resuspended in PBS containing $2.5 \mu \mathrm{l}$ of CM- $\mathrm{H}_{2}$ DCFDA and $1 \mu \mathrm{l}$ of Ghost dye. They were incubated at $37^{\circ} \mathrm{C}$ without light for 30 minutes. After incubation, the cells were washed, resuspended in $300 \mu 1$ of PBS, and ROS and cell viability were measured. The data were analyzed using BD FACS software (version 8).

\section{XTT cell viability assay}

Cell viability was also measured using the XTT cell viability assay kit (Cell Signaling Technology Inc., Danvers, MA). In brief, $200 \mu$ of the media containing treated cells were collected and $50 \mu \mathrm{l}$ of XTT detection solution was added into each well of a $96-w e l l$ plate. The plate was incubated for $2-3$ hours in an incubator at $37^{\circ} \mathrm{C}$. The 
resulting absorbance, corresponding to relative viability, was measured at $450 \mathrm{~nm}$ using a Cytation 5 micro plate reader.

\section{LDH activity assay}

Cytotoxicity was also evaluated using the Pierce Lactate Dehydrogenase (LDH) Cytotoxicity Assay Kit (ThermoFisher Scientific, Grand Island, NY). LDH assay is a convenient method to measure cell viability in adherent cells. LDH is released into the media from damaged cells which indicates cytotoxicity and cytolysis. Briefly, $50 \mu 1$ of the treated media (in triplicates from each well) were collected into a 96-well plate and mixed with $50 \mu \mathrm{l}$ of the LDH reaction mixture. After 30 minutes of incubation at room temperature, the reaction was terminated by adding LDH stop solution. The absorbance was measured at $490 \mathrm{~nm}$ and $680 \mathrm{~nm}$ using the Cytation 5 micro plate reader.

\section{Caspase- 3 activity assay}

Activation of the caspase- 3 pathway is a hallmark of apoptosis. To measure caspase- 3 activity in the treated cells, we used a caspase- 3 colorimetric assay kit (BioVision, Inc., Milpitas, CA). After treatment, the cells were lysed using cell lysis buffer and protein was extracted. Then the protein concentration was determined using the BCA Protein Assay Kit (Pierce Biotechnology, Waltham, MA). About 50-100 $\mu \mathrm{g}$ of the protein was diluted to a final volume of $50 \mu 1$ in lysis buffer. Later, $50 \mu 1$ of $2 \mathrm{X}$ reaction buffer (containing $10 \mathrm{mM}$ DTT) and $5 \mu 1$ of $4 \mathrm{mM}$ DEVD-pNA substrate was added and incubated at $37^{\circ} \mathrm{C}$ for 1 hour in darkness. The absorbance was measured by using a Cytation 5 micro plate reader at a wavelength of $405 \mathrm{~nm}$.

\section{HIV p24 ELISA}

Suppression of the viral load was measured by using the p24 ELISA kit (ZeptoMetrix Corp, Buffalo, NY). The U1 cells were treated with the analogs and media was collected after 48 hours of treatment. The viral p24 group-specific antigen was specifically captured with a monoclonal antibody and then reacted with a high titered human anti-HIV-1 antibody conjugated with biotin. After incubating the sample with streptavidin-peroxidase, absorbance was measured using a micro plate reader. The OD value is proportional to the viral p24 load in the sample.

\section{Statistical analysis}

Data were analyzed using GraphPad Prism 5 software (GraphPad Software Inc., San Diego, CA). Data in this manuscript for all experiments are presented as mean \pm SEM of 3-6 experimental replicates. One-way ANOVA was used for comparisons 
between different treatment groups and $p$-values of $\leq 0.05$ were considered statistically significant.

\section{Results and Discussion}

We hypothesize that the modification of the parent DAS structure will not only improve the CYP2E1 inhibitory capacity of the analogs but will also reduce the potential toxicity at high concentrations. It is known that the sulfur hetero atom in the DAS structure interacts with the CYP2E1 active site and thereby acts as a competitive inhibitor [292]. However, this same phenomenon is also responsible for producing the toxic sulfur metabolites [120]. To find a superior alternative, we performed a structure-activity relationship study. We chose the commercially available DAS structural analogs in such a way that it would maintain its capacity to inhibit CYP2E1 while no longer being a substrate. The selected DAS analogs had either the oxygen as a hetero atom in place of sulfur (DE, PEA, and PEXA) or lacked the hetero atom completely (5,1 HA). These substitutions/deletion are likely to result in producing a non-toxic metabolite or no metabolite at all, mainly due to the absence of sulfur atom in the structure [120]. We also chose a DAS analog that had a stronger nucleophile at the carbon atom adjacent to the hetero atom (PEA, PEXA, and 5,1 HA). These alterations in DAS analogs are expected to result in improving their toxicity profiles. Furthermore, smaller side chains (AMS and AES) have been reported to increase the binding efficiency to the active site [120]. Unlike the other CYP enzymes, the binding site of the CYP2E1 lies in a narrow channel [293]. Hence, a low molecular weight cyclic compound (TP) is expected to work as a potent inhibitor but not as a substrate due to its cyclic structure. Our inhibition study showed that two analogs (DE and AMS) were stronger CYP2E1 inhibitors than DAS, TP had a similar inhibitory capacity to DAS, and the other analogs showed moderate CYP2E1 inhibition [294]. Despite the differences in the magnitude of their inhibition, we included all seven analogs for this in vitro toxicity and efficacy study. We used four different cells for this project: U937 monocytes, U1 monocytes, SVGA astrocytes, and HepaRG hepatocytes.

\section{CYP2E1 activity in U937 monocytic and SVGA astrocytic cells}

To demonstrate the efficacy of the DAS analogs as CYP2E1 inhibitor, we used three CYP2E1 expressing relevant cell lines in this study. Among them, HepaRG is a well-established terminally differentiated cell lines that mimics the characteristics (e.g. CYP expression) of the primary hepatocytes. In a separate study, we have shown that CYP2E1 is expressed in high level in HepaRG cells [48]. However, CYP2E1 is expressed in lesser magnitude in monocytic and astrocytic cells compared to hepatocytes. In our previous studies, we observed substantial CYP2E1 mRNA and protein level in U937 and SVGA cells [272, 295]. Since there is no report of CYP2E1 enzymatic activity in these cells, in this study, we examined CYP activity in these cells as described in Materials and Methods. We obtained around $100 \pm 10 \mu \mathrm{g}$ of protein from 5 million U937 cells and $180 \pm 10 \mu \mathrm{g}$ of protein from 7-8 million SVGA cells. We used various amount of 
protein to determine the concentration dependent increase in activity. The data showed that in both cell lines, CYP2E1 activity increased with time- and protein concentrationdependent manner (data not shown). CYP2E1 activity in U937 cells was comparatively higher than that in SVGA cells, which is consistent with our previous finding for CYP2E1 mRNA and protein levels in these cell lines [40]. CYP2E1 baculosomes showed linear increase in activity with time. This data justifies the use of these two cell lines for this current study, along with the HepaRG cells which resembles many characteristics of primary hepatocytes.

\section{Effect of acute treatment of DAS analogs on cytotoxicity}

At first, we conducted a time and concentration-dependent cytotoxicity study in U937 cells using the following commercially available analogs: DE, TP, AMS, and AES. We used this cell line to optimize time and concentration, because it is easily available and cost-effective compared to primary cells. We treated the U937 cells with 10-200 $\mu \mathrm{M}$ of DAS and its analogs for up to 48 hours. Using flow cytometry, we observed that DAS caused significant cytotoxicity at $50 \mu \mathrm{M}$, whereas DAS analogs did not show detectable toxicity (Figure 3-1A). At higher concentrations, e.g. $200 \mu \mathrm{M}$, DAS caused severe toxicity, whereas DE, TP, and AMS did not cause significant toxicity. We also measured the level of ROS. The results showed that DAS as well as its analogs did not cause increased ROS (Figure 3-1B). The results suggest that DAS and its analogs do not cause cytotoxicity through the oxidative stress pathway.

We performed further investigation using three other custom-made DAS analogs: PEA, PEXA, and 5,1 HA. Since $200 \mu$ M DAS caused maximum cytotoxicity, we used only this concentration with the custom-made DAS analogs (Figure 3-2). Using the XTT cell viability assay, we observed that after 48 hours of treatment, DE, TP, AMS, and PEXA did not cause any significant cytotoxicity in monocytes, astrocytes, or hepatocytes. The results further suggest that all the DAS analogs tested have better toxicity profiles than DAS, at least for acute treatment in both hepatic and non-hepatic monocytic and astrocytic cells.

\section{Effect on caspase-3 activity}

It has been reported that DAS is metabolized to reactive metabolites and cause toxicity through the caspase-3-dependent apoptotic pathway [296]. To determine whether the new DAS analogs show a lack of toxicity as a result of limited metabolism of these analogs into toxic species and subsequent lack of caspase- 3 cleavage activation, we treated the U937 cells with $200 \mu \mathrm{M}$ DAS analogs and measured caspase-3 activity. We observed that DAS, as expected, caused a $\sim 2$-fold increase in caspase-3 activity, whereas the DAS analogs did not cause any increase in activity (Figure 3-3). The results suggest that the DAS analogs do not induce caspase-3 apoptotic pathway. 


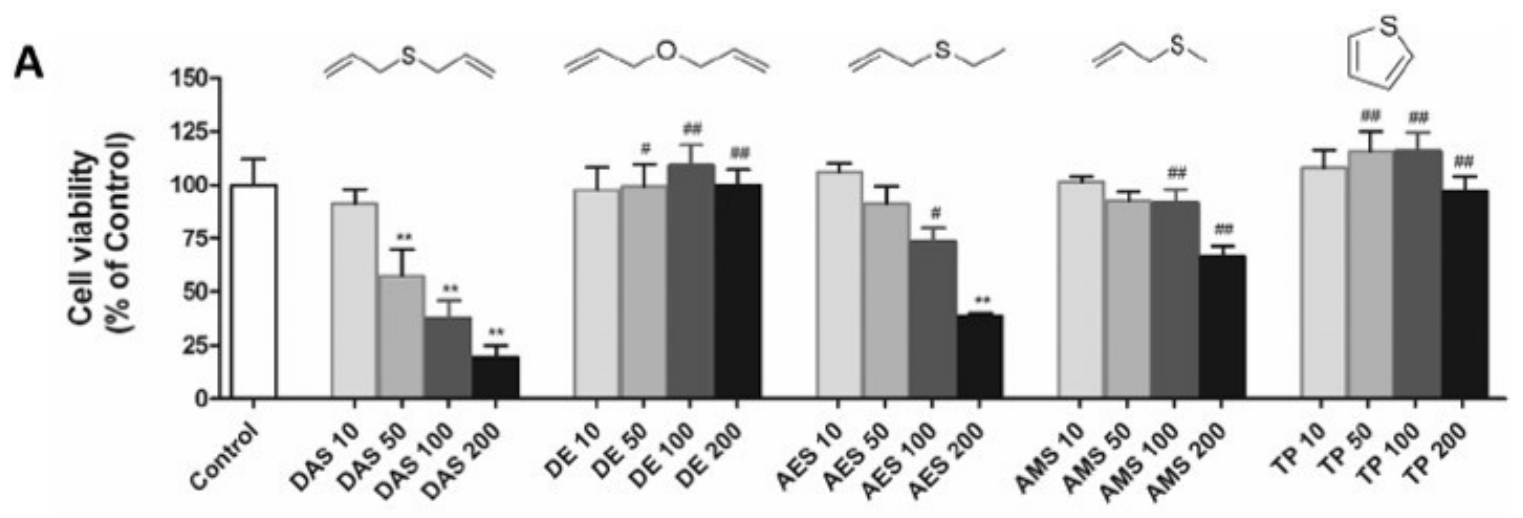

Analogs $(\mu \mathrm{M})$

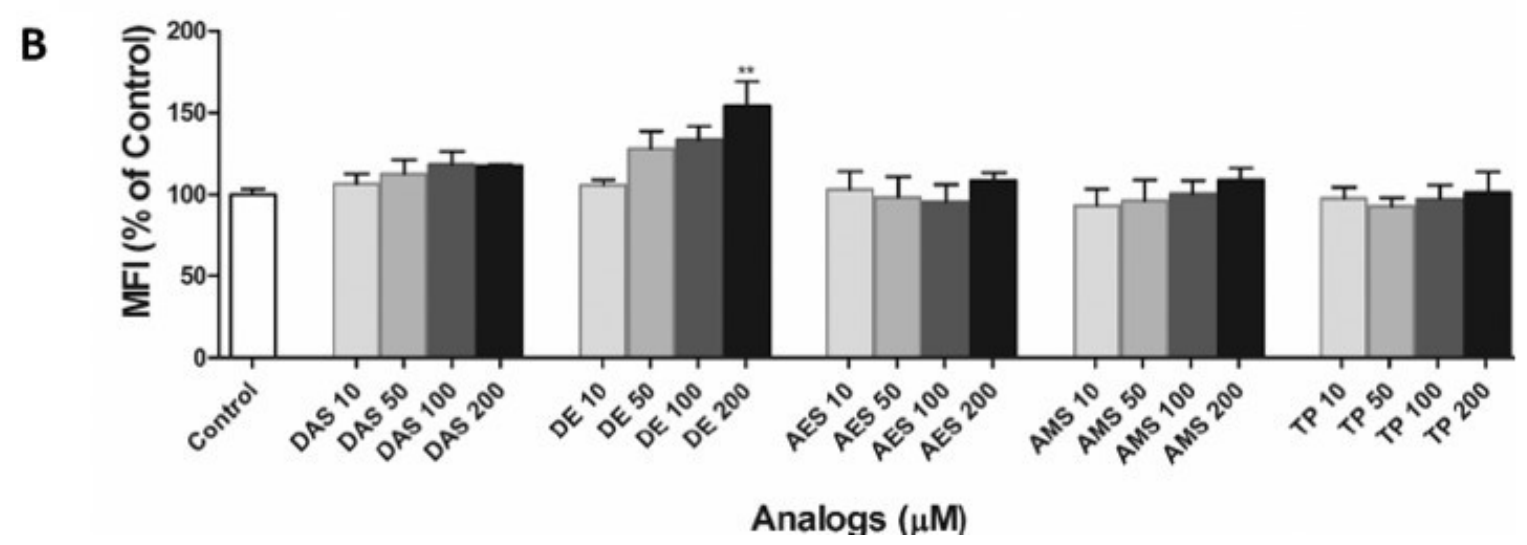

Figure 3-1. Effect of acute treatment on cell viability and ROS production in monocytes.

A) U937 cells were treated with DAS and the commercially available analogs DE, AES, AMS, and TP for 48 hours. Cell viability was quantified by flow cytometry. DAS caused severe toxicity at higher concentrations whereas DE, TP, and AMS did not show significant toxicity up to $200 \mu \mathrm{M}$. B) ROS production was quantified by flow cytometry. The mean fluorescent intensity (MFI) indicates the level of ROS. * indicates significance $(p<0.05)$ compared to control, \# indicates $(p<0.05)$ compared to DAS. 
A

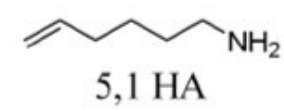

$\curvearrowright \mathrm{O} \mathrm{NH}_{2}$

PEA<smiles>C=CCOCC(N)=O</smiles>

PEXA

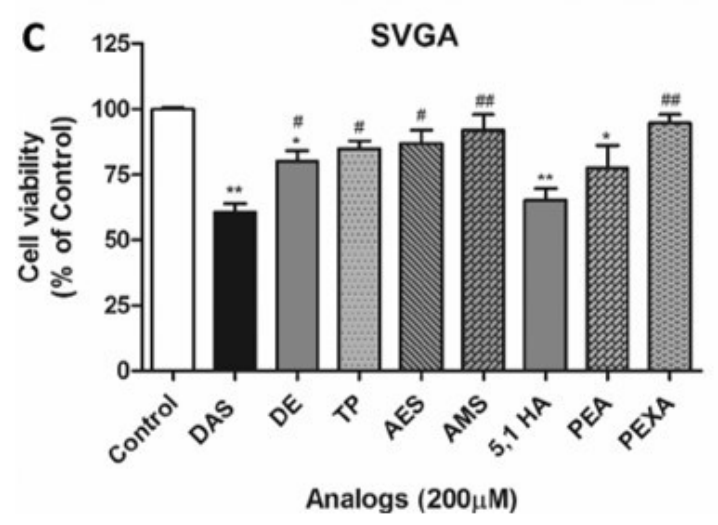

B

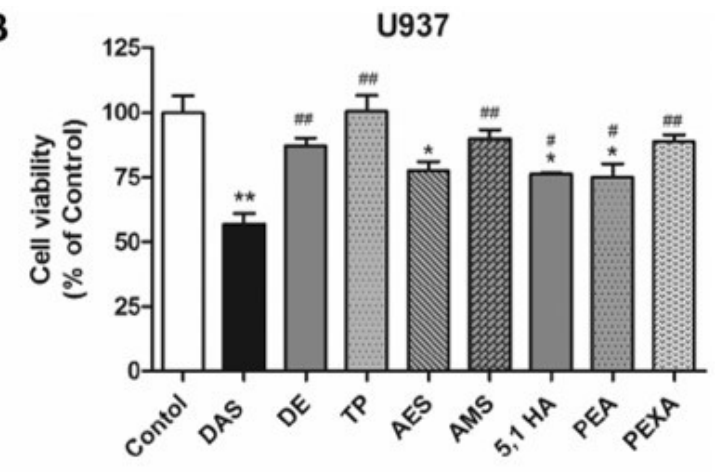

Analogs $(200 \mu \mathrm{M})$

D

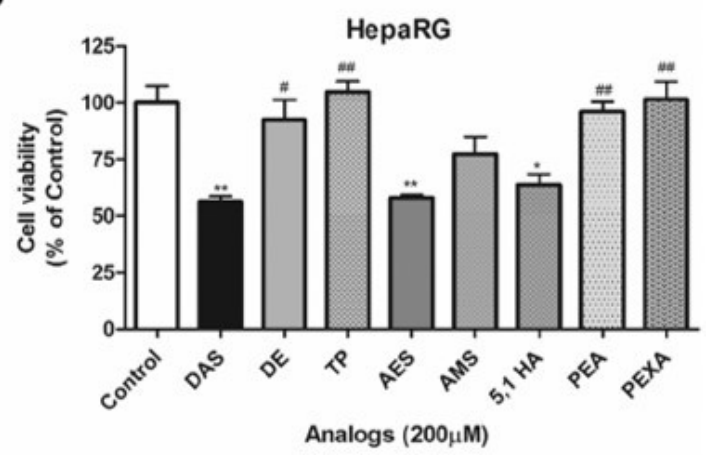

Figure 3-2. Effect of acute treatment on cell viability in monocytes, astrocytes, and hepatocytes.

A) The structure of the commercially available DAS analogs. B, C, and D) Cell viability in U937, SVGA, and HepaRG cells after 48 hour of treatment, as measured by XTT cell viability assay. * indicates significance $(\mathrm{p}<0.05)$ compared to control, \# indicates $(\mathrm{p}<0.05)$ compared to DAS. 


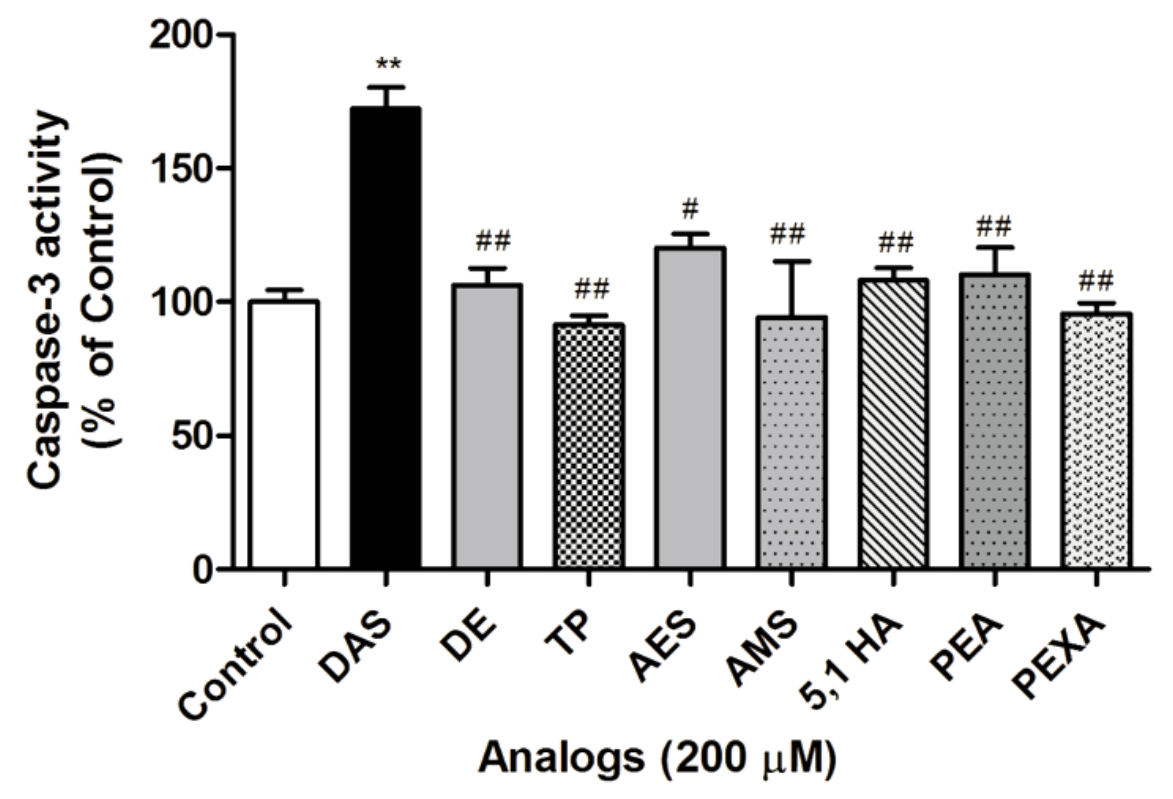

Figure 3-3. Effect on caspase-3 activity after treatment with DAS and its analogs. U937 cells were treated with $200 \mu \mathrm{M}$ of these compounds for 48 hours and caspase- 3 activity was measured by colorimetric assay. * indicates significance $(p<0.05)$ compared to control, \# indicates $(\mathrm{p}<0.05)$ compared to DAS. 


\section{Effect of chronic treatment on cytotoxicity with the selected DAS analogs}

One of the major concerns related to the use of DAS as a research tool or potential adjuvant therapy is that it has the potential to cause toxicity over an extended period, even at relatively low concentrations. Therefore, we performed a chronic treatment study using U937 cells. We treated the cells with varying concentrations $(5-200 \mu \mathrm{M})$ of the analogs for up to 7 days and visually examined cell viability every 24 hours under the microscope (Figure 3-4A). In addition, we performed XTT cell viability assays on day 4 and day 7 . The results showed that on the fourth day of treatment, all the analogs were significantly less cytotoxic than DAS at $200 \mu \mathrm{M}$ (Figure 3-4B). After seven days of treatment, the analogs showed some degree of toxicity at higher concentrations (100 and $200 \mu \mathrm{M}$ ), but still significantly less toxicity than DAS. We also treated the HepaRG hepatocytes at $200 \mu \mathrm{M}$ DAS and its analogs. We observed that DE, TP, 5,1 HA, PEA, and PEXA did not show any toxicity after 7 days of treatment. The results suggest that the new analogs have better toxicity profiles than DAS for even longer treatment regimens.

\section{Protective effect of the analogs from ethanol-induced toxicity}

Upon establishing the relative toxicity profiles of the novel DAS analogs using an in vitro system, we evaluated their efficacy in preventing xenobiotic-mediated toxicity and HIV-1 replication. Besides DAS being known as a selective CYP2E1 inhibitor, many studies have also shown that DAS administration induces anti-oxidant enzymes by modulating the Nuclear factor-erythroid 2 related factor 2 (Nrf2) pathway, which plays crucial role in maintaining redox homeostasis [124, 297]. Moreover, DAS has been shown to increase the reduced glutathione (GSH): oxidized glutathione (GSSG) ratio, which is indicative of increased antioxidant capacity [57]. We hypothesize that due to analogous chemical structure and CYP2E1 inhibitory property, the new DAS analogs will exert their protective function by inhibiting CYP2E1 mediated metabolism as well as by inducing antioxidant capacity. At first, we used these analogs to determine whether they can rescue ethanol-induced toxicity. Among all the alcohol-related disorders, alcoholic hepatitis is considered as the most severe form of alcohol-induced liver injury [298]. Unfortunately, there is currently no effective treatment regimen available on the market. Nearly half the patients suffering from alcoholic hepatitis do not benefit from the treatment [299]. Therefore, it is of critical importance to intervene the progression of ethanol-induced liver injury by taking preventive measures. CYP2E1-mediated ethanol metabolism and oxidative stress have been implicated as major contributors to alcoholrelated liver injury. CYP2E1 is also induced by ethanol, which further exacerbates tissue injury [39]. Ethanol can cause damage in the case of both regular/social and binge drinking scenarios.

We co-treated HepaRG hepatocytes with $50 \mathrm{mM}$ ethanol ( physiological concentration in binge and/or chronic drinking) and $50 \mu \mathrm{M}$ of the novel analogs for 48 hours. 

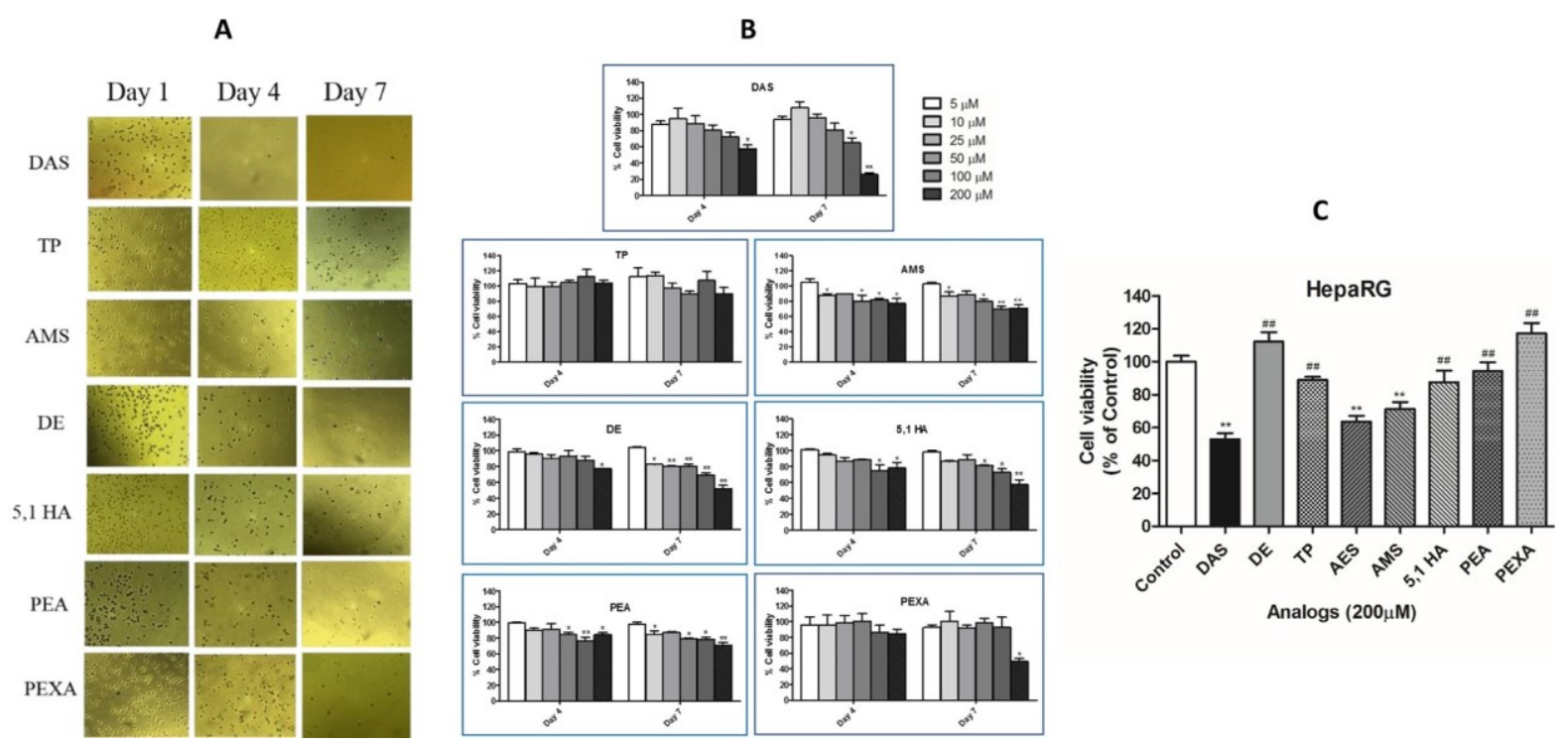

Figure 3-4. Effect of chronic treatment on cell viability in monocytes and hepatocytes.

U937 cells were treated with varying concentrations of DAS, TP, AMS, DE, and PEXA for 4-7 days. A) Microscopic images of the U937 cells of different treatment groups were taken on days 1, 4, and 7. B) Cell viability was quantified by XTT assay. * indicates significance $(\mathrm{p}<0.05)$ compared to $5 \mu \mathrm{M}$ of the respective analog. C) Hepatocytes were treated with $200 \mu \mathrm{M}$ of the compounds for seven days. Cell viability was quantified by XTT cell viability assay. ${ }^{*}$ indicates significance $(\mathrm{p}<0.05)$ compared to control, \# indicates $(p<0.05)$ compared to DAS. 
We optimized the concentration of DAS and its analogs based on our results from toxicity study, which showed that DAS was toxic at $50 \mu \mathrm{M}$ concentration, whereas the novel analogs did not cause any significant toxicity at $50 \mu \mathrm{M}$. Since the objective of this study was to find a suitable alternative to DAS which can be used at higher concentration for longer duration, we used this concentration to assess whether the new analogs can protect the hepatocytes from alcohol induced toxicity. The XTT cell viability assay showed that compared with DAS, DE, TP, AMS, PEA, and PEXA rescued ethanolinduced toxicity (Figure 3-5A). We also co-treated the cells with $20 \mathrm{mM}$ ethanol ( $\sim$ physiological concentration in regular/social drinking) and $20 \mu \mathrm{M}$ of the analogs for 48 hours, expecting that DAS would prevent ethanol-induced toxicity at a lower dose. We performed LDH cytotoxicity assay as a cytotoxicity marker for this experiment. Upon the damage of cell membrane, $\mathrm{LDH}$ is released into the cell culture media and indicates cytotoxicity. As expected, DAS rescued ethanol-mediated toxicity. However, TP, AMS, and PEXA showed even higher magnitudes of rescue for ethanol-induced toxicity than DAS (Figure 3-5B). Overall, these results are encouraging in the search for a better alternative to DAS in preventing ethanol-induced toxicity.

\section{Protective effect of DAS analogs from acetaminophen-induced toxicity}

Our next goal was to evaluate the efficacy of these DAS analogs in preventing APAP-induced toxicity. At elevated levels, CYP2E1-mediated metabolism of APAP produces the toxic metabolite- NAPQI, which potentiates liver injury. We co-treated the cells with $0.5 \mathrm{mM}$ APAP and $20 \mu \mathrm{M}$ of the analogs for 4 days. We measured the LDH activity in the media, which is indicative of enhanced cytotoxicity, every 24 hours for four days (Figure 3-6). Despite DAS being a CYP2E1 inhibitor, it did not rescue APAPmediated toxicity, but rather increased the toxicity further with APAP. However, TP, AMS, DE, PEA, and PEXA rescued APAP-induced toxicity. Furthermore, DAS showed a time-dependent increase in APAP-mediated toxicity, with severe toxicity after 4 days. However, TP, AMS, and DE showed protective effect against APAP even after 4 days of exposures. This further suggests that DAS is not an ideal CYP2E1 inhibitor to rescue APAP-mediated toxicity for chronic exposure. However, the DAS analogs do have the potential to inhibit CYP2E1 and rescue toxicity caused by chronic APAP exposure.

\section{Suppression of viral p24 antigen by DAS analogs}

HIV-1 viral proteins (e.g. gp120) have been implicated to increase HIV-related toxicity by inducing the CYP2E1 enzyme. Induction of CYP2E1 can result in increased oxidative stress and cytotoxicity via the metabolism of endogenous compounds, which in turn can increase viral replication [141]. We treated U1 (HIV-infected U937) monocytic cells with $20 \mu \mathrm{M}$ of the analogs. The results clearly demonstrated that the use of a CYP2E1 inhibitor can help reduce the viral load (p24) in the HIV-infected population. 
A

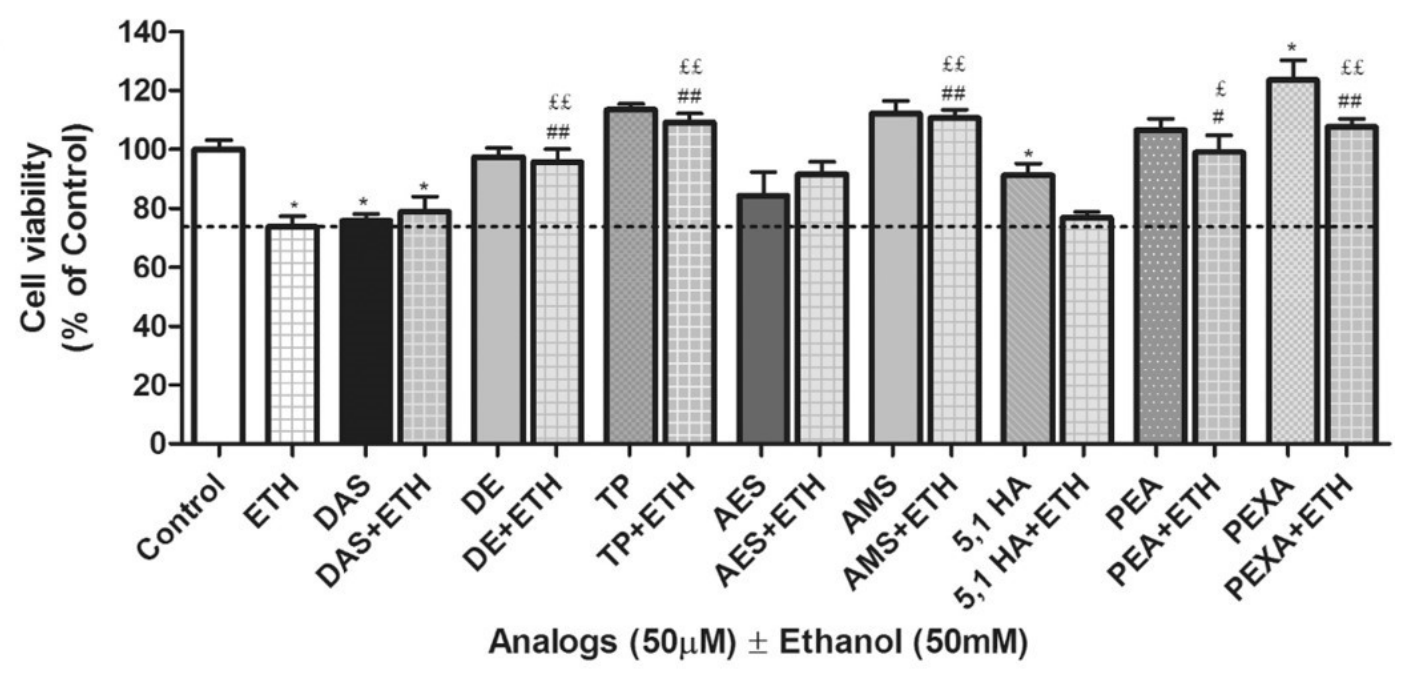

B

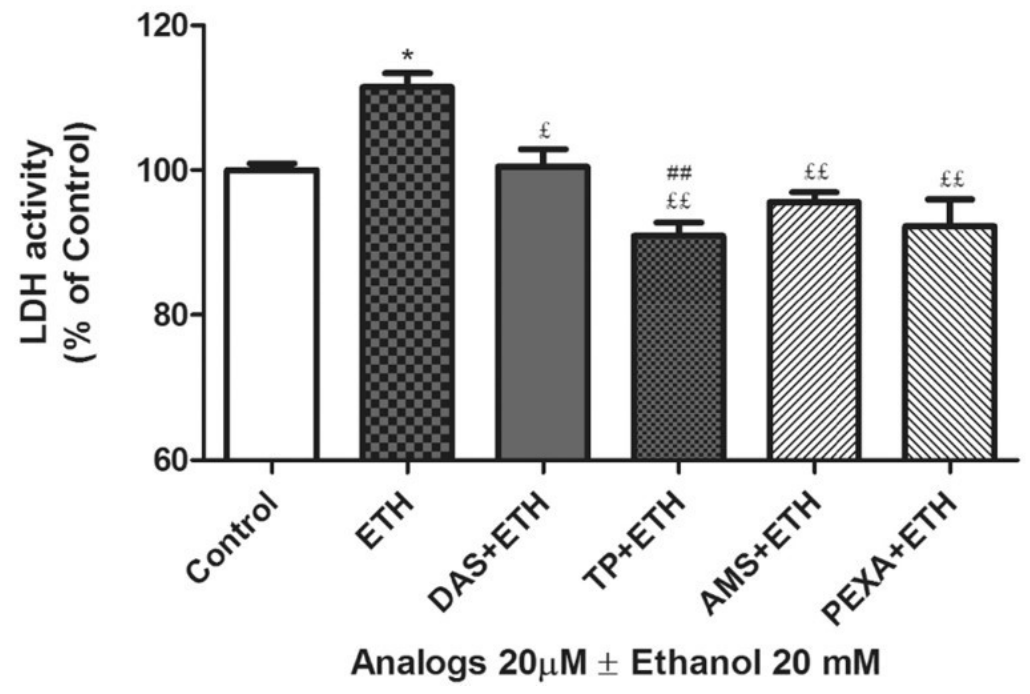

Figure 3-5. Rescue from ETH-induced toxicity in HepaRG hepatocytes.

A) Cells were co-treated with $50 \mathrm{mM}$ ethanol and $50 \mu \mathrm{M}$ of DAS and its analogs for 48 hours. XTT cell viability assay was performed to measure cell cytotoxicity. B) Cells were co-treated with $20 \mathrm{mM}$ ethanol and $20 \mu \mathrm{M}$ of the compounds for 48 hours. LDH activity assay was performed to evaluate comparative cytotoxicity. ${ }^{*}$ indicates significance $(\mathrm{p}<0.05)$ compared to control, £ indicates $(\mathrm{p}<0.05)$ compared to ethanol $(\mathrm{ETH})$, \# indicates $(p<0.05)$ compared to DAS+ETH. 
A

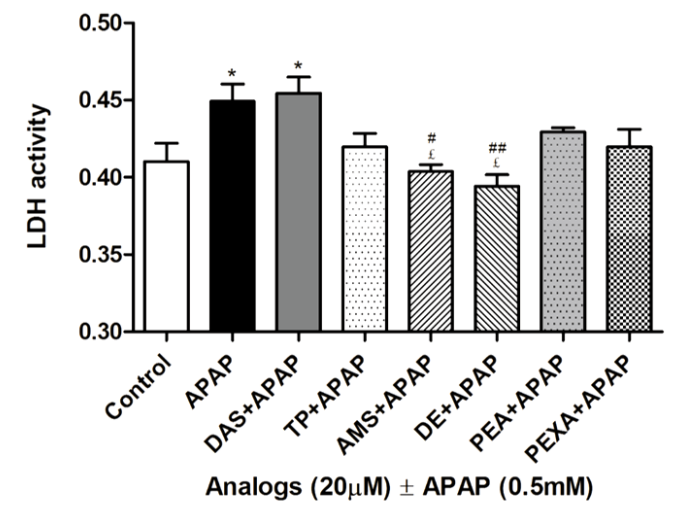

C

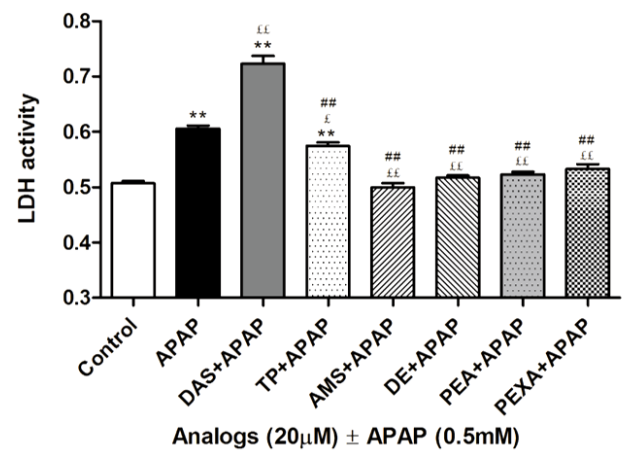

$\mathbf{B}$

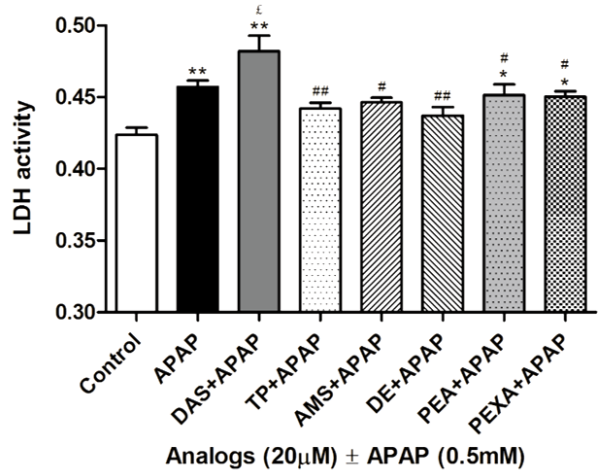

$\mathbf{D}$

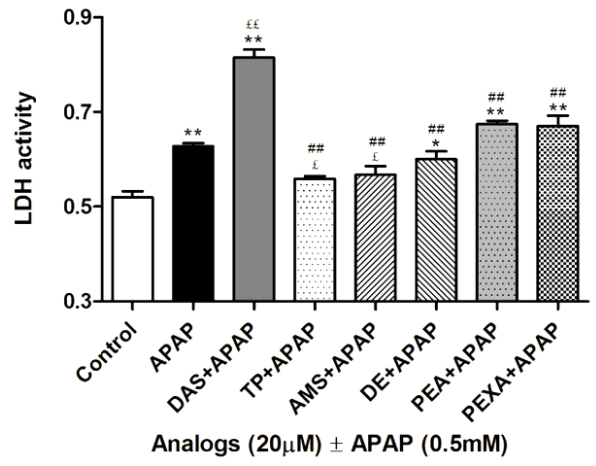

Figure 3-6. Rescue from APAP-induced toxicity in HepaRG hepatocytes.

A, B, C, and D) Cells were co-treated with $0.5 \mathrm{mM}$ ethanol and $20 \mu \mathrm{M}$ of DAS and its analogs for 48, 72, 96, and 120 hours. Cytotoxicity was measured by LDH activity assay. * indicates significance $(p<0.05)$ compared to control, $£$ indicates $(p<0.05)$ compared to APAP, \# indicates $(\mathrm{p}<0.05)$ compared to DAS+APAP. 
DAS, TP, AMS, and DE significantly lowered the viral load relative to the untreated control (Figure 3-7). In addition, TP and DE showed higher viral suppression than DAS, suggesting that these DAS analogs have better capability to suppress HIV-1 replication than DAS. This is an important finding that CYP2E1 inhibitors can reduce HIV replication.

\section{Conclusion}

Based on the results discussed above, we conclude that we have identified several analogs of DAS, especially TP, AMS, and DE. These compounds appear to be less toxic than DAS for the cellular models evaluated in this project. This is the first report of an extensive in vitro toxicity profile study of DAS and its analogs using hepatic and extrahepatic cells. Our next goal is to evaluate the pharmacokinetic and pharmacodynamic properties of these DAS analogs using an appropriate animal model. Upon further analysis, these analogs may replace DAS as a research tool and/or potential therapeutic regimen for preventing xenobiotic-induced toxicity and viral suppression. Despite having many therapeutic properties, the usage of DAS is restricted due to its toxicity. Therefore, there was a critical need for replacing DAS with a safer CYP2E1 inhibitor.

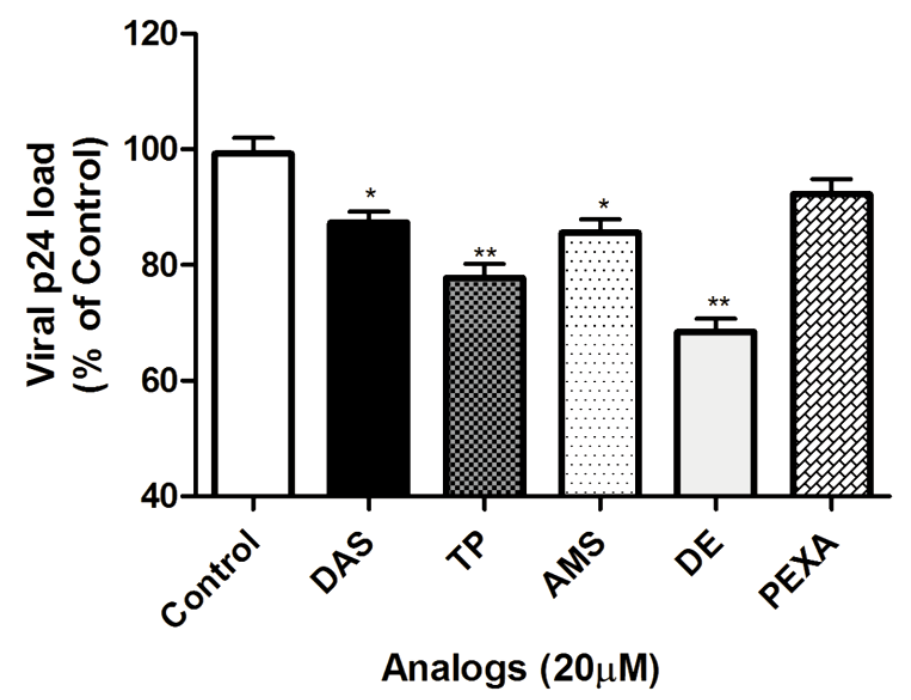

Figure 3-7. Suppression of viral load by DAS and its analogs in U1 cells.

Viral p24 load was quantified by p24 ELISA. * indicates significance $(\mathrm{p}<0.05)$ compared to control. 


\section{CHAPTER 4. PLASMA EXOSOMES EXACERBATE ALCOHOL- AND ACETAMINOPHEN-INDUCED TOXICITY VIA CYP2E1 PATHWAY ${ }^{4}$}

\section{Introduction}

Cytochrome P450 (CYP) enzymes are key components in drug metabolism and xenobiotic- induced toxicity. At high doses, oxidative stress and formation of toxic metabolites mediated by CYP2E1 are known as the principal pathway of toxicity induced by alcohol (ALC) and acetaminophen (APAP) [259, 300-302]. Ethanol-induced oxidative stress is not restricted to the hepatic cells, where ethanol is actively oxidized, but can affect various extra-hepatic tissues [34, 115, 287]. Since CYP2E1 is expressed in lower magnitude in the extra-hepatic regions than in the liver, the drug overdose-associated toxicity is not always clearly understood in extra-hepatic cells. Previously, we have shown that alcohol exposure increases human immunodeficiency virus (HIV) replication in monocytic cells via CYP2E1-mediated oxidative stress [237, 303]. However, the exact mechanism of alcohol exacerbated HIV replication in monocytic cells via CY2E1mediated oxidative stress is not clear.

Literatures suggest a potential contribution of exosomes as a key modulator in xenobiotics- and disease-induced toxicity in various tissue systems [304, 305]. Exosomes, the small extracellular nanovesicles produced by most cell types, are emerging as a novel tool for investigation of disease pathogenesis and drug discovery research [266, 306-309]. Exosomes can play a vital role in intra- and intercellular communication via selective packaging and transport of microRNAs (miRNAs), mRNAs, proteins, and other biological cargos to neighboring cells or to distant tissues via biofluids such as plasma, urine, cerebrospinal fluid, etc. [310-314]. Their unique ability to navigate through the biological system, transport important biomolecules, and influence the pathophysiology in the recipient cells make exosomes an attractive candidate for biological and biopharmaceutical research [315-317]. Recently, plasma exosomes have been studied extensively to discover reliable biomarkers for diseases such as cancer, kidney injury etc. [317-320]. Exosomes in plasma are secreted from a variety of tissues, and therefore, they are an excellent resource for examination of disease pathogenesis.

The presence of some of the most physiologically relevant CYP enzymes, including CYP2E1, in human plasma exosomes was recently reported by our group [321]. We observed that exosomes derived from plasma of healthy subjects packaged metabolically active CYP2E1 as well as other CYP isoforms. Interestingly, the level of CYP2E1 mRNA and protein level in plasma exosomes were significantly higher than the other CYPs such as CYP3A4. A recent report by our group demonstrated that there are significant differences in the packaging of inflammatory cytokines

\footnotetext{
${ }^{4}$ Reprinted from final submission with open access permission from Springer Nature. Rahman M. A., Kodidela S., Sinha N., Haque S., Shukla P.K, Rao R., Kumar S. Plasma Exosomes Exacerbate Alcoholand Acetaminophen-induced Toxicity via CYP2E1 Pathway (2019). Sci Rep, 9(1):6571. doi: 10.1038/s41598-019-43064-2.
} 
(e.g. interleukins- 6, 8, 10) in plasma exosomes isolated from HIV-infected ALC drinker [187]. Exposure of ALC or APAP to hepatic cells induces CYP2E1 and potentially increases packaging of CYP2E1 in exosomes [38, 115, 322]. Therefore, we hypothesized that these exosomes can transfer CYP2E1 to extra-hepatic tissues via biological fluids and mediate ALC- and APAP-induced toxicity.

In this study, we examined whether plasma exosomal CYP2E1 cargo play a role in exacerbating ALC- and APAP-induced toxicity in hepatic and extra-hepatic cells.

\section{Materials and Methods}

\section{Chemicals}

Acetaminophen (catalog \#A5000-100G) and diallyl ether (purity 98\%, catalog \# 259470) were purchased from Sigma-Aldrich (St. Louis, MO). Two hundred proof ethanol (catalog \#A405P4) was purchased from Fisher Scientific (Hampton, NH).

\section{Cell culture and treatment}

For the current study, two types of cell lines were used: 1) HepaRG hepatocytes and 2) latently HIV-1-infected monocytic U1 cells. Terminally differentiated HepaRG cells (ThermoFisher Scientific, Grand Island, NY) are the most reliable and appropriate cell lines for hepatocyte research due to their similar expression profile of CYPs and other metabolic enzymes when compared with primary hepatocytes [323]. The U1 cells (NIH AIDS Reagent Program; Germantown, MD) are recognized as the model system for investigating HIV effects in monocytes [324]. HepaRG cells were cultured in media consisting of William's E Medium supplemented with HepaRG Tox/Maintenance Medium Supplement ${ }^{\circledR}$ and Glutamax. First, the cells were plated using maintenance media. After 24 hours, maintenance media was replaced with tox media and cells were allowed to reconstitute in monolayer. The treatment was started at the $7^{\text {th }}$ day since the peak CYP expression occurs from day 7-10. The U1 cells were cultured in Roswell Park Memorial Institute (RPMI) 1640 media (Sigma Aldrich, St. Louis, MO) supplemented with 10\% fetal bovine serum, L-glutamine, sodium bicarbonate, non-essential amino acids, and penicillin-streptomycin solution. The cells were maintained in a humidified incubator with $5 \% \mathrm{CO}_{2}$ at $37^{\circ} \mathrm{C}$. Cells were co-treated with either $50 \mathrm{mM}$ ethanol or 0.5 $\mathrm{mM}$ acetaminophen along with isolated human or mouse plasma exosomes for 1 - 8 days. We used the CYP2E1 inhibitor diallyl ether $(20 \mu \mathrm{M})$ to validate the role of plasma exosomal CYP2E1 in ALC- or APAP-induced toxicity. Cell culture media was collected for use in the LDH cell viability assay and replaced with an equivalent amount of fresh media. At the end of treatment, cells were harvested for performing protein extraction and western blot assay. 


\section{Isolation and validation of exosomes from human and mouse plasma}

Exosomes were isolated from both human and mouse plasma for this project. Human blood samples were collected from de-identified healthy individuals at Interstate Blood Inc. (Memphis, TN). Exosomes were isolated using the Plasma Exo Kit (Applied Biosystems, Foster City, CA). In brief, a $0.22 \mu \mathrm{m}$ filter was used to remove larger vesicles $(>200 \mathrm{~nm})$ from the plasma. Afterwards, the human and mouse samples were centrifuged at 2,000 and 10,000 g, respectively for $20 \mathrm{~min}$ to remove cell debris. To the clarified plasma, 0.5 volume of $1 \mathrm{X}$ PBS was added and mixed thoroughly. Afterwards, 0.2 volume of exosome precipitation reagent was added, and the samples were mixed well by vortexing. After a $10 \mathrm{~min}$ incubation at room temperature, samples were centrifuged at 10,000 $\mathrm{g}$ for $5 \mathrm{~min}$. The resulting pellet was resuspended using appropriate medium and used for downstream procedures. We checked for expression of the standard exosomal marker proteins such as CD63 and CD81 by western blotting. We also tested whether plasma exosomes contained a liver-specific HP protein, since the liver is one of the major contributors of plasma exosomal CYP enzymes. Exosomes were also validated in terms of size, shape, and quality by using Transmission Electron Microscopy, following a standard protocol as described previously [325] (JEOL 2000EXII TEM, Neuroscience Institute, The University of Tennessee Health Science Center).

\section{Acetylcholine esterase assay}

The quality of the isolated exosomes was further verified by measuring acetylcholinesterase activity using the validated Amplex ${ }^{\mathrm{TM}}$ Red Acetylcholine/Acetylcholinesterase Assay Kit (Molecular Probes, Invitrogen). In brief, exosome pellets were resuspended in $100 \mu \mathrm{l}$ of $50 \mathrm{mM}$ Tris- $\mathrm{HCl}(\mathrm{pH} \mathrm{8.0)}$ ) and incubated with $100 \mu \mathrm{L}$ of the Amplex Red reagent containing $2 \mathrm{U} / \mathrm{mL}$ HRP, $0.2 \mathrm{U} / \mathrm{mL}$ choline oxidase, and $100 \mu \mathrm{M}$ acetylcholine in a final volume of $200 \mu \mathrm{L}$. Acetylcholinesterase and $\mathrm{H}_{2} \mathrm{O}_{2}$ solution were used as a positive control. The reaction plate was incubated in the absence of light for an hour at room temperature. Fluorescence measurement was taken every $15 \mathrm{~min}$ at a wavelength of $530 \mathrm{~nm}(\lambda \mathrm{ex})$ and $590 \mathrm{~nm}(\lambda \mathrm{em})$, respectively.

\section{Animal treatment}

In the present study, we used 10- to 12 -week old C57BL/6 female mice. The animal study protocol was approved by the Institutional Animal Care and Use Committee (IACUC, approval number 18-086) of the University of Tennessee Health Sciences Center (Memphis, TN, USA). Mice were divided into two groups, a control $(n=6)$ and a treatment group $(n=6)$. The control mice received Lieber-DeCarli control liquid diet and the treatment group mice received a single dose of ethanol $\left(5 \mathrm{~g} \mathrm{~kg}^{-1}\right)$. The animals were sacrificed at the end of the study and blood was collected to isolate plasma. Further, we isolated exosomes from plasma using a validated exosome isolation kit (Invitrogen, Life Technologies, NY) and characterized as described in the preliminary study and previous reports [321]. 


\section{Exosomes labeling and uptake}

After treating cells with isolated plasma exosomes, the cellular uptake of the exosomes was measured by using Exo-GLOW ${ }^{\mathrm{TM}}$ Exosome Labeling Kits (System Biosciences, CA). Exosome pellets containing 100-500 $\mu \mathrm{g}$ protein were resuspended in $500 \mu 1$ of $1 \mathrm{X}$ PBS. Next, $50 \mu \mathrm{l}$ of 10X Exo-Green fluorescent label was added to the solution, mixed well by inversion, and incubated at $37^{\circ} \mathrm{C}$ for $10 \mathrm{~min}$. To this mixture, $100 \mu \mathrm{l}$ of ExoQuick-TC reagent was added (mixed by inversion) to stop the labeling reaction and kept on ice (or at $4{ }^{\circ} \mathrm{C}$ ) for 30 minutes. The samples were centrifuged at $16000 \mathrm{~g}$ for 3 minutes and the washed pellet was resuspended in $500 \mu 1$ of $1 \mathrm{X}$ PBS. The labeled exosome suspensions were exposed to hepatocytes and their uptake was monitored by fluorescence microscopy.

\section{SiRNA transfection and treatment}

We blocked CYP2E1 expression in HepaRG cells by transfecting the cells with predesigned human CYP2E1 siRNA or scrambled siRNA, followed by exosome treatment. Following 72-hour incubation with lipofectamine transfection reagent in serum-free and antibiotic-free media, siRNA-transfected cells were treated with plasma exosomes \pm ALC and toxicity was compared with non-transfected treatment group to measure the specific contribution of plasma exosomal CYP2E1 in enhancing ALCinduced toxicity. Toxicity was measured by LDH assay.

\section{Western blot}

Protein expression in the exosomes derived from human and murine plasma was determined by western blotting. Protein quantification was carried out by using a Pierce $^{\mathrm{TM}}$ BCA protein assay (ThermoFisher Scientific, Grand Island, NY) as described before. Approximately $10 \mu \mathrm{g}$ of protein was loaded into a polyacrylamide gel. The gel was run for $90 \mathrm{~min}$ at $150 \mathrm{~V}$, which separated the proteins based on their molecular weight. To transfer the proteins from the gel to a polyvinyl fluoride membrane, gel was run for 90 minutes at $0.35 \mathrm{Amp}$. After the proteins were transferred to the membrane, it was blocked with 5-10 ml of Li-Cor blocking buffer (LI-COR Biosciences, Lincoln, NE) for 60 minutes to prevent the nonspecific binding of antibodies to its surface. The membrane was then incubated overnight with primary antibodies (CD63 rabbit Mab, 1:400 dilution, Proteintech catalog \#25682-1-AP; CD81 rabbit Mab 1:400 dilution, Santa Cruz Biotechnology, catalog \#sc-9158; HP rabbit Mab, 1:500 dilution, Proteintech, catalog \#16665-1-AP ; CYP2E1 rabbit Mab, 1:400 dilution, Millipore, catalog \#AB1252; SOD1 mouse Mab, 1:200 dilution, Santa Cruz Biotechnology, catalog \#sc-101523; Catalase rabbit Mab, 1:400 dilution, Proteintech, catalog \#21260-1-AP) at $4{ }^{\circ} \mathrm{C}$. The membrane containing blots was washed with PBS containing $0.2 \%$ Tween-20 several times before incubating with the corresponding secondary antibodies for $1 \mathrm{~h}$ at room 
temperature, protected from light. The Image Studio Lite version 4.0 in a Li-Cor Scanner (LI-COR Biosciences) was used to scan the membrane blots. The fold change in the expression of proteins was calculated based on densitometry data obtained from the Image Studio Lite software.

\section{LDH cell viability assay}

Cytotoxicity was measured using the Pierce Lactate Dehydrogenase (LDH) Cytotoxicity Assay Kit (ThermoFisher Scientific, Grand Island, NY) following the manufacturer's protocol. Release of LDH into the cell culture media is an indicator of cytotoxicity and cytolysis. In brief, $50 \mu \mathrm{l}$ of the collected media were mixed with $50 \mu \mathrm{l}$ of the LDH reaction mixture in a 96-well plate. After incubating at room temperature for 30 minutes, the reaction was stopped by adding LDH stop solution. Using a micro plate reader (Cytation ${ }^{\mathrm{TM}} 5$ Cell Imaging Multi-Mode Reader, BioTek, VT), the absorbance was measured at $490 \mathrm{~nm}$ and $680 \mathrm{~nm}$. Higher absorbance indicated higher toxicity.

\section{Statistical analysis}

Data analysis was carried out using GraphPad Prism 5 software (GraphPad Software Inc., San Diego, CA). All the data are presented as mean \pm SEM of 3-6 experimental replicates. One-way ANOVA followed by Tukey's post hoc test was used for comparisons between different treatment groups and $p$-values of $\leq 0.05$ were considered statistically significant.

\section{Results}

\section{Identification and characterization of plasma exosomes and uptake of exosomes in the recipient cells}

We isolated exosomes from healthy human plasma as previously described and characterized their physicochemical properties[48, 325]. Transmission electron microscopy (TEM) images confirmed the presence of exosomes (double-membraned vesicles) in the prepared sample (Figure 4-1A). Further, we showed a time-dependent increase in acetylcholine esterase activity (Figure 4-1B) and the presence of CD63 and CD81 (Figure 4-1C), which are specific markers for exosomes, confirming the identification of exosomes. Further, we showed the presence of haptoglobin (HP) (Figure 4-1C), a specific marker protein for hepatocyte-derived exosomes, suggesting the presence of liver-derived exosomes in the plasma. Since liver is the powerhouse of CYP enzymes, it is likely that the majority of the CYP enzymes in the plasma exosomes are secreted from the liver. Moreover, CYP enzymes in plasma exosomes may come from a variety of other cells as well since CYPs are also expressed in many extra-hepatic tissues such as lung, kidney, etc. Previously, we have shown that plasma exosomes 
a

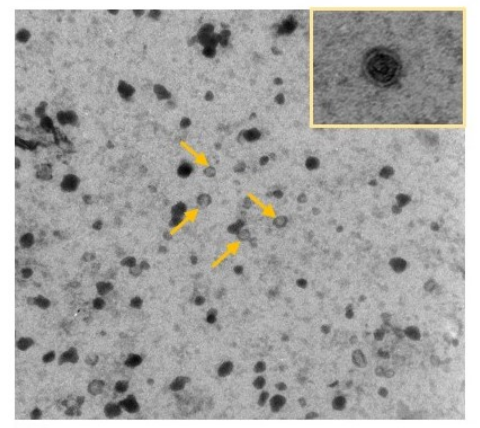

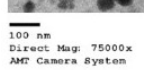

d
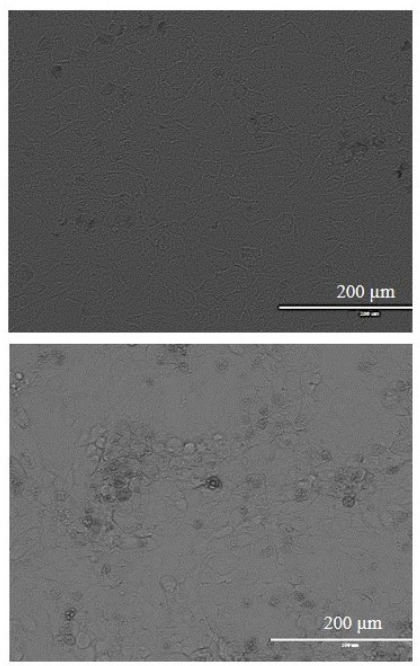

Bright Field b

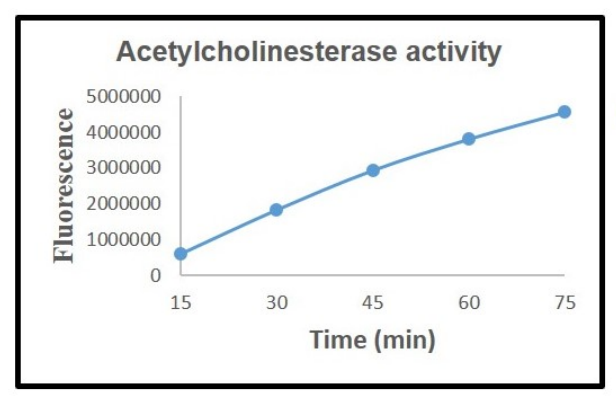

c
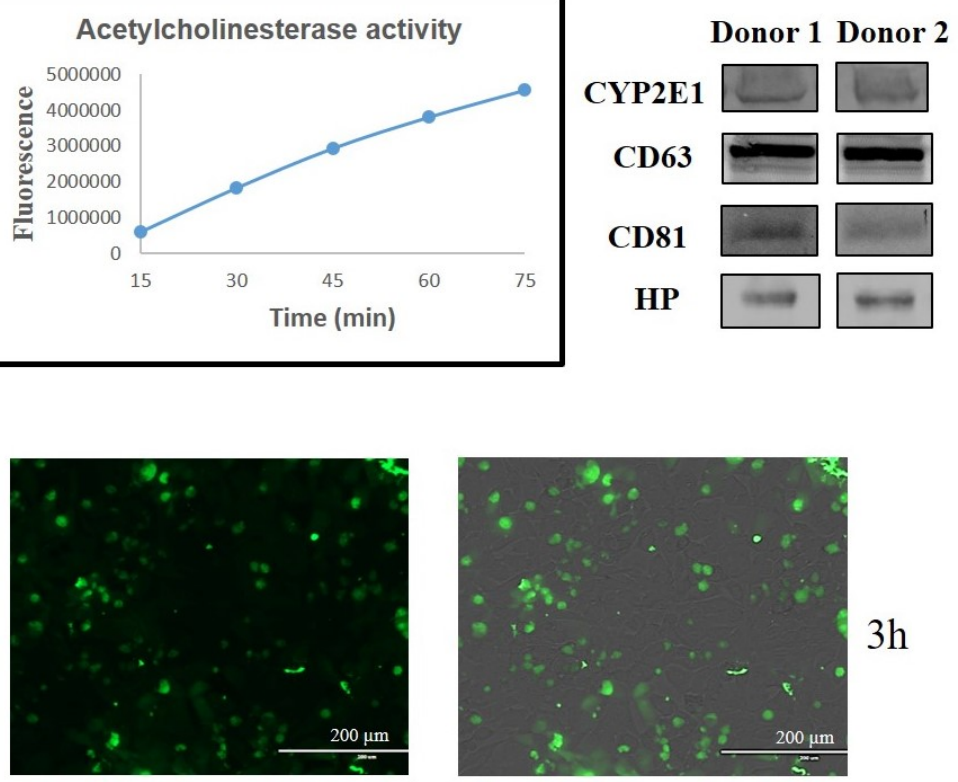

$3 \mathrm{~h}$

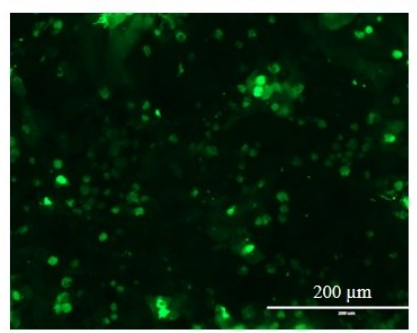

GFP

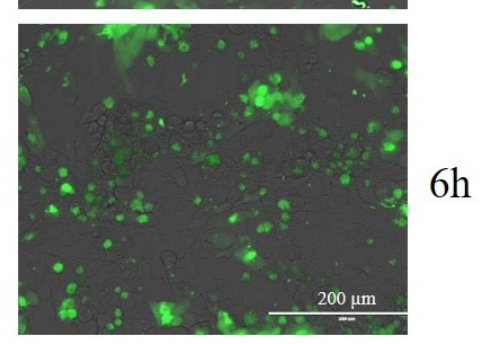

Overlay

Figure 4-1. Identification of exosomes derived from human plasma.

A) Transmission electron microscope (TEM) images of exosomes (arrows indicate exosomes). B) Acetylcholinesterase activity in exosomes. C) Representative immunoblots for the expression of exosomal marker protein CD63 and CD81, and liver specific exosomal protein HP in plasma exosomes. Equal amount of protein $(10 \mu \mathrm{g})$ was loaded in each lane. D) Bright field, fluorescent, and overlay images of cells after 3 and 6 hours of exosomes exposure. Microscope was set to visualize particles of $\leq 200 \mu \mathrm{m}$ diameter. Bright field and fluorescent images represent only cells and cells with exosomes, respectively. Overlay images demonstrate the presence of exosomes within the cells. 
contain substantial amount of CYP2E1 mRNA and protein in healthy human donor[48]. In this study, we also found that plasma exosomes from two healthy donors contained significant level of CYP2E1 protein (Figure 4-1C).

To determine whether the exosomes are involved in cell-cell communication, we performed an exosome uptake assay. In this study, we used HepaRG hepatocytes and the HIV-1-infected monocytic U1 cell line. HepaRG cells are considered a reliable in vitro model for studying hepatic drug metabolism and liver-related pathologies. They express the majority of phase I and II metabolic enzymes, including CYP2E1, and CYP2E1 is further induced by ALC in HepaRG cells [326-328]. Similarly, among extra-hepatic cells, HIV-infected monocytic cells (U1) express cellular CYP enzymes, especially CYP2E1 and are induced by ALC exposures [115, 237]. We exposed plasma exosomes to HepaRG cells. As shown previously [325] with U1 cells, our fluorescence microscopy imaging showed that exosomes are readily taken up by the recipient hepatocytes after 3-6 hours of exposure (Figure 4-1D).

\section{Effect of human plasma exosomes on hepatocytes upon ALC and APAP exposure}

In our previous study, we observed that plasma exosomes contain large amounts of CYP2E1 enzyme relative to hepatocytes or hepatocyte-derived exosomes [48]. To investigate the effect of plasma exosomal CYP2E1, we co-treated HepaRG cells with exosomes (derived from $50 \mu \mathrm{L}$ of clarified plasma) along with $50 \mathrm{mM}$ ALC and $0.5 \mathrm{mM}$ APAP. As expected, both ALC and APAP showed time-dependent increase in toxicity in HepaRG cells, which are known to be mediated through CYP2E1 pathway. We further observed that treatment with plasma exosomes caused a significant increase in ALCinduced toxicity in a time-dependent manner (Figure 4-2A). Similarly, plasma exosome exposure resulted in increased APAP-induced toxicity (Figure 4-2B). These results suggested that CYP2E1 cargo in plasma exosomes may contribute to the toxicity induced by ALC and APAP. It is important to note that plasma exosomes treatment alone caused significant toxicity from $5^{\text {th }}$ day onwards, perhaps due to increased metabolism of endogenous substrates as well as delivery of oxidative stress related miRNA (e.g. mir200 family) resulting in enhanced oxidative stress and cytotoxicity.

\section{The role of plasma exosomal CYP2E1 in mediating ALC- and APAP-induced toxicity}

To examine whether plasma exosomal CYP2E1 contributes to ALC- and APAPinduced toxicity, we pretreated the hepatic cells with selective CYP2E1 inhibitor and siRNA. In our laboratory, we have identified several analogs of diallyl sulfide (DAS) as more potent and relatively safer CYP2E1 inhibitors. Diallyl ether (DE) is one of the analogs with the best CYP2E1 inhibitory capacity and lowest toxicity profile [289, 329]. We pretreated HepaRG cells with ALC and/or APAP and plasma exosomes with or without pre-treatment with DE. In both set of treatments, we observed that DE 
a
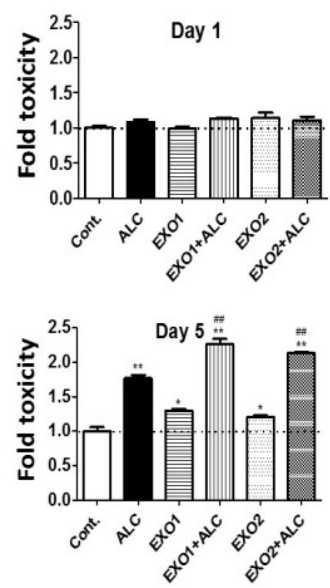
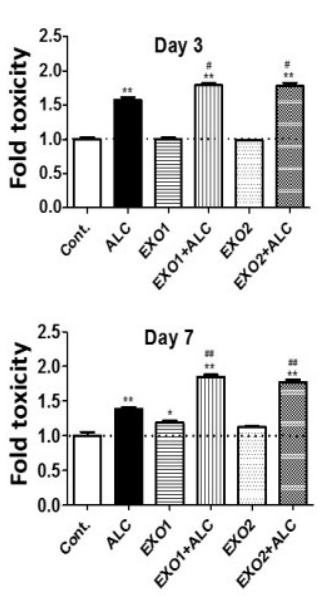

b
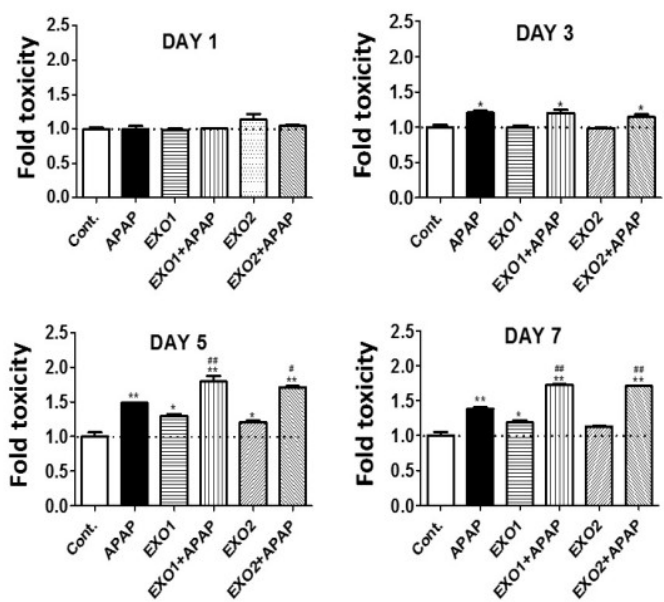

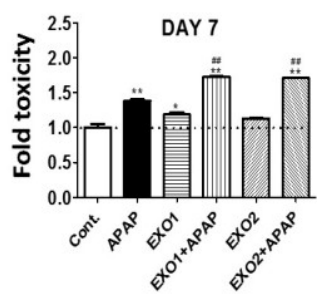

Figure 4-2. Effect of plasma exosomal CYP2E1 in HepaRG cells upon ALC and APAP treatment.

Plasma exosomes were isolated from healthy human plasma and then treated to HepaRG cells \pm ALC and APAP (panel A \& B respectively). EXO1 and EXO2 represent exosomes isolated from two different volunteers. Cytotoxicity was measured every day using LDH cell viability assay. The data shown here represent $n \# 3$ experiments. One-way ANOVA was used to measure statistical significance. * represents significance when compared to control, \# represents significance when compared to ALC/APAP. 
significantly reduced the additional toxicity caused by co-treatment of ALC/APAP and plasma exosomes (Figure 4-3A\&B). To further confirm the potential impact of plasma exosomal CYP2E1 in ALC-induced toxicity, we silenced the cellular expression of CYP2E1 in HepaRG hepatocytes with CYP2E1 siRNA and then exposed cells with ALC + exosomes. As expected, we observed that CYP2E1 siRNA treatment significantly rescued the cells from ALC-induced toxicity, while it did not reduce/abolish exosomeinduced toxicity (Figure 4-3C).

\section{Effect of human plasma exosomes on $\mathrm{U} 1$ monocytic cells}

We previously reported that ALC-induced CYP2E1 expression in monocytic/macrophage cell lines resulted in increased oxidative stress and cytotoxicity [118]. It is likely that addition of external CYP2E1 load into an in vitro system will exacerbate CYP2E1-mediated toxicity in U1 cells. Therefore, we treated U1 cells with 50 $\mathrm{mM} \mathrm{ALC} \mathrm{(physiological} \mathrm{concentration} \mathrm{of} \mathrm{ethanol} \mathrm{in} \mathrm{binge} \mathrm{drinking} \mathrm{[330])} \mathrm{and} 0.5 \mathrm{mM}$ APAP (sub-toxic physiological concentration [331]) along with plasma exosomes. After 2 days, we observed that plasma exosome treatment caused increased toxicity with both ALC and APAP co-treatment (Figure 4-4 A\&B). This result further suggests that plasma exosomal CYP2E1 cargo can have significant physiological effects not only in hepatic, but in extra-hepatic cells as well.

\section{Effect of alcohol on exosomal CYP2E1 derived from mice plasma}

To verify our in vitro findings, we isolated exosomes from the plasma of mice treated with and without alcohol (binge exposure). Although the role of CYP2E1 in alcohol-induced liver toxicity is well known in alcohol-drinking mice model, the contribution of plasma exosomes via CYP2E1 in toxicity is unknown. Therefore, we measured changes in the protein expression of CYP2E1 and two antioxidant enzymes (AOEs), SOD1 and catalase along with exosomal marker protein CD63 (Figure 4-5A). Interestingly, the average level of CD63 was reduced in alcohol-treated mice. The most crucial observation, however, was an increase in the level of CYP2E1 in binge-drinking mice (Figure 4-5A). The levels of the AOEs, SOD1 and catalase, were also decreased, suggesting an overall increase in oxidative stress components in the exosomes derived from ALC-treated mice.

\section{Effect of mouse plasma exosomes on $\mathrm{U} 1$ cells upon ethanol treatment}

Since we observed increased CYP2E1 and reduced AOE expression in exosomes derived from ALC-treated mice, we anticipated that this phenomenon will contribute to enhanced ALC-induced toxicity in the recipient cells. To examine this, we co-treated U1 cells with $50 \mathrm{mM} \mathrm{ALC} \pm$ exosomes isolated from control or binge-drinking mice. After 4 days of ALC treatment, we observed that exosomes derived from ALC-exposed mice caused significantly higher toxicity than exosomes from the control group (Figure 4-5B). 

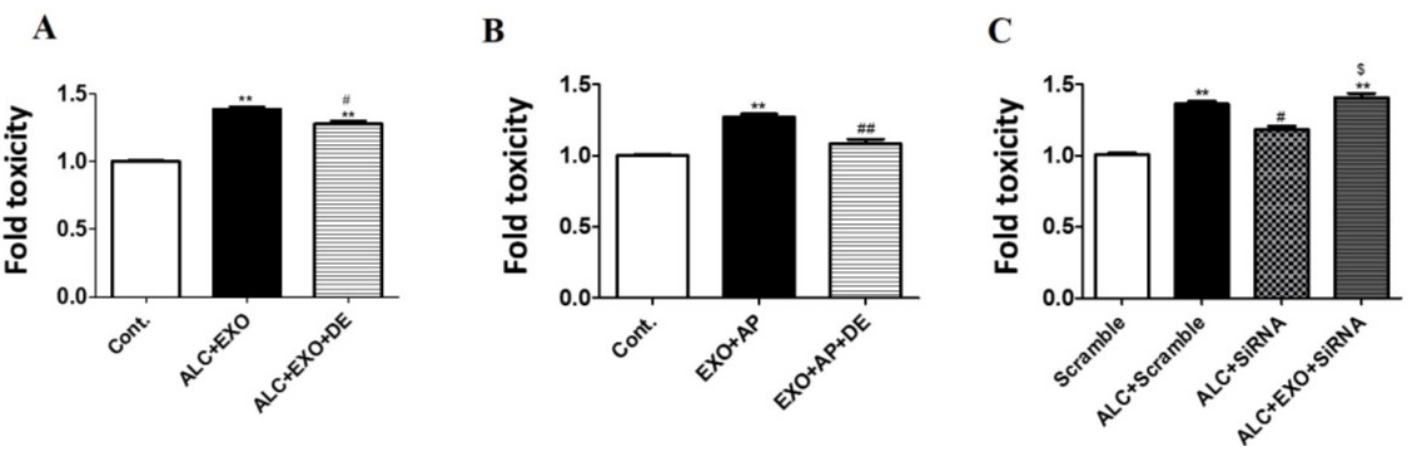

Figure 4-3. Rescue of exosomal CYP2E1 mediated toxicity by selective CYP2E1 inhibitor (DE) and CYP2E1 siRNA.

A-B) HepaRG cells were treated with human plasma exosomes \pm ALC/APAP with/without DE for 2 days. One-way ANOVA was used to measure statistical significance. * represents significance when compared to control, \# compared to EXO+ETH/APAP. C) HepaRG cells were treated with human plasma exosomes \pm ALC with/without CYP2E1 siRNA. Cytotoxicity was measured using LDH cell viability assay. The data shown here represent n\#3 experiments. One-way ANOVA with Tukey's multiple comparison test was used to measure statistical significance. \# represents significance when compared against scramble, $\$$ compared to ALC + scramble, $\wedge$ compared to ALC + si2E1. 
$\mathbf{a}$

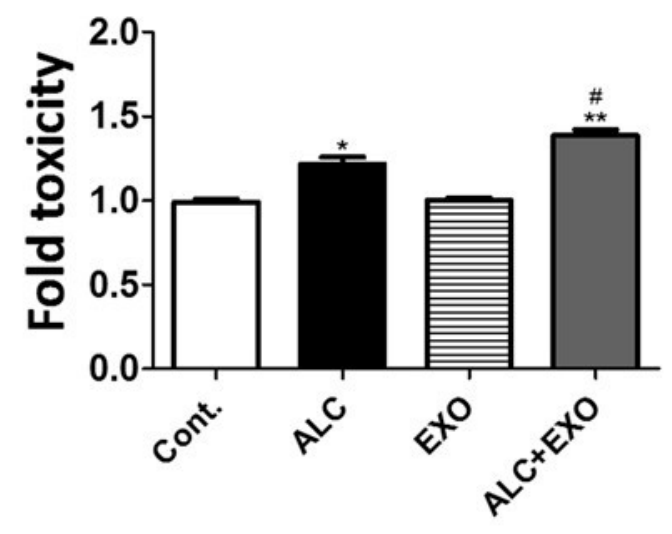

b

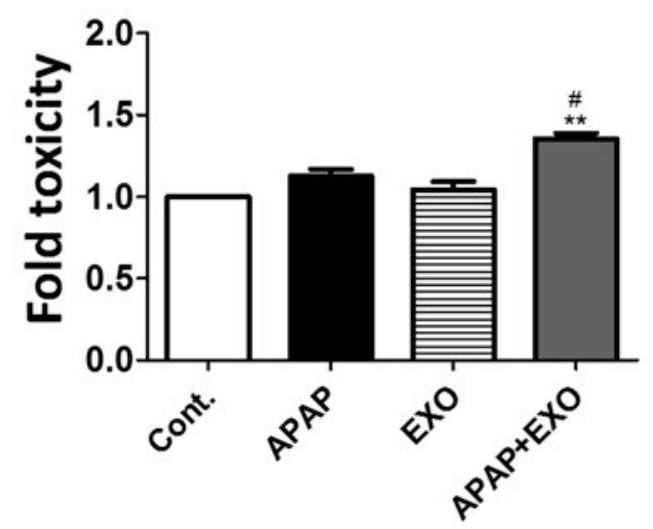

Figure 4-4. Effect of plasma exosomal CYP2E1 in U1 cells upon ethanol treatment.

Plasma exosomes were isolated from healthy human plasma and then treated to U1 cells +/- ALC/APAP for 2 days. Cytotoxicity was measured using LDH cell viability assay. The data shown here represent $n \# 3$ experiments. One-way ANOVA was used to measure statistical significance. * represents significance when compared to control, \# compared to ALC/APAP.

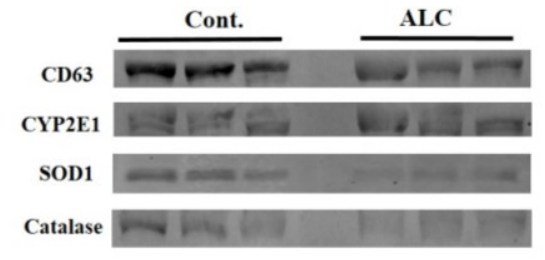

A

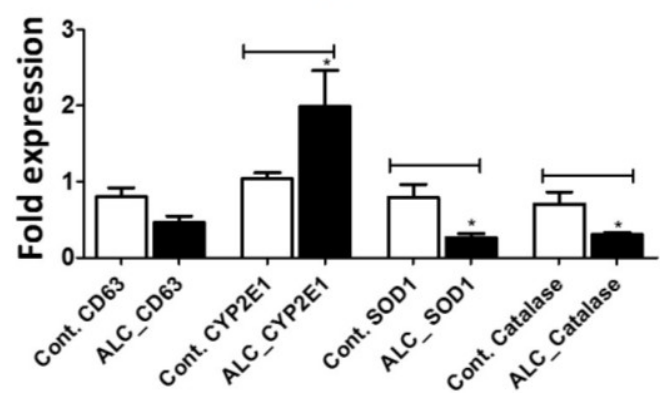

B

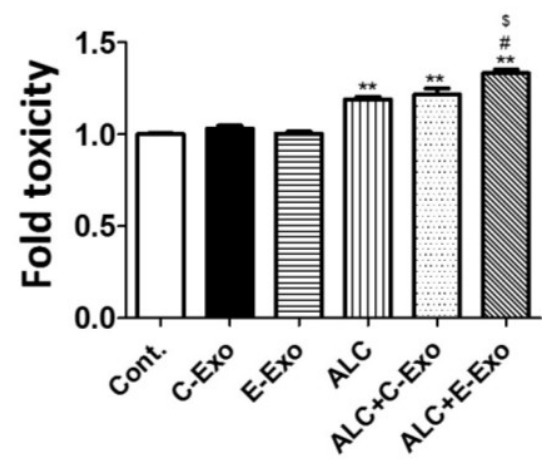

Figure 4-5. Effect of alcohol on murine plasma exosomal CYP2E1 and AOEs. A) Expression of CYP2E1, SOD1, and catalase in plasma exosomes in binge-drinking mice. B) Effect of exosomes derived from ALC fed mice on ALC-induced toxicity in U1 cells after 4 days. One-way ANOVA was used to measure statistical significance. * represents significance compared to control and control-exosomes (C-Exo), \# represents significance compared ALC induced exosomes (E-Exo), \$ represents significance relative to ALC and ALC+C-Exo. 


\section{Discussion}

CYP2E1, one of the key components of the microsomal ethanol oxidizing system (MEOS), is mainly involved in metabolism of small molecular xenobiotics [39]. CYP2E1 can activate procarcinogens and other toxic compounds found in cigarette smoke and various environmental pollutants [332-334]. However, the most clinically relevant role of CYP2E1 is associated with ALC and APAP metabolism, especially at their higher doses. Though liver is the primary site where CYP2E1 mediated metabolism exerts its most damaging effect, extra-hepatic tissues are often affected to a various degree as well [335, 336]. While the hepatic pathway of ALC- and APAP-induced toxicity is well established, the extra-hepatic mechanism(s) for ALC- and APAP-induced toxicity is not clearly defined. We recently reported that CYP2E1 is abundantly present in exosomes derived from healthy human plasma [48]. We also predicted that the majority of the CYP2E1 in plasma exosomes are derived from hepatic cells, along with some from extra-hepatic cells. Based on our previous findings, we hypothesized that the metabolically active plasma exosomal CYP2E1 cargo participates in cellular pathophysiology and contributes to ALC- and APAP-induced toxicity in hepatic and non-hepatic cells.

In our current study, we observed that plasma exosomes treatment additively/synergistically increased ALC- and APAP-induced toxicity in HepaRG hepatocytes and U1 monocytic cells. Further, using CYP2E1 specific inhibitor and CYP2E1 siRNA, we confirmed the role of CYP2E1 in exosomes induced toxicity. Besides, we found that alcohol exposure increases packaging of CYP2E1 in exosomes derived from plasma of mice. We also observed enhanced toxicity when these ALCinduced exosomes were treated to U1 cells, compared to treatment with exosomes derived from non-drinking mouse plasma. The results suggest that the exosomal cargos, specifically CYP2E1, may have far-reaching metabolic consequence in ALC- and APAPinduced pathogenesis in hepatic and extra-hepatic cells (Figure 4-6).

Exosomes are released by almost all cell types into their extracellular space or into biofluids such as plasma [337]. Recent studies on exosomes reveal that they can be actively involved in intercellular communication with both beneficial and harmful physiological consequences [325, 338-340]. Exosomes have been suggested as a potential biomarker in ALC and APAP associated pathologies. Several studies have reported circulating exosomal miRNAs as potential marker for ALC and other drug induced inflammatory liver diseases. For example, Momen-Heravi et al., (2015) showed that liver derived exosomal transfer of miR-122 rendered monocytes more sensitive to inflammation, which is a key phenomenon in AH pathogenesis [341]. They also showed that the number of exosomal production was increased and the ethanol concentration in the human sera was reduced after ALC consumption. Another study demonstrated that ALC mediated increased EV production and macrophage activation follows CYP2E1dependent pathway [177]. Similar studies have been conducted relating exosomal pathway and APAP-induced injury. Cho et al., (2018) showed that exosomes obtained from APAP-induced liver injury caused toxicity in the naïve recipient hepatocytes in a 

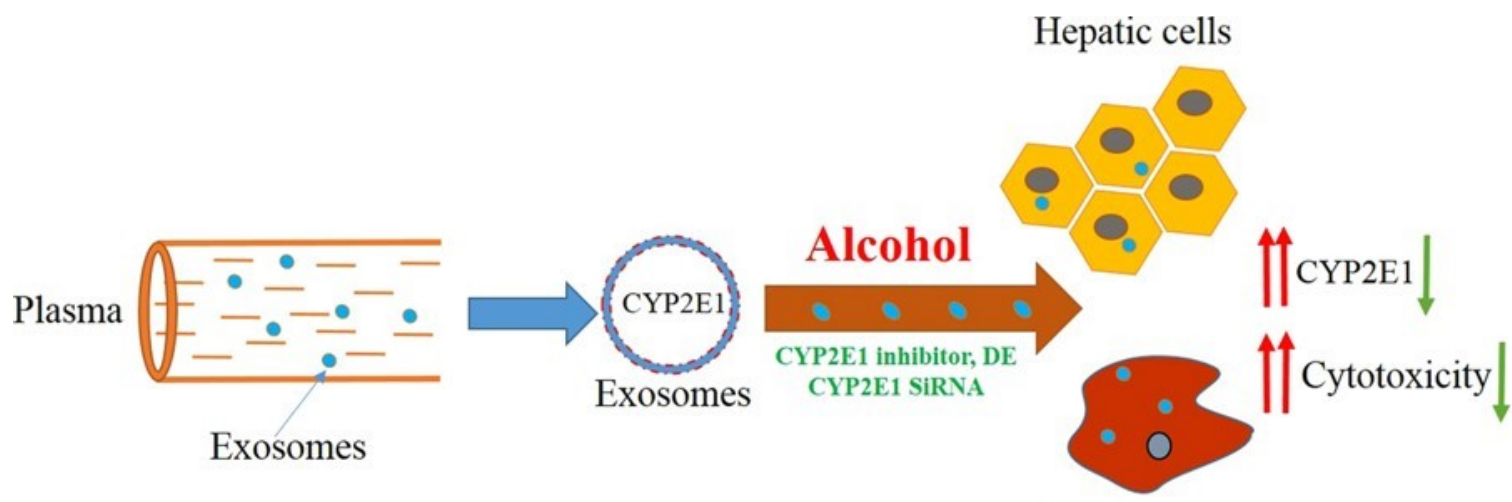

Monocytic cells

Figure 4-6. Schematic representation of the plasma exosomal CYP2E1 mediated toxicity.

Plasma derived exosomes can carry metabolically active CYP2E1 which may influence the metabolic pathway of ALC and APAP in hepatic and extra-hepatic cells resulting in increased cytotoxicity. 
mouse model [342]. Another study suggests that alteration in the hepatocytes derived exosome levels can act as an early predictor of liver injury in subtoxic APAP exposure [343]. These studies are shedding new light in the phenomenon of xenobiotics-induced toxicity and disease pathogenesis.

Plasma exosomes contain not only CYP enzymes but also many other macromolecules such as miRNAs [344]. Exosomal miRNAs, especially the mir200 family, have been hypothesized as a major target to combat oxidative stress-induced effects. CYP2E1-mediated increased oxidative stress in hepatocytes upon ALC exposure is expected to induce mir200s, which are packaged in exosomes, secreted, and circulated in the plasma [345]. The mir200s promote oxidative stress by disrupting the SIRT1/FOXO1/eNOS pathway and inhibiting transcription of antioxidant enzymes (AOEs), especially catalase [346]. A reduced level of AOEs compromises their ability to neutralize ROS, and thus contributes to enhanced oxidative stress. Dysregulation of mir200s and resultant oxidative stress cause apoptosis and cellular senescence in many cells [347]. Hence, exosomal mir200s- and CYP2E1-induced oxidative stress would enhance ALC-induced toxicity additively and/or synergistically. We have observed that plasma exosomes derived from alcohol drinker subjects contain significant level of mir-200c-3p compared to healthy subjects (unpublished observation).

Exosomal miRNAs, especially the mir200 family, have been hypothesized as a major target to combat oxidative stress-induced effects[348]. CYP2E1-mediated increased oxidative stress in hepatocytes upon ALC exposure is expected to induce mir200s, which are packaged in exosomes, secreted, and circulated in the plasma[345]. The mir200s promote oxidative stress by disrupting the SIRT1/FOXO1/eNOS pathway and inhibiting transcription of AOEs, especially catalase[346]. Dysregulation of mir-200s and resultant oxidative stress cause apoptosis and cellular senescence in many cells[347]. Hence, exosomal mir200s- and CYP2E1-induced oxidative stress would enhance ALCinduced toxicity additively and/or synergistically. We have observed that plasma exosomes derived from alcohol drinker subjects contain significant level of mir-200c-3p compared to healthy subjects (unpublished observation).

We observed a somewhat similar phenomenon when plasma exosomes from a binge-drinking mouse model were characterized for their AOE expression. The expression of CYP2E1 enzyme was significantly upregulated, while SOD1 and catalase were downregulated upon alcohol exposure in these mice. When these exosomes were treated or exposed to recipient U1 monocytic cells, the ALC-induced toxicity was further exacerbated. The overall increase in oxidative stress elements mediated by CYP2E1 in the ALC-induced exosomes may have acted as an underlying factor in the increased toxicity.

Through the findings of this study we suggest that plasma exosomes contribute to ALC/APAP-induced toxicity, via delivery of CYP2E1 to cells that have high cellular CYP2E1 (hepatic) as well as low cellular CYP2E1 (monocytic) levels. Upon further validation, the plasma exosomal CYP2E1 pathway may help to better understand ALC-, APAP-, and other xenobiotic-induced toxicity, especially in the extra-hepatic tissues. 
Plasma exosomes as "liquid biopsy" has been gaining attention in the drug metabolism and drug- drug interaction (DDI) arena of drug development [349]. Improvement of analytical techniques has enabled the quantification of many endogenous components in human biofluids such as plasma. Hence, plasma exosomal CYP2E1 load, along with other relevant CYPs, can be taken into consideration while studying DDI and pharmacokinetic profiling of new and existing drugs. This will not only help understanding drug-induced pathologies in the hepatic and extra-hepatic tissues but also in optimizing dosing regimens.

In conclusion, this study demonstrates that both human and murine plasma exosomal cargo, specifically CYP2E1, play a role in exacerbating ALC- and APAPinduced toxicity in hepatocytes and monocytes. Exosomal transport between cells can be either beneficial or harmful, and thus a better understanding of the roles of exosomes in drug abuse/overdose conditions is crucial. 


\section{CHAPTER 5. CONCLUSION AND FUTURE DIRECTIONS}

\section{Conclusion}

Despite having many therapeutic properties, the usage of DAS is restricted due to its own inherent toxicity. Therefore, there was a critical need for replacing DAS with a safer CYP2E1 inhibitor. In aim 1, we conducted a thorough analysis of the CYP2E1 inhibition kinetics of DAS and seven structural analogs. This study provided several DAS analogs that showed better inhibition characteristics than DAS. In aim 2, we observed that at least three DAS analogs- thiophene, allyl methyl sulfide, and diallyl ether appeared to be less toxic than DAS for the cellular models evaluated in this project. These compounds were also better than DAS with regard to rescuing ALC- and APAPinduced toxicity as well as reducing viral replication. Overall, the results are very encouraging in the search for a superior alternative to the widely used DAS as a selective CYP2E1 inhibitor as a research tool and/or as a possible combination/preventive therapy in ALC-, APAP-, and other xenobiotic-induced toxicity.

For the second project, we assumed that plasma exosomal CYP2E1 cargo could play critical role in xenobiotics-induced toxicity. In this project, we demonstrated that both human and murine plasma derived exosomal cargo, specifically CYP2E1, play a significant role in exacerbating ALC- and APAP-induced toxicity in hepatocytes and monocytes. By inhibiting cellular CYP2E1 by DE (one of the newly identified CYP2E1 inhibitors) as well by siRNA, we observed that plasma exosomes can enhance cytotoxicity by participating in the metabolism of ALC and APAP in both hepatic and non-hepatic cells.

\section{Future Directions}

Upon further analysis, the newly identified analogs may replace DAS as a research tool or potentially, as a therapeutic regimen for preventing CYP2E1-mediated xenobiotic- and disease-induced toxicity. The immediate next goal should be evaluating these analogs in in vivo systems using appropriate animal models to verify our in vitro findings. Despite its use in research for many years, the pharmacokinetic properties of DAS are not well characterized. Therefore, evaluation of the pharmacokinetic parameters of DAS and the new analogs using suitable in vitro and in vivo systems should be undertaken to better understand the pharmacokinetic/pharmacodynamic relationship of these compounds. Successful completion of these studies is required for the novel analogs to be considered as either a dietary supplement or as an adjuvant therapy in CYP2E1 mediated pathologies.

Since intra- /inter-cellular exosomal transport of biomolecules can be either beneficial or harmful, a better understanding of the roles of exosomes in drug abuse/overdose conditions is crucial. Hence, further studies, especially using human plasma exosomes from alcohol drinkers and acetaminophen abuse/overdose patient, are 
required to explore the full potential of exosomal CYP2E1 cargo and other CYP enzymes, as biochemical mediators in ALC and APAP metabolism and resulting toxicity. CYP2E1 in plasma exosomes may translocate to the brain by crossing the BBB and can contribute to the metabolic pathway of the CNS, an area which requires significant investigation. Using in vitro and in vivo studies, it is crucial to check the uptake of these exosomal CYP2E1 cargo into the brain and their probable role in ALC and APAP metabolism-induced neurodegenerative effects. Moreover, exosomes can be engineered to carry small molecular pharmaceutical drugs as well as large biomolecules such as proteins. The newly identified safer DAS analogs can be packaged in exosomes and thus, can be used for inhibiting CYP2E1 mediated metabolism of ALC and APAP in the brain. Moreover, exosomes can be manipulated to carry antioxidant enzymes to be delivered to the CNS which can help alleviate oxidative stress induced neurodegeneration. These are some exciting therapeutic intervention strategies which require in depth research by using appropriate in vitro and in vivo model. Additionally, the roles of other prevalent CYP enzymes in the plasma exosomes needs further attention. CYP3A4 is responsible for metabolism of $\sim 50 \%$ of the pharmaceutical drugs [350]. Hence, it is important to look into the contribution of plasma exosomal CYP3A4 as well as other drug metabolizing enzymes in relevant xenobiotic- and diseases-induced pathologies. 


\section{LIST OF REFERENCES}

1. Garcia-Suastegui, W.A., et al., The Role of CYP2E1 in the Drug Metabolism or Bioactivation in the Brain. Oxid Med Cell Longev, 2017. 2017: p. 4680732.

2. Zanger, U.M. and M. Schwab, Cytochrome P450 enzymes in drug metabolism: regulation of gene expression, enzyme activities, and impact of genetic variation. Pharmacol Ther, 2013. 138(1): p. 103-41.

3. Hedlund, E., J.A. Gustafsson, and M. Warner, Cytochrome P450 in the brain; a review. Curr Drug Metab, 2001. 2(3): p. 245-63.

4. Hukkanen, J., et al., Expression and regulation of xenobiotic-metabolizing cytochrome P450 (CYP) enzymes in human lung. Crit Rev Toxicol, 2002. 32(5): p. 391-411.

5. Nguyen, L.T., et al., Detection of cytochrome P450 and other drug-metabolizing enzyme mRNAs in peripheral blood mononuclear cells using DNA arrays. Drug Metab Dispos, 2000. 28(8): p. 987-93.

6. Rao, P., et al., Effects of Cigarette Smoke Condensate on Oxidative Stress, Apoptotic Cell Death, and HIV Replication in Human Monocytic Cells. PLoS One, 2016. 11(5): p. e0155791.

7. Xu, J., et al., The role of human cytochrome P450 $2 E 1$ in liver inflammation and fibrosis. Hepatology Communications, 2017. 1(10): p. 1043-1057.

8. Cederbaum, A.I., Alcohol metabolism. Clin Liver Dis, 2012. 16(4): p. 667-85.

9. Gonzalez, F.J., The 2006 Bernard B. Brodie Award Lecture. Cyp2e1. Drug Metab Dispos, 2007. 35(1): p. 1-8.

10. Ghanayem, B.I. and U. Hoffler, Investigation of xenobiotics metabolism, genotoxicity, and carcinogenicity using Cyp2e1(-/-) mice. Curr Drug Metab, 2007. 8(7): p. 728-49.

11. Bolt, H.M., P.H. Roos, and R. Thier, The cytochrome P-450 isoenzyme CYP2E1 in the biological processing of industrial chemicals: consequences for occupational and environmental medicine. Int Arch Occup Environ Health, 2003. 76(3): p. 174-85.

12. Krekels, E.H.J., et al., Chapter 8 - Hepatic Drug Metabolism in Pediatric Patients, in Drug Metabolism in Diseases, W. Xie, Editor. 2017, Academic Press: Boston. p. 181-206.

13. Oneta, C.M., et al., Dynamics of cytochrome P4502E1 activity in man: induction by ethanol and disappearance during withdrawal phase. J Hepatol, 2002. 36(1): p. 47-52.

14. Cederbaum, A.I., Role of CYP2E1 in ethanol-induced oxidant stress, fatty liver and hepatotoxicity. Dig Dis, 2010. 28(6): p. 802-11.

15. Aubert, J., et al., Increased expression of cytochrome P450 $2 E 1$ in nonalcoholic fatty liver disease: mechanisms and pathophysiological role. Clin Res Hepatol Gastroenterol, 2011. 35(10): p. 630-7.

16. Leung, T., et al., Cytochrome P450 2E1 (CYP2E1) regulates the response to oxidative stress and migration of breast cancer cells. Breast Cancer Research, 2013. 15(6): p. R107. 
17. Morgan, E.T., Regulation of cytochrome 450 by inflammatory mediators: why and how? Drug Metab Dispos, 2001. 29(3): p. 207-12.

18. WHO global status report on alcohol and health 2018.

19. Heit, C., et al., The role of CYP2E1 in alcohol metabolism and sensitivity in the central nervous system. Subcell Biochem, 2013. 67: p. 235-47.

20. Teschke, R., Alcoholic Liver Disease: Alcohol Metabolism, Cascade of Molecular Mechanisms, Cellular Targets, and Clinical Aspects. Biomedicines, 2018. 6(4).

21. Dunn, W. and V.H. Shah, Pathogenesis of Alcoholic Liver Disease. Clin Liver Dis, 2016. 20(3): p. 445-56.

22. Stickel, F., et al., Pathophysiology and Management of Alcoholic Liver Disease: Update 2016. Gut Liver, 2017. 11(2): p. 173-188.

23. Heit, C., et al., The role of CYP2E1 in alcohol metabolism and sensitivity in the central nervous system. Sub-cellular biochemistry, 2013. 67: p. 235-247.

24. Zakhari, S., Overview: how is alcohol metabolized by the body? Alcohol Res Health, 2006. 29(4): p. 245-54.

25. Connor, J., Alcohol consumption as a cause of cancer. Addiction, 2017. 112(2): p. 222-228.

26. Monnig, M.A., Immune activation and neuroinflammation in alcohol use and HIV infection: evidence for shared mechanisms. Am J Drug Alcohol Abuse, 2017. 43(1): p. 7-23.

27. Rehm, J., et al., The association between alcohol use, alcohol use disorders and tuberculosis (TB). A systematic review. BMC Public Health, 2009. 9: p. 450.

28. Blieden, M., et al., A perspective on the epidemiology of acetaminophen exposure and toxicity in the United States. Expert Rev Clin Pharmacol, 2014. 7(3): p. 3418.

29. Lee, W.M., Acetaminophen (APAP) hepatotoxicity-Isn't it time for APAP to go away? Journal of hepatology, 2017. 67(6): p. 1324-1331.

30. Yoon, E., et al., Acetaminophen-Induced Hepatotoxicity: a Comprehensive Update. Journal of clinical and translational hepatology, 2016. 4(2): p. 131-142.

31. Administration, U.F.a.D., Recommended Warning for Overthe-Counter AcetaminophenContaining Drug Products and Labeling Statements Regarding Serious Skin Reactions. 2017.

32. Ghanem, C.I., et al., Acetaminophen from liver to brain: New insights into drug pharmacological action and toxicity. Pharmacological research, 2016. 109: p. 119-131.

33. Blantz, R.C., Acetaminophen: acute and chronic effects on renal function. Am J Kidney Dis, 1996. 28(1 Suppl 1): p. S3-6.

34. Cederbaum, A.I., ALCOHOL METABOLISM. Clinics in liver disease, 2012. 16(4): p. 667-685.

35. Ceni, E., T. Mello, and A. Galli, Pathogenesis of alcoholic liver disease: role of oxidative metabolism. World journal of gastroenterology, 2014. 20(47): p. 1775617772.

36. Lieber, C.S., The discovery of the microsomal ethanol oxidizing system and its physiologic and pathologic role. Drug Metab Rev, 2004. 36(3-4): p. 511-29.

37. Roberts, B.J., et al., Induction of CYP2E1 in liver, kidney, brain and intestine during chronic ethanol administration and withdrawal: evidence that CYP2E1 
possesses a rapid phase half-life of 6 hours or less. Biochem Biophys Res Commun, 1994. 205(2): p. 1064-71.

38. Roberts, B.J., et al., Ethanol induces CYP2E1 by protein stabilization. Role of ubiquitin conjugation in the rapid degradation of CYP2E1. J Biol Chem, 1995. 270(50): p. 29632-5.

39. Lu, Y. and A.I. Cederbaum, CYP2E1 and Oxidative Liver Injury by Alcohol. Free radical biology \& medicine, 2008. 44(5): p. 723-738.

40. Jin, M., et al., Regulation of cytochrome P450 2el expression by ethanol: role of oxidative stress-mediated pkc/jnk/sp1 pathway. Cell Death \&Amp; Disease, 2013. 4: p. e554.

41. Vasiliou, V., et al., CYP2E1 and catalase influence ethanol sensitivity in the central nervous system. Pharmacogenet Genomics, 2006. 16(1): p. 51-8.

42. Warner, M. and J.A. Gustafsson, Effect of ethanol on cytochrome P450 in the rat brain. Proc Natl Acad Sci U S A, 1994. 91(3): p. 1019-23.

43. Gill, K., et al., Enzymatic production of acetaldehyde from ethanol in rat brain tissue. Alcohol Clin Exp Res, 1992. 16(5): p. 910-5.

44. Aragon, C.M., F. Rogan, and Z. Amit, Ethanol metabolism in rat brain homogenates by a catalase-H2O2 system. Biochem Pharmacol, 1992. 44(1): p. 93-8.

45. Singh, A.K., et al., Effects of chronic ethanol drinking on the blood-brain barrier and ensuing neuronal toxicity in alcohol-preferring rats subjected to intraperitoneal LPS injection. Alcohol and Alcoholism, 2007. 42(5): p. 385-399.

46. Cho, Y.-E., et al., Increased ethanol-inducible cytochrome P450-2E1 and cytochrome P450 isoforms in exosomes of alcohol-exposed rodents and patients with alcoholism through oxidative and endoplasmic reticulum stress. Hepatology communications, 2017. 1(7): p. 675-690.

47. Kodidela, S., et al., Cytokine profiling of exosomes derived from the plasma of HIV-infected alcohol drinkers and cigarette smokers. PloS one, 2018. 13(7): p. e0201144-e0201144.

48. Kumar, S., et al., Specific packaging and circulation of cytochromes P450, especially 2E1 isozyme, in human plasma exosomes and their implications in cellular communications. Biochem Biophys Res Commun, 2017. 491(3): p. 675680.

49. Lieber, C.S., Cytochrome P-4502E1: its physiological and pathological role. Physiological Reviews, 1997. 77(2): p. 517-544.

50. James, L., J.E. Sullivan, and D. Roberts, The proper use of acetaminophen. Paediatrics \& child health, 2011. 16(9): p. 544-547.

51. Mazaleuskaya, L.L., et al., PharmGKB summary: pathways of acetaminophen metabolism at the therapeutic versus toxic doses. Pharmacogenetics and genomics, 2015. 25(8): p. 416-426.

52. Mitchell, J.R., et al., Acetaminophen-induced hepatic necrosis. IV. Protective role of glutathione. J Pharmacol Exp Ther, 1973. 187(1): p. 211-7.

53. Gillette, J.R., et al., Formation of chemically reactive metabolites of phenacetin and acetaminophen. Adv Exp Med Biol, 1981. 136 Pt B: p. 931-50.

54. Jaeschke, H., Acetaminophen: Dose-Dependent Drug Hepatotoxicity and Acute Liver Failure in Patients. Dig Dis, 2015. 33(4): p. 464-71. 
55. Hu, Y., et al., Chlormethiazole as an efficient inhibitor of cytochrome P450 2E1 expression in rat liver. J Pharmacol Exp Ther, 1994. 269(3): p. 1286-91.

56. Gonzalez, F.J., CYP2E1. Drug Metabolism and Disposition, 2007. 35(1): p. 1-8.

57. Rao, P.S.S., et al., Diallyl Sulfide: Potential Use in Novel Therapeutic Interventions in Alcohol, Drugs, and Disease Mediated Cellular Toxicity by Targeting Cytochrome P450 2E1. Current drug metabolism, 2015. 16(6): p. 486503.

58. Zand, R., et al., Inhibition and induction of cytochrome P4502E1-catalyzed oxidation by isoniazid in humans. Clin Pharmacol Ther, 1993. 54(2): p. 142-9.

59. Buhler, R., et al., Perivenous expression of ethanol-inducible cytochrome P450 IIE1 in livers from alcoholics and chronically ethanol-fed rats. Alcohol Alcohol Suppl, 1991. 1: p. 311-5.

60. Abdelmegeed, M.A., et al., Role of CYP2E1 in Mitochondrial Dysfunction and Hepatic Injury by Alcohol and Non-Alcoholic Substances. Current molecular pharmacology, 2017. 10(3): p. 207-225.

61. Caro, A.A. and A.I. Cederbaum, Oxidative stress, toxicology, and pharmacology of CYP2E1. Annu Rev Pharmacol Toxicol, 2004. 44: p. 27-42.

62. Gorsky, L.D., D.R. Koop, and M.J. Coon, On the stoichiometry of the oxidase and monooxygenase reactions catalyzed by liver microsomal cytochrome P-450.

Products of oxygen reduction. J Biol Chem, 1984. 259(11): p. 6812-7.

63. Knecht, K.T., et al., Free radical adducts in the bile of rats treated chronically with intragastric alcohol: inhibition by destruction of Kupffer cells. Mol Pharmacol, 1995. 47(5): p. 1028-34.

64. Tsukamoto, H. and S.C. Lu, Current concepts in the pathogenesis of alcoholic liver injury. Faseb j, 2001. 15(8): p. 1335-49.

65. Nanji, A.A., et al., Markedly enhanced cytochrome P450 2E1 induction and lipid peroxidation is associated with severe liver injury in fish oil-ethanol-fed rats. Alcohol Clin Exp Res, 1994. 18(5): p. 1280-5.

66. Morimoto, M., et al., Role of cytochrome P4502E1 in alcoholic liver disease pathogenesis. Alcohol, 1993. 10(6): p. 459-64.

67. Gouillon, Z., et al., Inhibition of ethanol-induced liver disease in the intragastric feeding rat model by chlormethiazole. Proc Soc Exp Biol Med, 2000. 224(4): p. 302-8.

68. Aleynik, M.K., et al., Polyenylphosphatidylcholine opposes the increase of cytochrome P-4502E1 by ethanol and corrects its iron-induced decrease. Alcohol Clin Exp Res, 1999. 23(1): p. 96-100.

69. Guan, M.J., et al., Hepatoprotective effects of garlic against ethanol-induced liver injury: A mini-review. Food Chem Toxicol, 2018. 111: p. 467-473.

70. Li, S., et al., The Role of Oxidative Stress and Antioxidants in Liver Diseases. Int J Mol Sci, 2015. 16(11): p. 26087-124.

71. Aljomah, G., et al., Induction of CYP2E1 in non-alcoholic fatty liver diseases. Experimental and molecular pathology, 2015.99(3): p. 677-681.

72. Leung, T.-M. and N. Nieto, CYP2E1 and oxidant stress in alcoholic and nonalcoholic fatty liver disease. Journal of Hepatology, 2013. 58(2): p. 395-398. 
73. Emery, M.G., et al., CYP2E1 activity before and after weight loss in morbidly obese subjects with nonalcoholic fatty liver disease. Hepatology, 2003. 38(2): p. 428-35.

74. van Rongen, A., et al., Morbidly Obese Patients Exhibit Increased CYP2E1Mediated Oxidation of Acetaminophen. Clinical pharmacokinetics, 2016. 55(7): p. 833-847.

75. Swaminathan, K., et al., Inhibition of CYP2E1 leads to decreased advanced glycated end product formation in high glucose treated ADH and CYP2E1 overexpressing VL-17A cells. Biochim Biophys Acta, 2013. 1830(10): p. 4407-16.

76. Abdelmegeed, M.A., et al., Critical role of cytochrome P450 2E1 (CYP2E1) in the development of high fat-induced non-alcoholic steatohepatitis. Journal of hepatology, 2012. 57(4): p. 860-866.

77. Dai, N., et al., Antioxidant properties of proanthocyanidins attenuate carbon tetrachloride (CCl4)-induced steatosis and liver injury in rats via CYP2E1 regulation. Journal of medicinal food, 2014. 17(6): p. 663-669.

78. Sun, Q., et al., Roles of CYP2e1 in 1,2-dichloroethane-induced liver damage in mice. Environ Toxicol, 2016. 31(11): p. 1430-1438.

79. Dey, A., Cytochrome P450 2E1: Its Role in Disease and Drug Metabolism. Subcellular Biochemistry, 2013. 67.

80. Sun, A.Y. and G.Y. Sun, Ethanol and oxidative mechanisms in the brain. J Biomed Sci, 2001. 8(1): p. 37-43.

81. Haorah, J., et al., Mechanism of alcohol-induced oxidative stress and neuronal injury. Free radical biology \& medicine, 2008. 45(11): p. 1542-1550.

82. Haorah, J., et al., Alcohol-induced oxidative stress in brain endothelial cells causes blood-brain barrier dysfunction. J Leukoc Biol, 2005. 78(6): p. 1223-32.

83. Haorah, J., et al., Oxidative stress activates protein tyrosine kinase and matrix metalloproteinases leading to blood-brain barrier dysfunction. J Neurochem, 2007. 101(2): p. 566-76.

84. Haorah, J., et al., Ethanol-induced activation of myosin light chain kinase leads to dysfunction of tight junctions and blood-brain barrier compromise. Alcohol Clin Exp Res, 2005. 29(6): p. 999-1009.

85. Shahabi, H.N., et al., Cytochrome P450 2E1 gene polymorphisms/haplotypes and Parkinson's disease in a Swedish population. J Neural Transm (Vienna), 2009. 116(5): p. 567-73.

86. Kiyohara, C., et al., APOE and CYP2E1 polymorphisms, alcohol consumption, and Parkinson's disease in a Japanese population. J Neural Transm (Vienna), 2011. 118(9): p. 1335-44.

87. Wu, R.M., et al., Genetic polymorphism of the CYP2E1 gene and susceptibility to Parkinson's disease in Taiwanese. J Neural Transm (Vienna), 2002. 109(11): p. 1403-14.

88. Leung, T., et al., Cytochrome P450 $2 E 1$ (CYP2E1) regulates the response to oxidative stress and migration of breast cancer cells. Breast Cancer Res, 2013. 15(6): p. R107.

89. Wang, Y., et al., Association between CYP2E1 genetic polymorphisms and lung cancer risk: a meta-analysis. Eur J Cancer, 2010. 46(4): p. 758-64. 
90. Jiang, O., et al., CYP2E1 polymorphisms and colorectal cancer risk: a HuGE systematic review and meta-analysis. Tumour Biol, 2013. 34(2): p. 1215-24.

91. Seitz, H.K. and X.D. Wang, The role of cytochrome P450 2 E1 in ethanolmediated carcinogenesis. Subcell Biochem, 2013. 67: p. 131-43.

92. Trafalis, D.T., et al., CYP2E1 and risk of chemically mediated cancers. Expert Opin Drug Metab Toxicol, 2010. 6(3): p. 307-19.

93. Sies, H., Oxidative stress: a concept in redox biology and medicine. Redox Biol, 2015. 4: p. 180-3.

94. Lugrin, J., et al., The role of oxidative stress during inflammatory processes. Biol Chem, 2014. 395(2): p. 203-30.

95. Jiang, T., Q. Sun, and S. Chen, Oxidative stress: A major pathogenesis and potential therapeutic target of antioxidative agents in Parkinson's disease and Alzheimer's disease. Prog Neurobiol, 2016. 147: p. 1-19.

96. Ivanov, A.V., et al., Oxidative Stress during HIV Infection: Mechanisms and Consequences. Oxidative medicine and cellular longevity, 2016. 2016: p. 8910396-8910396.

97. El-Amine, R., et al., HIV-1 Tat protein induces DNA damage in human peripheral blood B-lymphocytes via mitochondrial ROS production. Redox biology, 2017. 15: p. 97-108.

98. Price, T.O., et al., HIV-1 viral proteins gp120 and Tat induce oxidative stress in brain endothelial cells. Brain Res, 2005. 1045(1-2): p. 57-63.

99. Yew, W.W., et al., Does oxidative stress contribute to adverse outcomes in HIVassociated TB? J Antimicrob Chemother, 2018. 73(5): p. 1117-1120.

100. Rajopadhye, S.H., et al., Oxidative Stress Markers in Tuberculosis and HIV/TB Co-Infection. J Clin Diagn Res, 2017. 11(8): p. Bc24-bc28.

101. Suarez, G.V., et al., Immunoendocrine interactions during HIV-TB coinfection: implications for the design of new adjuvant therapies. Biomed Res Int, 2015. 2015: p. 461093.

102. Hu, J., et al., Effects of combined alcohol and anti-HIV drugs on cellular stress responses in primary hepatocytes and hepatic stellate and kupffer cells. Alcoholism, clinical and experimental research, 2015. 39(1): p. 11-20.

103. Williams, E.C., et al., Alcohol Use and Human Immunodeficiency Virus (HIV) Infection: Current Knowledge, Implications, and Future Directions. Alcoholism, clinical and experimental research, 2016. 40(10): p. 2056-2072.

104. Persidsky, Y., et al., HIV-1 infection and alcohol abuse: neurocognitive impairment, mechanisms of neurodegeneration and therapeutic interventions. Brain Behav Immun, 2011. 25 Suppl 1: p. S61-70.

105. Sherman, K.E., D. Thomas, and R.T. Chung, Human immunodeficiency virus and liver disease forum 2012. Hepatology, 2014. 59(1): p. 307-17.

106. Shin, D.-H., et al., Relationship of Oxidative Stress with HIV Disease Progression in HIV/HCV Co-infected and HIV Mono-infected Adults in Miami. International journal of bioscience, biochemistry and bioinformatics, 2012. 2(3): p. 217-223.

107. Ghosh, A.K., A. Sarkar, and H. Mitsuya, HIV-Associated Neurocognitive Disorder (HAND) and the Prospect of Brain-Penetrating Protease Inhibitors for Antiretroviral Treatment. Medical research archives, 2017. 5(4): p. 1113. 
108. Saylor, D., et al., HIV-associated neurocognitive disorder--pathogenesis and prospects for treatment. Nature reviews. Neurology, 2016. 12(4): p. 234-248.

109. Meyerhoff, D.J., Effects of alcohol and HIV infection on the central nervous system. Alcohol Res Health, 2001. 25(4): p. 288-98.

110. Silverstein, P.S., S. Kumar, and A. Kumar, HIV-1, HCV and alcohol in the CNS: potential interactions and effects on neuroinflammation. Current HIV research, 2014. 12(4): p. 282-292.

111. Zhou, L. and N.K. Saksena, HIV Associated Neurocognitive Disorders. Infectious disease reports, 2013. 5(Suppl 1): p. e8-e8.

112. Louboutin, J.-P. and D. Strayer, Role of Oxidative Stress in HIV-1-Associated Neurocognitive Disorder and Protection by Gene Delivery of Antioxidant Enzymes. Antioxidants (Basel, Switzerland), 2014. 3(4): p. 770-797.

113. Turchan, J., et al., Oxidative stress in HIV demented patients and protection ex vivo with novel antioxidants. Neurology, 2003. 60(2): p. 307-14.

114. Floreani, N.A., et al., Alcohol-induced interactive phosphorylation of Src and tolllike receptor regulates the secretion of inflammatory mediators by human astrocytes. J Neuroimmune Pharmacol, 2010. 5(4): p. 533-45.

115. Jin, M., et al., Regulation of cytochrome P450 2el expression by ethanol: role of oxidative stress-mediated pkc/jnk/sp1 pathway. Cell Death Dis, 2013. 4: p. e554.

116. Jin, M., et al., Effect of alcohol on drug efflux protein and drug metabolic enzymes in U937 macrophages. Alcohol Clin Exp Res, 2011. 35(1): p. 132-9.

117. Lu, Y. and A.I. Cederbaum, Cytochrome P450s and Alcoholic Liver Disease. Current pharmaceutical design, 2018. 24(14): p. 1502-1517.

118. Ande, A., et al., Enhanced oxidative stress by alcohol use in $\mathrm{HIV}+$ patients: possible involvement of cytochrome P450 $2 E 1$ and antioxidant enzymes. AIDS research and therapy, 2015. 12: p. 29-29.

119. Gong, Y., et al., The role of cytochrome P450 $2 E 1$ on ethanol-mediated oxidative stress and HIV replication in human monocyte-derived macrophages. Biochemistry and biophysics reports, 2018. 17: p. 65-70.

120. Rao, P.S., et al., Diallyl Sulfide: Potential Use in Novel Therapeutic Interventions in Alcohol, Drugs, and Disease Mediated Cellular Toxicity by Targeting Cytochrome P450 2E1. Curr Drug Metab, 2015. 16(6): p. 486-503.

121. Iciek, M., I. Kwiecien, and L. Wlodek, Biological properties of garlic and garlicderived organosulfur compounds. Environ Mol Mutagen, 2009. 50(3): p. 247-65.

122. Rai, S.K., M. Sharma, and M. Tiwari, Inhibitory effect of novel diallyldisulfide analogs on HMG-CoA reductase expression in hypercholesterolemic rats: CREB as a potential upstream target. Life Sci, 2009. 85(5-6): p. 211-9.

123. Wang, H.C., et al., Molecular mechanisms of garlic-derived allyl sulfides in the inhibition of skin cancer progression. Ann N Y Acad Sci, 2012. 1271: p. 44-52.

124. Kalayarasan, S., et al., Diallyl sulfide enhances antioxidants and inhibits inflammation through the activation of Nrf2 against gentamicin-induced nephrotoxicity in Wistar rats. Eur J Pharmacol, 2009. 606(1-3): p. 162-71.

125. Ho, C.Y., et al., Effect of diallyl sulfide on in vitro and in vivo Nrf2-mediated pulmonic antioxidant enzyme expression via activation ERK/p38 signaling pathway. J Agric Food Chem, 2012. 60(1): p. 100-7. 
126. Zitka, O., et al., Redox status expressed as GSH: GSSG ratio as a marker for oxidative stress in paediatric tumour patients. Oncology letters, 2012. 4(6): p. 1247-1253.

127. Ho, C.Y., et al., Diallyl sulfide as a potential dietary agent to reduce TNF-alphaand histamine-induced proinflammatory responses in A7r5 cells. Mol Nutr Food Res, 2014. 58(5): p. 1069-78.

128. Kalayarasan, S., N. Sriram, and G. Sudhandiran, Diallyl sulfide attenuates bleomycin-induced pulmonary fibrosis: critical role of iNOS, NF-kappaB, TNFalpha and IL-1beta. Life Sci, 2008. 82(23-24): p. 1142-53.

129. Lee, H.S., et al., Inhibition of cyclooxygenase 2 expression by diallyl sulfide on joint inflammation induced by urate crystal and IL-1beta. Osteoarthritis Cartilage, 2009. 17(1): p. 91-9.

130. Guyonnet, D., et al., Mechanisms of protection against aflatoxin B(1) genotoxicity in rats treated by organosulfur compounds from garlic. Carcinogenesis, 2002. 23(8): p. 1335-41.

131. Guyonnet, D., et al., Antimutagenic activity of organosulfur compounds from Allium is associated with phase II enzyme induction. Mutat Res, 2001. 495(1-2): p. 135-45.

132. Liu, L.G., et al., CYP2E1-dependent hepatotoxicity and oxidative damage after ethanol administration in human primary hepatocytes. World J Gastroenterol, 2005. 11(29): p. 4530-5.

133. Morimoto, M., et al., Modulation of experimental alcohol-induced liver disease by cytochrome P450 2 E1 inhibitors. Hepatology, 1995. 21(6): p. 1610-7.

134. Morimoto, M., et al., CYP-2E1 inhibitors partially ameliorate the changes in hepatic fatty acid composition induced in rats by chronic administration of ethanol and a high fat diet. J Nutr, 1995. 125(12): p. 2953-64.

135. Swaminathan, K., D.L. Clemens, and A. Dey, Inhibition of CYP2E1 leads to decreased malondialdehyde-acetaldehyde adduct formation in VL-17A cells under chronic alcohol exposure. Life sciences, 2013. 92(6-7): p. 325-336.

136. Zimatkin, S.M., et al., Enzymatic mechanisms of ethanol oxidation in the brain. Alcohol Clin Exp Res, 2006. 30(9): p. 1500-5.

137. Moore, N.D., E. Gulmez, and P. Blin, Is paracetamol hepatotoxic at normal doses? Bmj, 2013. 346: p. f1519.

138. Hu, J.J., et al., Protective effects of diallyl sulfide on acetaminophen-induced toxicities. Food Chem Toxicol, 1996. 34(10): p. 963-9.

139. Dai, Y. and A.I. Cederbaum, Cytotoxicity of acetaminophen in human cytochrome P4502E1-transfected HepG2 cells. J Pharmacol Exp Ther, 1995. 273(3): p. 1497505.

140. Wu, D. and A.I. Cederbaum, Cyclosporine A protects against arachidonic acid toxicity in rat hepatocytes: role of CYP2E1 and mitochondria. Hepatology, 2002. 35(6): p. 1420-30.

141. Shah, A., et al., HIV gp120- and methamphetamine-mediated oxidative stress induces astrocyte apoptosis via cytochrome P450 2E1. Cell Death Dis, 2013. 4: p. e850.

142. Jin, L. and T.A. Baillie, Metabolism of the chemoprotective agent diallyl sulfide to glutathione conjugates in rats. Chem Res Toxicol, 1997. 10(3): p. 318-27. 
143. Brady, J.F., et al., Inhibition of cytochrome P-450 $2 E 1$ by diallyl sulfide and its metabolites. Chem Res Toxicol, 1991. 4(6): p. 642-7.

144. WHO, Global status report on alcohol and health - 2014. 2014.

145. Rusyn, I. and R. Bataller, Alcohol and toxicity. J Hepatol, 2013. 59(2): p. 387-8.

146. Seitz, H.K. and P. Becker, Alcohol metabolism and cancer risk. Alcohol Res Health, 2007. 30(1): p. 38-41, 44-7.

147. Chan, C. and J. Levitsky, Infection and Alcoholic Liver Disease. Clin Liver Dis, 2016. 20(3): p. 595-606.

148. Chargaff, E. and R. West, The biological significance of the thromboplastic protein of blood. J Biol Chem, 1946. 166(1): p. 189-97.

149. Wolf, $\mathrm{P} .$, The nature and significance of platelet products in human plasma. $\mathrm{Br} \mathrm{J}$ Haematol, 1967. 13(3): p. 269-88.

150. Todorova, D., et al., Extracellular Vesicles in Angiogenesis. Circ Res, 2017. 120(10): p. 1658-1673.

151. Kowal, J., M. Tkach, and C. Théry, Biogenesis and secretion of exosomes. Current Opinion in Cell Biology, 2014. 29: p. 116-125.

152. Karpman, D., A.-1. Ståhl, and I. Arvidsson, Extracellular vesicles in renal disease. Nature Reviews Nephrology, 2017. 13: p. 545.

153. Akers, J.C., et al., Biogenesis of extracellular vesicles (EV): exosomes, microvesicles, retrovirus-like vesicles, and apoptotic bodies. Journal of neurooncology, 2013. 113(1): p. 1-11.

154. Yáñez-Mó, M., et al., Biological properties of extracellular vesicles and their physiological functions. Journal of extracellular vesicles, 2015. 4: p. 2706627066.

155. M, H.R., et al., Exosomes: From Garbage Bins to Promising Therapeutic Targets. Int J Mol Sci, 2017. 18(3).

156. Johnstone, R.M., Revisiting the road to the discovery of exosomes. Blood Cells Mol Dis, 2005. 34(3): p. 214-9.

157. Johnstone, R.M., et al., Vesicle formation during reticulocyte maturation. Association of plasma membrane activities with released vesicles (exosomes). J Biol Chem, 1987. 262(19): p. 9412-20.

158. Xiao, D., et al., Identifying $m R N A$, MicroRNA and Protein Profiles of Melanoma Exosomes. PLoS ONE, 2012. 7(10): p. e46874.

159. Li, S., et al., exoRBase: a database of circRNA, lncRNA and $m R N A$ in human blood exosomes. Nucleic Acids Research, 2018. 46(D1): p. D106-D112.

160. Kanninen, K.M., et al., Exosomes as new diagnostic tools in CNS diseases. Biochimica et Biophysica Acta (BBA) - Molecular Basis of Disease, 2016. 1862(3): p. 403-410.

161. Robbins, P.D. and A.E. Morelli, Regulation of Immune Responses by Extracellular Vesicles. Nature reviews. Immunology, 2014. 14(3): p. 195-208.

162. Suchorska, W.M. and M.S. Lach, The role of exosomes in tumor progression and metastasis (Review). Oncol Rep, 2016. 35(3): p. 1237-44.

163. Ribeiro, M.F., et al., Exosomes Function in Pro- and Anti-Angiogenesis. Current angiogenesis, 2013. 2(1): p. 54-59. 
164. Setshedi, M., J.R. Wands, and S.M. de la Monte, Acetaldehyde adducts in alcoholic liver disease. Oxidative Medicine and Cellular Longevity, 2010. 3(3): p. 178-185.

165. Bradford, B.U., et al., Cytochrome P450 CYP2E1, but not nicotinamide adenine dinucleotide phosphate oxidase, is required for ethanol-induced oxidative DNA damage in rodent liver. Hepatology, 2005. 41(2): p. 336-44.

166. Cho, Y.E., et al., Exogenous exosomes from mice with acetaminophen-induced liver injury promote toxicity in the recipient hepatocytes and mice. Sci Rep, 2018. 8(1): p. 16070.

167. Basra, G., S. Basra, and S. Parupudi, Symptoms and signs of acute alcoholic hepatitis. World Journal of Hepatology, 2011. 3(5): p. 118-120.

168. Wang, H.J., et al., Inflammation in Alcoholic Liver Disease. Annual review of nutrition, 2012. 32: p. 343-368.

169. Celli, R. and X. Zhang, Pathology of Alcoholic Liver Disease. Journal of Clinical and Translational Hepatology, 2014. 2(2): p. 103-109.

170. Brandon-Warner, E., et al., Processing of miR17-92 Cluster in Hepatic Stellate Cells Promotes Hepatic Fibrogenesis During Alcohol-Induced Injury. Alcoholism, clinical and experimental research, 2016. 40(7): p. 1430-1442.

171. Bala, S., et al., Circulating microRNAs in exosomes indicate hepatocyte injury and inflammation in alcoholic, drug-induced, and inflammatory liver diseases. Hepatology, 2012. 56(5): p. 1946-57.

172. Viñas, J.L., et al., Receptor-Ligand Interaction Mediates Targeting of Endothelial Colony Forming Cell-derived Exosomes to the Kidney after Ischemic Injury. Scientific reports, 2018. 8(1): p. 16320-16320.

173. Qin, Y., et al., Myostatin inhibits osteoblastic differentiation by suppressing osteocyte-derived exosomal microRNA-218: A novel mechanism in muscle-bone communication. The Journal of biological chemistry, 2017. 292(26): p. 1102111033.

174. Hayes, C.N. and K. Chayama, MicroRNAs as Biomarkers for Liver Disease and Hepatocellular Carcinoma. International Journal of Molecular Sciences, 2016. 17(3): p. 280.

175. Momen-Heravi, F., et al., Increased number of circulating exosomes and their microRNA cargos are potential novel biomarkers in alcoholic hepatitis. J Transl Med, 2015. 13: p. 261.

176. Singal, A.K., et al., ACG Clinical Guideline: Alcoholic Liver Disease. Am J Gastroenterol, 2018. 113(2): p. 175-194.

177. Verma, V.K., et al., Alcohol stimulates macrophage activation through caspasedependent hepatocyte derived release of CD40L containing extracellular vesicles. J Hepatol, 2016. 64(3): p. 651-60.

178. Saha, B., et al., Extracellular vesicles from mice with alcoholic liver disease carry a distinct protein cargo and induce macrophage activation through heat shock protein 90. Hepatology, 2018. 67(5): p. 1986-2000.

179. Byrd, C.A., et al., Heat shock protein 90 mediates macrophage activation by Taxol and bacterial lipopolysaccharide. Proc Natl Acad Sci U S A, 1999. 96(10): p. 5645-50. 
180. Deshmane, S.L., et al., Monocyte Chemoattractant Protein-1 (MCP-1): An Overview. Journal of Interferon \& Cytokine Research, 2009. 29(6): p. 313-326.

181. Nojima, H., et al., Chemokine Receptors, CXCR1 and CXCR2, Differentially Regulate Exosome Release in Hepatocytes. PLoS One, 2016. 11(8): p. e0161443.

182. An, S., Y. Zheng, and T. Bleu, Sphingosine 1-phosphate-induced cell proliferation, survival, and related signaling events mediated by $G$ proteincoupled receptors Edg3 and Edg5. J Biol Chem, 2000. 275(1): p. 288-96.

183. Breitkopf, K., et al., Anti-TGF-beta strategies for the treatment of chronic liver disease. Alcohol Clin Exp Res, 2005. 29(11 Suppl): p. 121s-131s.

184. Brandon-Warner, E., et al., Processing of miR17-92 Cluster in Hepatic Stellate Cells Promotes Hepatic Fibrogenesis During Alcohol-Induced Injury. Alcohol Clin Exp Res, 2016. 40(7): p. 1430-42.

185. Saha, B., et al., MicroRNA Cargo of Extracellular Vesicles from Alcohol-exposed Monocytes Signals Naive Monocytes to Differentiate into M2 Macrophages. J Biol Chem, 2016. 291(1): p. 149-59.

186. Crews, F.T., et al., Cytokines and alcohol. Alcohol Clin Exp Res, 2006. 30(4): p. 720-30.

187. Kodidela, S., et al., Cytokine profiling of exosomes derived from the plasma of $H I V$-infected alcohol drinkers and cigarette smokers. PLoS One, 2018. 13(7): p. e0201144.

188. Lamichhane, T.N., et al., Ethanol Induces Enhanced Vascularization Bioactivity of Endothelial Cell-Derived Extracellular Vesicles via Regulation of MicroRNAs and Long Non-Coding RNAs. Sci Rep, 2017. 7(1): p. 13794.

189. Atienzar-Aroca, S., et al., Oxidative stress in retinal pigment epithelium cells increases exosome secretion and promotes angiogenesis in endothelial cells. J Cell Mol Med, 2016. 20(8): p. 1457-66.

190. Momen-Heravi, F. and S. Bala, Extracellular vesicles in oral squamous carcinoma carry oncogenic miRNA profile and reprogram monocytes via NFkappaB pathway. Oncotarget, 2018. 9(78): p. 34838-34854.

191. Malik, Z.A., et al., Cardiac myocyte exosomes: stability, HSP60, and proteomics. Am J Physiol Heart Circ Physiol, 2013. 304(7): p. H954-65.

192. Lazarevic, A.M., et al., Early changes in left ventricular function in chronic asymptomatic alcoholics: relation to the duration of heavy drinking. J Am Coll Cardiol, 2000. 35(6): p. 1599-606.

193. Charrier, A.L. and D.R. Brigstock, Connective tissue growth factor production by activated pancreatic stellate cells in mouse alcoholic chronic pancreatitis. Lab Invest, 2010. 90(8): p. 1179-88.

194. Charrier, A., et al., Connective tissue growth factor (CCN2) and microRNA-21 are components of a positive feedback loop in pancreatic stellate cells (PSC) during chronic pancreatitis and are exported in PSC-derived exosomes. J Cell Commun Signal, 2014. 8(2): p. 147-56.

195. Cho, Y.E., et al., Increased ethanol-inducible cytochrome P450-2E1 and cytochrome $P 450$ isoforms in exosomes of alcohol-exposed rodents and patients with alcoholism through oxidative and endoplasmic reticulum stress. Hepatol Commun, 2017. 1(7): p. 675-690. 
196. Brown, G.C. and A. Vilalta, How microglia kill neurons. Brain Res, 2015. 1628(Pt B): p. 288-297.

197. Amritraj, A., et al., Role of cathepsin D in U18666A-induced neuronal cell death: potential implication in Niemann-Pick type $C$ disease pathogenesis. J Biol Chem, 2013. 288(5): p. 3136-52.

198. Fan, Y. and J.J. He, HIV-1 Tat Promotes Lysosomal Exocytosis in Astrocytes and Contributes to Astrocyte-mediated Tat Neurotoxicity. J Biol Chem, 2016. 291(43): p. 22830-22840.

199. Hook, G., et al., Brain pyroglutamate amyloid-beta is produced by cathepsin B and is reduced by the cysteine protease inhibitor E64d, representing a potential Alzheimer's disease therapeutic. J Alzheimers Dis, 2014. 41(1): p. 129-49.

200. Higaki, J., et al., Processing of beta-amyloid precursor protein by cathepsin D. J Biol Chem, 1996. 271(50): p. 31885-93.

201. Wu, Z., et al., Cathepsin B plays a critical role in inducing Alzheimer's diseaselike phenotypes following chronic systemic exposure to lipopolysaccharide from Porphyromonas gingivalis in mice. Brain Behav Immun, 2017. 65: p. 350-361.

202. Snir, J.A., et al., An Aspartyl Cathepsin Targeted PET Agent: Application in an Alzheimer's Disease Mouse Model. J Alzheimers Dis, 2018. 61(3): p. 1241-1252.

203. Cataldo, A.M., et al., Gene expression and cellular content of cathepsin D in Alzheimer's disease brain: evidence for early up-regulation of the endosomallysosomal system. Neuron, 1995. 14(3): p. 671-80.

204. Di Domenico, F., A. Tramutola, and M. Perluigi, Cathepsin D as a therapeutic target in Alzheimer's disease. Expert Opin Ther Targets, 2016. 20(12): p. 13931395.

205. Embury, C.M., et al., Cathepsin B Improves ss-Amyloidosis and Learning and Memory in Models of Alzheimer's Disease. J Neuroimmune Pharmacol, 2017. 12(2): p. 340-352.

206. Hook, V., G. Hook, and M. Kindy, Pharmacogenetic features of cathepsin B inhibitors that improve memory deficit and reduce beta-amyloid related to Alzheimer's disease. Biol Chem, 2010. 391(8): p. 861-72.

207. Morena, F., et al., A Comparison of Lysosomal Enzymes Expression Levels in Peripheral Blood of Mild-and Severe-Alzheimer's Disease and MCI Patients: Implications for Regenerative Medicine Approaches. Int J Mol Sci, 2017. 18(8).

208. Kingham, P.J. and J.M. Pocock, Microglial secreted cathepsin B induces neuronal apoptosis. J Neurochem, 2001. 76(5): p. 1475-84.

209. Zenon, F., et al., HIV-infected microglia mediate cathepsin B-induced neurotoxicity. J Neurovirol, 2015. 21(5): p. 544-58.

210. Donohue, T.M., et al., Lysosomal leakage and lack of adaptation of hepatoprotective enzyme contribute to enhanced susceptibility to ethanol-induced liver injury in female rats. Alcohol Clin Exp Res, 2007. 31(11): p. 1944-52.

211. Amer, S.M., et al., Hepatic alcohol dehydrogenase deficiency induces pancreatic injury in chronic ethanol feeding model of deer mice. Exp Mol Pathol, 2018. 104(1): p. 89-97.

212. Bonner, A.B., et al., Lysosomal and nonlysosomal protease activities of the brain in response to ethanol feeding. Alcohol, 1995. 12(6): p. 505-9. 
213. Downs, C.A., et al., Hydrogen Peroxide Stimulates Exosomal Cathepsin B

Regulation of the Receptor for Advanced Glycation End-Products (RAGE). J Cell Biochem, 2018. 119(1): p. 599-606.

214. Castino, R., et al., Cathepsin D-Bax death pathway in oxidative stressed neuroblastoma cells. Free Radic Biol Med, 2007. 42(9): p. 1305-16.

215. Goetzl, E.J., et al., Altered lysosomal proteins in neural-derived plasma exosomes in preclinical Alzheimer disease. Neurology, 2015. 85(1): p. 40-7.

216. Kang, G.Y., et al., Exosomal proteins in the aqueous humor as novel biomarkers in patients with neovascular age-related macular degeneration. J Proteome Res, 2014. 13(2): p. 581-95.

217. Segal, A.W., The function of the NADPH oxidase of phagocytes and its relationship to other NOXs in plants, invertebrates, and mammals. Int J Biochem Cell Biol, 2008. 40(4): p. 604-18.

218. Qin, L. and F.T. Crews, NADPH oxidase and reactive oxygen species contribute to alcohol-induced microglial activation and neurodegeneration. $\mathrm{J}$ Neuroinflammation, 2012. 9: p. 5.

219. Wang, X., et al., Cdc42-dependent activation of NADPH oxidase is involved in ethanol-induced neuronal oxidative stress. PLoS One, 2012. 7(5): p. e38075.

220. Janiszewski, M., et al., Platelet-derived exosomes of septic individuals possess proapoptotic $N A D(P) H$ oxidase activity: A novel vascular redox pathway. Crit Care Med, 2004. 32(3): p. 818-25.

221. Huang, W.J., X. Zhang, and W.W. Chen, Association between alcohol and Alzheimer's disease. Exp Ther Med, 2016. 12(3): p. 1247-1250.

222. Wang, X., et al., Oxidative stress and mitochondrial dysfunction in Alzheimer's disease. Biochim Biophys Acta, 2014. 1842(8): p. 1240-7.

223. Simic, G., et al., Tau Protein Hyperphosphorylation and Aggregation in Alzheimer's Disease and Other Tauopathies, and Possible Neuroprotective Strategies. Biomolecules, 2016. 6(1): p. 6.

224. Saito, M., et al., Tau phosphorylation and cleavage in ethanol-induced neurodegeneration in the developing mouse brain. Neurochem Res, 2010. 35(4): p. 651-9.

225. Gendron, T.F., et al., Ethanol enhances tau accumulation in neuroblastoma cells that inducibly express tau. Neurosci Lett, 2008. 443(2): p. 67-71.

226. Saman, S., et al., Exosome-associated tau is secreted in tauopathy models and is selectively phosphorylated in cerebrospinal fluid in early Alzheimer disease. J Biol Chem, 2012. 287(6): p. 3842-9.

227. Asai, H., et al., Depletion of microglia and inhibition of exosome synthesis halt tau propagation. Nat Neurosci, 2015. 18(11): p. 1584-93.

228. Miranda, R.C., et al., MicroRNAs: master regulators of ethanol abuse and toxicity? Alcohol Clin Exp Res, 2010. 34(4): p. 575-87.

229. Dippold, R.P., et al., Chronic ethanol feeding enhances miR-21 induction during liver regeneration while inhibiting proliferation in rats. Am J Physiol Gastrointest Liver Physiol, 2012. 303(6): p. G733-43.

230. Juskeviciute, E., et al., Inhibition of miR-21 rescues liver regeneration after partial hepatectomy in ethanol-fed rats. Am J Physiol Gastrointest Liver Physiol, 2016. 311(5): p. G794-g806. 
231. Ge, X.T., et al., miR-21 improves the neurological outcome after traumatic brain injury in rats. Sci Rep, 2014. 4: p. 6718.

232. Chan, J.A., A.M. Krichevsky, and K.S. Kosik, MicroRNA-21 is an antiapoptotic factor in human glioblastoma cells. Cancer Res, 2005. 65(14): p. 6029-33.

233. Santangelo, A., et al., A microRNA signature from serum exosomes of patients with glioma as complementary diagnostic biomarker. J Neurooncol, 2018. 136(1): p. 51-62.

234. Shi, R., et al., Exosomal levels of miRNA-21 from cerebrospinal fluids associated with poor prognosis and tumor recurrence of glioma patients. Oncotarget, 2015. 6(29): p. 26971-81.

235. Yelamanchili, S.V., et al., MiR-21 in Extracellular Vesicles Leads to Neurotoxicity via TLR7 Signaling in SIV Neurological Disease. PLoS Pathog, 2015. 11(7): p. e1005032.

236. Kumar, S., et al., Alcohol consumption effect on antiretroviral therapy and HIV-1 pathogenesis: role of cytochrome P450 isozymes. Expert Opin Drug Metab Toxicol, 2012. 8(11): p. 1363-75.

237. Ande, A., et al., Enhanced oxidative stress by alcohol use in HIV+ patients: possible involvement of cytochrome P450 2E1 and antioxidant enzymes. AIDS Res Ther, 2015. 12: p. 29.

238. Rahman, M.A., Y. Gong, and S. Kumar, In vitro evaluation of structural analogs of diallyl sulfide as novel CYP2E1 inhibitors for their protective effect against xenobiotic-induced toxicity and HIV replication. Toxicol Lett, 2018. 292: p. 3138.

239. Gendron, K., et al., The activity of the HIV-1 IRES is stimulated by oxidative stress and controlled by a negative regulatory element. Nucleic Acids Res, 2011. 39(3): p. 902-12.

240. Ivanov, A.V., et al., Oxidative Stress during HIV Infection: Mechanisms and Consequences. Oxid Med Cell Longev, 2016. 2016: p. 8910396.

241. Dong, Q., et al., Ethanol enhances TNF-alpha-inducible NFkappaB activation and HIV-1-LTR transcription in CD4+ Jurkat T lymphocytes. J Lab Clin Med, 2000. 136(5): p. 333-43.

242. Silverstein, P.S. and A. Kumar, HIV-1 and alcohol: interactions in the central nervous system. Alcohol Clin Exp Res, 2014. 38(3): p. 604-10.

243. Fama, R., et al., Working and episodic memory in HIV infection, alcoholism, and their comorbidity: baseline and 1-year follow-up examinations. Alcohol Clin Exp Res, 2009. 33(10): p. 1815-24.

244. Midde, N.M., et al., Alterations in cellular pharmacokinetics and pharmacodynamics of elvitegravir in response to ethanol exposure in HIV-1 infected monocytic (U1) cells. PLoS One, 2017. 12(2): p. e0172628.

245. Midde, N.M., et al., Effect of Ethanol on the Metabolic Characteristics of HIV-1 Integrase Inhibitor Elvitegravir and Elvitegravir/Cobicistat with CYP3A: An Analysis Using a Newly Developed LC-MS/MS Method. PLoS One, 2016. 11(2): p. e0149225.

246. Self, R.L., et al., Cytotoxic effects of exposure to the human immunodeficiency virus type 1 protein Tat in the hippocampus are enhanced by prior ethanol treatment. Alcohol Clin Exp Res, 2004. 28(12): p. 1916-24. 
247. Chen, W., et al., Ethanol potentiates HIV-1 gp120-induced apoptosis in human neurons via both the death receptor and NMDA receptor pathways. Virology, 2005. 334(1): p. 59-73.

248. Sampey, G.C., et al., Exosomes and their role in CNS viral infections. J Neurovirol, 2014. 20(3): p. 199-208.

249. Madison, M.N. and C.M. Okeoma, Exosomes: Implications in HIV-1 Pathogenesis. Viruses, 2015. 7(7): p. 4093-118.

250. Anderson, M., F. Kashanchi, and S. Jacobson, Role of Exosomes in Human Retroviral Mediated Disorders. J Neuroimmune Pharmacol, 2018. 13(3): p. 279291.

251. Guo, W.J., et al., Gender-specific interactions between alcohol metabolism genes and severity of quantitative alcohol-related-traits in a Tibetan population. Neurosci Lett, 2011. 495(1): p. 22-5.

252. Patters, B., The Role of Exosomal Transport of Viral Agents in Persistent HIV Pathogenesis. 2018.

253. Cho, Y.E., et al., Extracellular vesicles as potential biomarkers for alcohol- and drug-induced liver injury and their therapeutic applications. Pharmacol Ther, 2018.

254. Gold, B., et al., Do circulating tumor cells, exosomes, and circulating tumor nucleic acids have clinical utility? A report of the association for molecular pathology. J Mol Diagn, 2015. 17(3): p. 209-24.

255. Revenfeld, A.L., et al., Diagnostic and prognostic potential of extracellular vesicles in peripheral blood. Clin Ther, 2014. 36(6): p. 830-46.

256. Hamlett, E.D., et al., Neuronal exosomes reveal Alzheimer's disease biomarkers in Down syndrome. Alzheimers Dement, 2017. 13(5): p. 541-549.

257. Visani, M., et al., Expression of 19 microRNAs in glioblastoma and comparison with other brain neoplasia of grades I-III. Mol Oncol, 2014. 8(2): p. 417-30.

258. Lee, S.S., et al., Role of CYP2E1 in the hepatotoxicity of acetaminophen. J Biol Chem, 1996. 271(20): p. 12063-7.

259. Lu, Y. and A.I. Cederbaum, CYP2E1 and oxidative liver injury by alcohol. Free Radic Biol Med, 2008. 44(5): p. 723-38.

260. French, S.W., The importance of CYP2E1 in the pathogenesis of alcoholic liver disease and drug toxicity and the role of the proteasome. Subcell Biochem, 2013. 67: p. 145-64.

261. Kessova, I. and A.I. Cederbaum, CYP2E1: biochemistry, toxicology, regulation and function in ethanol-induced liver injury. Curr Mol Med, 2003. 3(6): p. 50918.

262. Wang, T., et al., Quantitative structure-activity relationship: promising advances in drug discovery platforms. Expert Opin Drug Discov, 2015. 10(12): p. 1283300.

263. Sharma, S., et al., Design strategies, structure activity relationship and mechanistic insights for purines as kinase inhibitors. Eur J Med Chem, 2016. 112: p. 298-346.

264. Ferreira, L.G., et al., Molecular docking and structure-based drug design strategies. Molecules, 2015. 20(7): p. 13384-421. 
265. Lohning, A.E., et al., A Practical Guide to Molecular Docking and Homology Modelling for Medicinal Chemists. Curr Top Med Chem, 2017. 17(18): p. 20232040.

266. Lin, J., et al., Exosomes: novel biomarkers for clinical diagnosis. ScientificWorldJournal, 2015. 2015: p. 657086.

267. Desdin-Mico, G. and M. Mittelbrunn, Role of exosomes in the protection of cellular homeostasis. Cell Adh Migr, 2017. 11(2): p. 127-134.

268. Emanueli, C., et al., Exosomes and exosomal miRNAs in cardiovascular protection and repair. Vascul Pharmacol, 2015. 71: p. 24-30.

269. Record, M., et al., Exosomes as new vesicular lipid transporters involved in cellcell communication and various pathophysiologies. Biochim Biophys Acta, 2014. 1841(1): p. 108-20.

270. Ghanem, C.I., et al., Acetaminophen from liver to brain: New insights into drug pharmacological action and toxicity. Pharmacol Res, 2016. 109: p. 119-31.

271. Yoon, E., et al., Acetaminophen-Induced Hepatotoxicity: a Comprehensive Update. J Clin Transl Hepatol, 2016. 4(2): p. 131-42.

272. Jin, M., et al., Regulation of cytochrome P450 2el expression by ethanol: role of oxidative stress-mediated pkc/jnk/sp1 pathway. Cell Death \& Disease, 2013. 4(3): p. e554.

273. Raza, H. and A. John, Differential Cytotoxicity of Acetaminophen in Mouse Macrophage J774.2 and Human Hepatoma HepG2 Cells: Protection by Diallyl Sulfide. PLoS One, 2015. 10(12): p. e0145965.

274. Zhang, R.H., et al., Inhibition of CYP2E1 attenuates chronic alcohol intakeinduced myocardial contractile dysfunction and apoptosis. Biochim Biophys Acta, 2013. 1832(1): p. 128-41.

275. Swaminathan, K., D.L. Clemens, and A. Dey, Inhibition of CYP2E1 leads to decreased malondialdehyde-acetaldehyde adduct formation in VL-17A cells under chronic alcohol exposure. Life Sci, 2013. 92(6-7): p. 325-36.

276. Jin, M., A. Kumar, and S. Kumar, Ethanol-mediated regulation of cytochrome P450 2A6 expression in monocytes: role of oxidative stress-mediated PKC/MEK/Nrf2 pathway. PLoS One, 2012. 7(4): p. e35505.

277. Chen, J., et al., Discovery of novel 2-aryl-4-benzoyl-imidazole (ABI-III) analogues targeting tubulin polymerization as antiproliferative agents. J Med Chem, 2012. 55(16): p. 7285-9.

278. Lu, Y., et al., Design, Synthesis, and Biological Evaluation of Stable Colchicine Binding Site Tubulin Inhibitors as Potential Anticancer Agents. Journal of Medicinal Chemistry, 2014. 57(17): p. 7355-7366.

279. Porubsky, P.R., K.P. Battaile, and E.E. Scott, Human cytochrome P450 2E1 structures with fatty acid analogs reveal a previously unobserved binding mode. J Biol Chem, 2010. 285(29): p. 22282-90.

280. Cederbaum, A.I., Methodology to assay CYP2E1 mixed function oxidase catalytic activity and its induction. Redox Biology, 2014. 2: p. 1048-1054.

281. Collom, S.L., et al., CYP2E1 Substrate Inhibition: MECHANISTIC INTERPRETATION THROUGH AN EFFECTOR SITE FOR MONOCYCLIC COMPOUNDS(). J Biol Chem, 2008. 283(6): p. 3487-96. 
282. Ramirez, D. and J. Caballero, Is It Reliable to Use Common Molecular Docking Methods for Comparing the Binding Affinities of Enantiomer Pairs for Their Protein Target? Int J Mol Sci, 2016. 17(4).

283. Kitchen, D.B., et al., Docking and scoring in virtual screening for drug discovery: methods and applications. Nat Rev Drug Discov, 2004. 3(11): p. 935-49.

284. Morris, C.R., et al., Inhibition by allyl sulfides and phenethyl isothiocyanate of methyl-n-pentylnitrosamine depentylation by rat esophageal microsomes, human and rat CYP2E1, and Rat CYP2A3. Nutr Cancer, 2004. 48(1): p. 54-63.

285. Zhai, Q., et al., Oxidative stress potentiated by diallylsulfide, a selective CYP2E1 inhibitor, in isoniazid toxic effect on rat primary hepatocytes. Toxicol Lett, 2008. 183(1-3): p. 95-8.

286. Lieber, C.S., Cytochrome P-4502E1: its physiological and pathological role. Physiol Rev, 1997. 77(2): p. 517-44.

287. Rao, P.S. and S. Kumar, Chronic Effects of Ethanol and/or Darunavir/Ritonavir on U937 Monocytic Cells: Regulation of Cytochrome P450 and Antioxidant Enzymes, Oxidative Stress, and Cytotoxicity. Alcohol Clin Exp Res, 2016. 40(1): p. 73-82.

288. Fontana, R.J., Acute Liver Failure including Acetaminophen Overdose. The Medical clinics of North America, 2008. 92(4): p. 761-794.

289. Rahman, M.A., et al., Kinetic characterizations of diallyl sulfide analogs for their novel role as CYP2E1 enzyme inhibitors. Pharmacology Research \& Perspectives, 2017. 5(5): p. e00362.

290. Rao, P.S.S. and S. Kumar, Chronic effects of ethanol and/or darunavir/ritonavir on U937 monocytic cells: Regulation of cytochrome P450 and antioxidant enzymes, oxidative stress, and cytotoxicity. Alcoholism, clinical and experimental research, 2016. 40(1): p. 73-82.

291. Major, E.O., et al., Establishment of a line of human fetal glial cells that supports JC virus multiplication. Proc Natl Acad Sci U S A, 1985. 82(4): p. 1257-61.

292. Brady, J.F., et al., Inhibition of cytochrome P-450 2E1 by diallyl sulfide and its metabolites. Chemical Research in Toxicology, 1991. 4(6): p. 642-647.

293. Collom, S.L., et al., CYP2E1 Active Site Residues in Substrate Recognition Sequence 5 Identified by Photoaffinity Labeling and Homology Modeling. Arch Biochem Biophys, 2007. 459(1): p. 59-69.

294. Rahman, M.A., Gong, Y., Kumar, S., Kinetic characterizations of diallyl sulfide analogs for their novel role as CYP2E1 enzyme inhibitors. Pharmacology Research \& Perspectives 2017.

295. Shah, A., et al., HIV gp120- and methamphetamine-mediated oxidative stress induces astrocyte apoptosis via cytochrome P450 2E1. Cell Death \& Disease, 2013. 4(10): p. e850.

296. Wu, P.P., et al., Diallyl sulfide induces cell cycle arrest and apoptosis in HeLa human cervical cancer cells through the p53, caspase- and mitochondriadependent pathways. Int J Oncol, 2011. 38(6): p. 1605-13.

297. Holmuhamedov, E.L., et al., Ethanol suppresses ureagenesis in rat hepatocytes: role of acetaldehyde. J Biol Chem, 2012. 287(10): p. 7692-700.

298. Louvet, A. and P. Mathurin, Alcoholic liver disease: mechanisms of injury and targeted treatment. Nat Rev Gastroenterol Hepatol, 2015. 12(4): p. 231-42. 
299. Singal, A.K., et al., Alcoholic hepatitis: current challenges and future directions. Clin Gastroenterol Hepatol, 2014. 12(4): p. 555-64; quiz e31-2.

300. Wu, D. and A.I. Cederbaum, Oxidative stress mediated toxicity exerted by ethanol-inducible CYP2E1. Toxicol Appl Pharmacol, 2005. 207(2 Suppl): p. 70-6.

301. Ko, J.W., et al., Protective effects of diallyl disulfide against acetaminopheninduced nephrotoxicity: A possible role of CYP2E1 and NF-kappaB. Food Chem Toxicol, 2017. 102: p. 156-165.

302. Cheung, C., et al., The cyp2e1-humanized transgenic mouse: role of cyp2el in acetaminophen hepatotoxicity. Drug Metab Dispos, 2005. 33(3): p. 449-57.

303. Gong, Y., et al., The role of cytochrome P450 $2 E 1$ on ethanol-mediated oxidative stress and HIV replication in human monocyte-derived macrophages. Biochem Biophys Rep, 2019. 17: p. 65-70.

304. Conde-Vancells, J., et al., Characterization and comprehensive proteome profiling of exosomes secreted by hepatocytes. J Proteome Res, 2008. 7(12): p. 5157-66.

305. Isola, A.L. and S. Chen, Exosomes: The Messengers of Health and Disease. Curr Neuropharmacol, 2017. 15(1): p. 157-165.

306. Ferguson, S.W. and J. Nguyen, Exosomes as therapeutics: The implications of molecular composition and exosomal heterogeneity. J Control Release, 2016. 228: p. 179-190.

307. Hudry, E., et al., Exosome-associated AAV vector as a robust and convenient neuroscience tool. Gene Ther, 2016. 23(4): p. 380-92.

308. Haney, M.J., et al., Exosomes as drug delivery vehicles for Parkinson's disease therapy. J Control Release, 2015. 207: p. 18-30.

309. Ruivo, C.F., et al., The Biology of Cancer Exosomes: Insights and New Perspectives. Cancer Res, 2017. 77(23): p. 6480-6488.

310. Guay, C. and R. Regazzi, Exosomes as new players in metabolic organ cross-talk. Diabetes Obes Metab, 2017. 19 Suppl 1: p. 137-146.

311. Ye, W., et al., Plasma-derived exosomes contribute to inflammation via the TLR9NF-kappaB pathway in chronic heart failure patients. Mol Immunol, 2017. 87: p. 114-121.

312. Yu, X., M. Odenthal, and J.W. Fries, Exosomes as miRNA Carriers: FormationFunction-Future. Int J Mol Sci, 2016. 17(12).

313. Street, J.M., et al., Urine Exosomes: An Emerging Trove of Biomarkers. Adv Clin Chem, 2017. 78: p. 103-122.

314. Stuendl, A., et al., Induction of alpha-synuclein aggregate formation by CSF exosomes from patients with Parkinson's disease and dementia with Lewy bodies. Brain, 2016. 139(Pt 2): p. 481-94.

315. Wang, J., et al., Plasma exosomes as novel biomarker for the early diagnosis of gastric cancer. Cancer Biomark, 2018. 21(4): p. 805-812.

316. Whiteside, T.L., The emerging role of plasma exosomes in diagnosis, prognosis and therapies of patients with cancer. Contemp Oncol (Pozn), 2018. 22(1a): p. $38-40$.

317. Xie, J.X., et al., MicroRNA profiling in kidney disease: Plasma versus plasmaderived exosomes. Gene, 2017. 627: p. 1-8. 
318. Hannafon, B.N., et al., Plasma exosome microRNAs are indicative of breast cancer. Breast Cancer Res, 2016. 18(1): p. 90.

319. Dorayappan, K.D.P., et al., The biological significance and clinical applications of exosomes in ovarian cancer. Gynecol Oncol, 2016. 142(1): p. 199-205.

320. Zhang, W., et al., Extracellular vesicles in diagnosis and therapy of kidney diseases. Am J Physiol Renal Physiol, 2016. 311(5): p. F844-f851.

321. Kumar, S., et al., Specific packaging and circulation of cytochromes P450, especially $2 E 1$ isozyme, in human plasma exosomes and their implications in cellular communications. Biochemical and Biophysical Research Communications, 2017. 491(3): p. 675-680.

322. Kim, S.N., et al., Induction of hepatic CYP2E1 by a subtoxic dose of acetaminophen in rats: increase in dichloromethane metabolism and carboxyhemoglobin elevation. Drug Metab Dispos, 2007. 35(10): p. 1754-8.

323. Andersson, T.B., K.P. Kanebratt, and J.G. Kenna, The HepaRG cell line: a unique in vitro tool for understanding drug metabolism and toxicology in human. Expert Opin Drug Metab Toxicol, 2012. 8(7): p. 909-20.

324. Gong, Y., et al., Novel elvitegravir nanoformulation approach to suppress the viral load in HIV-infected macrophages. Biochem Biophys Rep, 2017. 12: p. 214219.

325. Haque, S., et al., Monocyte-derived exosomes upon exposure to cigarette smoke condensate alter their characteristics and show protective effect against cytotoxicity and HIV-1 replication. Sci Rep, 2017. 7(1): p. 16120.

326. Bucher, S., et al., Co-exposure to benzo[a]pyrene and ethanol induces a pathological progression of liver steatosis in vitro and in vivo. Scientific Reports, 2018. 8(1): p. 5963.

327. Do, T.H., et al., Iron-mediated effect of alcohol on hepatocyte differentiation in HepaRG cells. Chem Biol Interact, 2013. 206(2): p. 117-25.

328. Bucher, S., et al., Possible Involvement of Mitochondrial Dysfunction and Oxidative Stress in a Cellular Model of NAFLD Progression Induced by Benzo[a]pyrene/Ethanol CoExposure. Oxidative Medicine and Cellular Longevity, 2018. 2018: p. 18.

329. Rahman, M.A., Y. Gong, and S. Kumar, In vitro evaluation of structural analogs of diallyl sulfide as novel CYP2E1 inhibitors for their protective effect against xenobiotic-induced toxicity and HIV replication. Toxicology Letters, 2018. 292: p. 31-38.

330. Midde, N.M., et al., Effect of Ethanol on the Metabolic Characteristics of HIV-1 Integrase Inhibitor Elvitegravir and Elvitegravir/Cobicistat with CYP3A: An Analysis Using a Newly Developed LC-MS/MS Method. PloS one, 2016. 11(2): p. e0149225-e0149225.

331. Laine, J.E., et al., Acetaminophen bioactivation by human cytochrome P450 enzymes and animal microsomes. Xenobiotica, 2009. 39(1): p. 11-21.

332. He, X. and S. Feng, Role of Metabolic Enzymes P450 (CYP) on Activating Procarcinogen and their Polymorphisms on the Risk of Cancers. Curr Drug Metab, 2015. 16(10): p. 850-63. 
333. Ranjit, S., et al., Effect of Polyaryl Hydrocarbons on Cytotoxicity in Monocytic Cells: Potential Role of Cytochromes P450 and Oxidative Stress Pathways. PLoS One, 2016. 11(9): p. e 0163827.

334. Bernauer, U., et al., CYP2E1-dependent benzene toxicity: the role of extra-hepatic benzene metabolism. Arch Toxicol, 1999. 73(4-5): p. 189-96.

335. Garc, et al., The Role of CYP2E1 in the Drug Metabolism or Bioactivation in the Brain. Oxidative Medicine and Cellular Longevity, 2017. 2017: p. 14.

336. Gonzalez, F.J., Role of cytochromes P450 in chemical toxicity and oxidative stress: studies with CYP2E1. Mutat Res, 2005. 569(1-2): p. 101-10.

337. Colombo, M., G. Raposo, and C. Théry, Biogenesis, Secretion, and Intercellular Interactions of Exosomes and Other Extracellular Vesicles. Annual Review of Cell and Developmental Biology, 2014. 30(1): p. 255-289.

338. Ranjit, S., et al., Potential neuroprotective role of astroglial exosomes against smoking-induced oxidative stress and HIV-1 replication in the central nervous system. Expert Opin Ther Targets, 2018. 22(8): p. 703-714.

339. Fyfe, I., Exosomes can spread toxic AD pathology. Nature Reviews Neurology, 2018. 14(8): p. 451-451.

340. Cho, Y.-E., et al., Exogenous exosomes from mice with acetaminophen-induced liver injury promote toxicity in the recipient hepatocytes and mice. Scientific Reports, 2018. 8(1): p. 16070.

341. Momen-Heravi, F., et al., Exosomes derived from alcohol-treated hepatocytes horizontally transfer liver specific miRNA-122 and sensitize monocytes to LPS. Scientific Reports, 2015. 5: p. 9991.

342. Cho, Y.-E., et al., Exogenous exosomes from mice with acetaminophen-induced liver injury promote toxicity in the recipient hepatocytes and mice. Scientific reports, 2018. 8(1): p. 16070-16070.

343. Holman, N.S., et al., Subtoxic Alterations in Hepatocyte-Derived Exosomes: An Early Step in Drug-Induced Liver Injury? Toxicol Sci, 2016. 151(2): p. 365-75.

344. Hannafon, B.N., et al., Plasma exosome microRNAs are indicative of breast cancer. Breast Cancer Research, 2016. 18(1): p. 90.

345. Magenta, A., et al., miR-200c is upregulated by oxidative stress and induces endothelial cell apoptosis and senescence via ZEB1 inhibition. Cell Death Differ, 2011. 18(10): p. 1628-39.

346. Carlomosti, F., et al., Oxidative Stress-Induced miR-200c Disrupts the Regulatory Loop Among SIRT1, FOXO1, and eNOS. Antioxid Redox Signal, 2017. 27(6): p. 328-344.

347. Bu, H., et al., MicroRNA Regulation of Oxidative Stress-Induced Cellular Senescence. Oxidative Medicine and Cellular Longevity, 2017. 2017: p. 12.

348. Magenta, A., et al., Oxidative stress and microRNAs in vascular diseases. Int J Mol Sci, 2013. 14(9): p. 17319-46.

349. Rodrigues, D. and A. Rowland, From Endogenous Compounds as Biomarkers to Plasma-Derived Nanovesicles as Liquid Biopsy; Has the Golden Age of Translational PK-ADME-DDI Science Finally Arrived? Clinical Pharmacology \& Therapeutics, 2018. 0(ja). 
350. Anzenbacher, P. and E. Anzenbacherová, Cytochromes P450 and metabolism of xenobiotics. Cellular and Molecular Life Sciences CMLS, 2001. 58(5): p. 737747. 


\section{VITA}

Mohammad Arifur Rahman, son of Mr. Ishaque Ali and Mrs. Hosne Ara, was born in 1988 in a small village of Satkhira district in Bangladesh. After finishing his elementary, middle, and high school education from the local institutions, he moved to the capital city, Dhaka, to attend University of Dhaka for his undergraduate degree in Pharmacy. After completion, he joined Incepta Pharmaceuticals Limited as a Product Executive which he continued for 3 years. During this period, he also completed his master's degree in Clinical Pharmacy and Pharmacology from University of Dhaka. Due to his keen interest in drug metabolism and toxicology research, he moved to the United States in 2014, to attend the University of Tennessee Health Science Center, Memphis. He joined Dr. Santosh Kumar's lab in the College of Pharmacy to pursue his doctoral degree in Pharmaceutical Sciences. He will be graduating in June, 2019. 